NORTHWESTERN UNIVERSITY

\title{
The Higgs Boson as a Window to Beyond the Standard Model
}

\author{
A DISSERTATION \\ SUBMITTED TO THE GRADUATE SCHOOL \\ IN PARTIAL FULFILLMENT OF THE REQUIREMENTS \\ for the degree \\ DOCTOR OF PHILOSOPHY
}

Field of Theoretical Physics

By

Roberto Vega-Morales

EVANSTON, ILLINOIS

August 2013 


\section{ABSTRACT \\ The Higgs Boson as a Window to Beyond the Standard Model}

\section{Roberto Vega-Morales}

In this thesis we examine the Higgs boson and its possible connections to physics beyond the standard model. In particular, we study the Higgs couplings to pairs of neutral electroweak gauge bosons in the charged lepton final state known as the 'golden channel'. We assess the ability of this channel to distinguish between various hypotheses for the nature of the newly discovered scalar particle. We also explore an explicit extension of the standard model which can explain various puzzles which remain unanswered even after the discovery of the Higgs boson. Specifically this model explains the existence of neutrino masses as well as a stable dark matter candidate and the non-observation of processes which violate overall lepton number. We examine in detail the implications of this model on Higgs phenomenology at the LHC as well as dark matter detection experiments. 


\section{Acknowledgements}

I would first like to acknowledge my various 'advisors' starting with Tim Tait for taking a chance on two clueless graduate students in myself and Kunal Kumar when he had no evidence of any reason for doing so. I would like to thank Ian Low for staying patient while I tried to figure out what was going on in our studies of the Higgs. I believe that time spent struggling is what ultimately led to my involvement with the CMS experiment as well as my time at Fermilab and I am very grateful. Thank you to Joe Lykken for sponsoring and advising me as part of the Fermilab Graduate Student Fellowship. I would like to thank André de Gouvêa for the many times he let me come into his office and bug him with silly questions. I must thank Kunal Kumar for being 'my partner in crime' as we spent countless hours discussing and chasing our tails trying to learn particle physics. I would also like to thank all my friends and roommates throughout my time in graduate school for putting up with my absence and lameness on so many occasions. I thank my dad for trying to talk me out of doing particle physics for so many years which of course meant that is exactly what I would do and also for supporting me when I inevitably followed in his footsteps. To my mom for her unconditional support even when I had no idea what I was doing and for making me feel like no matter what she would love it. Most of all, I am grateful to my wife Amanda for putting up with a theoretical particle physics graduate student on a daily basis while all the while making the time spent wonderful. This could not have happened without you. 


\section{Dedication}

Para mi Chuleta 


\section{Table of Contents}

\begin{tabular}{ll}
\hline ABSTRACT & 2
\end{tabular}

\begin{tabular}{|l|l|}
\hline Acknowledgements & 3 \\
\hline
\end{tabular}

$\begin{array}{ll}\text { Dedication } & 4\end{array}$

\begin{tabular}{ll}
\hline List of Figures & 7 \\
\hline
\end{tabular}

$\begin{array}{lll}\text { Chapter 1. Introduction } & 10\end{array}$

1.1. The Standard Model and the Need For New Physics 10

\begin{tabular}{lll}
\hline 1.2. & The Higgs as a Probe of New Physics & 12
\end{tabular}

Chapter 2. Scrutinizing the Higgs Signal and Background in the $2 e 2 \mu$ $\begin{array}{ll}\text { Golden Channel } & 15\end{array}$

$\begin{array}{lll}2.1 . & \text { Introduction } & 15\end{array}$

$\begin{array}{lll}2.2 . & \text { Four Lepton Events } & 18\end{array}$

$\begin{array}{lll}2.3 . & \text { Signal } & 20\end{array}$

$\begin{array}{lll}2.4 . & \text { Background } & 29\end{array}$

2.5. Conclusions and Outlook 43

Chapter 3. Directly Measuring the Tensor Structure of the Scalar Coupling $\begin{array}{ll}\text { to Gauge Bosons } & 47\end{array}$

3.1. Differential Spectrums 49 
3.2. $\quad$ Distinguishing Operators 54

3.3. Conclusions and Outlook 56

Chapter 4. Dark Matter and Vector-like Leptons From Gauged Lepton $\begin{array}{ll}\text { Number } & 59\end{array}$

4.1. Introduction 59

$\begin{array}{lll}\text { 4.2. The Model } & 61\end{array}$

\begin{tabular}{lll}
\hline 4.3. Dark Matter & 71
\end{tabular}

4.4. LHC Phenomenology and Constraints 81

4.5. Conclusions/Outlook 94

\begin{tabular}{ll}
\hline References & 97
\end{tabular}

\begin{tabular}{lll}
\hline Appendix A. Appendix & 103
\end{tabular}

\begin{tabular}{lll}
\hline A.1. Angular Distributions & 103
\end{tabular} 


\section{List of Figures}

$2.1 \quad$ Definition of angles in the four lepton CM frame. 20

$2.2 \quad$ Feynman diagrams contributing to $\varphi \rightarrow 2 \ell_{1} 2 \ell_{2}$. 23

$2.3 \quad$ Golden channel scalar differential mass spectrums. 29

$2.4 \quad$ Feynman diagrams contributing to $q \bar{q} \rightarrow Z Z \rightarrow 2 e 2 \mu$ and

$q \bar{q} \rightarrow Z \rightarrow 2 e 2 \mu$.

$2.5 \quad$ Feynman diagrams contributing to $q \bar{q} \rightarrow V_{i} V_{j} \rightarrow 2 \ell_{1} 2 \ell_{2}$ and

$q \bar{q} \rightarrow V_{i} \rightarrow 2 \ell_{1} 2 \ell_{2}$.

$2.6 \quad$ The four lepton system invariant mass spectrum (without $p d f s$ ) for the

$\begin{array}{ll}\text { various background components. } & 41\end{array}$

$2.7 \quad$ Comparing the LO and NLO results for the $M_{1}$ and $M_{2}$ invariant mass

$\begin{array}{ll}\text { spectra } & 43\end{array}$

$2.8 \quad$ Comparing the LO and NLO results for the polar angles

$\begin{array}{ll}\cos \Theta, \cos \theta_{1}, \cos \theta_{2} . & 44\end{array}$

$2.9 \quad$ Comparing the LO and NLO results for the azimuthal angles $\Phi$ and

$\Phi_{1}$.

$3.1 \quad$ Normalized distributions for $\Phi$ (top), $\cos \theta_{i}$ (middle), and $M_{2} . \quad 50$

$\begin{array}{lll}3.2 & \text { Normalized distribution for } \cos \theta \text {. } & 53\end{array}$ 
$3.3 \quad$ Normalized $M_{2}$ distributions.

$3.4 \quad$ Normalized distribution of our test statistic $\Lambda$. 55

$3.5 \quad$ Expected significance as a function of number of events. $\quad 57$

$4.1 \quad$ Contours of Higgs mixing angle $\theta$ (red-dotted), Higgs quartic coupling $\lambda_{H}$ (orange-solid), and heavy scalar mass $m_{\phi}$ in $\mathrm{GeV}$.

$4.2 \quad$ Contours of the $Z-Z_{L}$ mixing angle. $\quad 69$

$4.3 \quad$ Diagrams leading to $s$-channel $\nu_{X} \bar{\nu}_{X}$ annihilation into SM states. $\quad 74$

$4.4 \quad$ Relic density as a function of DM mass and vev $v_{\phi}$.

$4.5 \quad$ Diagrams leading to scattering with nucleons. 78

4.6 DM-nucleon cross section in pb, as a function of the Higgs mixing

$\begin{array}{ll}\text { angle. } & 79\end{array}$

4.7 DM-nucleon cross section in pb, as a function of the DM-Z coupling. 80

$4.8 \quad$ Fits to the full Higgs data set. 88

$4.9 \quad$ Contours of relative diphoton rate 1.

$4.10 \quad$ Contours of relative diphoton rate $2 . \quad 90$

$4.11 \quad$ Contours of relative diphoton rate 3.

$4.12 \quad$ Cross section for the process $p p \rightarrow \ell^{+} \ell^{-} Z_{L}$ at the $14 \mathrm{TeV}$ LHC. $\quad 92$

$4.13 \quad$ Cross sections for the pair production of exotic leptons at the $14 \mathrm{TeV}$

$\begin{array}{ll}\text { LHC. } & 92\end{array}$

\begin{tabular}{lll}
\hline A.1 Signal and Background $\cos \Theta$ angular distributions. & 107
\end{tabular} 
\begin{tabular}{lll}
\hline A.2 Signal and Background $\cos \theta_{1}$ angular distributions. & 107
\end{tabular}

\begin{tabular}{lll}
\hline A.3 Signal and Background $\cos \theta_{2}$ angular distributions. & 108
\end{tabular}

\begin{tabular}{lll}
\hline A.4 Signal and Background $\Phi$ angular distributions. & 108
\end{tabular}

\begin{tabular}{lll}
\hline A.5 Signal and Background $\Phi_{1}$ angular distributions. & 109
\end{tabular}

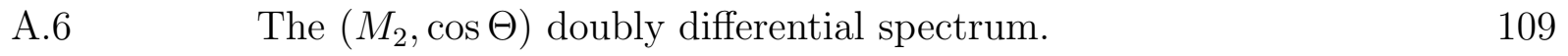

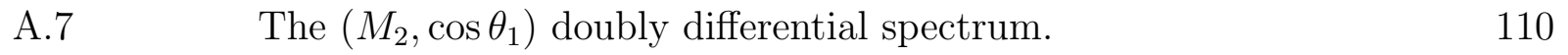

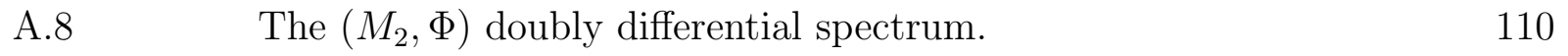

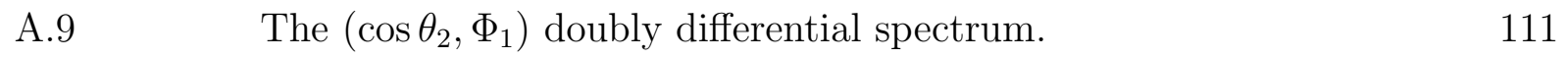

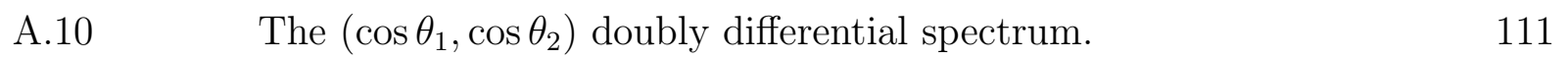

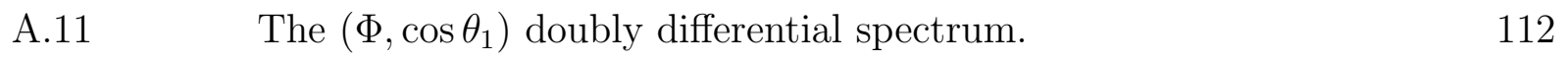

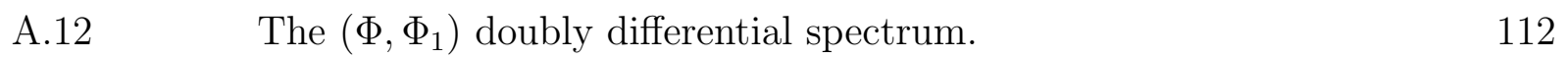




\section{CHAPTER 1}

\section{Introduction}

The recent discovery of a Higgs boson at the LHC [1, 2] with properties resembling those predicted by the Standard Model (SM) gives strong indication that the final missing

piece of the SM is now in place. In particular, the mechanism responsible for Electroweak Symmetry Breaking (EWSB) and generating masses for the $Z$ and $W$ vector bosons appears to have been established. Even with this amazing discovery there are still many outstanding theoretical and phenomenological questions which suggest that there must be physics Beyond the Standard Model (BSM). As we investigate in this thesis, the Higgs boson offers the exciting possibility of acting as a window to this new physics through various avenues which are experimentally testable in the coming years. We investigate a subset of these possibilities and begin by discussing them briefly below before a detailed examination in the following chapters.

\subsection{The Standard Model and the Need For New Physics}

In the SM [3, 4] all of the interactions among the particles are determined by $S U(3)_{c} \otimes$ $S U(2)_{W} \otimes U(1)_{Y}$ gauge invariance (along with Lorentz invariance and renormalizability). The mechanism responsible for EWSB is due to the vacuum expectation value (VEV) of a spin-0 electroweak doublet carrying hypercharge $Y=1 / 2$ which can be represented as,

$$
H \equiv(1,2,1 / 2)
$$


under $S U(3)_{c} \otimes S U(2)_{W} \otimes U(1)_{Y}$. The masses of the $W$ and $Z$ bosons follow from the scalar kinetic operator,

$$
\mathcal{L} \sim\left(D_{\mu} H\right)^{\dagger}\left(D^{\mu} H\right)
$$

after EWSB which gives the symmetry breaking pattern $S U(2)_{W} \otimes U(1)_{Y} \rightarrow U(1)_{e m}$. The masses for all fermions in the SM can be generated through Yukawa interactions of the form,

$$
\mathcal{L} \sim H \bar{f}_{L} f_{R}
$$

Thus masses for all particles in the SM can be generated with just a single electroweak doublet, with the exception of neutrinos for which no right-handed partner has been detected thus far. With the recent measurement of a Higgs mass $\sim 125 \mathrm{GeV}$ all free parameters in the SM have now been measured, making the SM fully predictive. It is a renormalizable theory so in principal there is no need for additional new physics below scales where gravitational effects become important.

However, there are both theoretical and experimental reasons to suspect there must be BSM physics. As mentioned above, in the SM there is no explanation for the experimentally observed fact that neutrinos have a non-zero mass. Since the SM does not contain a right-handed neutrino, it can not generate a mass for the neutrinos in a similar manner to the quarks and charged leptons. There is also much indirect evidence for the existence of Dark Matter (DM) which makes up the majority of the matter density in the universe. Since in the SM there is no viable DM candidate, any explanation of the nature of DM must include BSM physics. 
There are of course an endless number of ways to extend the SM to explain the many puzzles which still persist. In the final chapter of this thesis we discuss a possible extension of the SM where the accidental global symmetry in the SM associated with overall lepton number is promoted to an abelian gauge symmetry. This model provides an explanation for the generation of neutrino masses and the existence and stability of DM as well as the fact that no lepton number violating processes have been observed to date. We also examine the DM phenomenology of this model as well as its potential signatures at the LHC. In particular we study in detail how this model will affect decays of the Higgs boson and how the various partial widths might be modified from their SM predictions. There are of course many other puzzles which remain unanswered, such as solutions to the hierarchy problem, but we do not address those in this thesis.

\subsection{The Higgs as a Probe of New Physics}

The discovery of the Higgs itself offers the exciting opportunity to not only uncover the mechanism responsible for EWSB, but perhaps the first signs of BSM physics in its production and decays. As more data is collected at the LHC and experiments begin 'precision' studies on the Higgs, small deviations from the SM prediction could be the

first indications of new physics. A thorough understanding and exploration of possible new physics which could lead to deviations in the Higgs couplings to SM particles is of paramount importance. Of these couplings, those to electroweak gauge bosons are particularly important since they are a probe of the EWSB mechanism. In addition, these are an ideal place for the effects of new physics to manifests themselves, especially 
through loop effects. In this thesis we explore various aspects of the Higgs couplings to neutral electroweak gauge bosons.

In particular we examine Higgs couplings to $Z Z, Z \gamma$ and $\gamma \gamma$ gauge boson pairs which then decay to electrons $(e)$ and muons $(\mu)$; the so called 'Higgs Golden Channel'. This channel is vital to Higgs studies because it is very precisely measured at the LHC. Furthermore, because of the four body final state each event contains a wealth of information including important correlation effects which allow one to deduce detailed properties about the scalar coupling to the neutral gauge bosons. We analyze all of these correlations and in particular isolate the different interference effects which occur between the intermediate gauge bosons. Our studies are based on an analytic computation of the fully differential cross sections for both the signal and background which we present in detail.

Because of the large number of observables in each event and the precision with which it is measured the golden channel lends itself to analytic methods, which often gives greater intuition and theoretical control. This makes the golden channel ideal for studies using the Matrix Element Method (MEM) to assess the discriminating power of the golden channel at the LHC. An MEM can be used in a variety of ways such as performing a signal from background extraction [5] or, as we will see below, performing simple hypothesis testing between different signals. Once enough data is collected, one can also use an MEM to do parameter extraction of the Higgs couplings in the golden channe

This thesis is organised as follows : In Chap. 2 we present detailed analytic calculations of both signal and background fully differential cross sections in the $2 e 2 \mu$ Higgs golden channel. We also examine various $1 \mathrm{D}$ and 2D differential spectrums. In Chap. 3 we

\footnotetext{
${ }^{1}$ Currently part of ongoing work with Joe Lykken and collaborators at CMS.
} 
perform an MEM study of Higgs couplings to neutral $Z Z$ and $Z \gamma$ as a function of the number of observed events. In Chap. 4 we present an explicit model which explains a subset of the many puzzles which can not be solved within the framework of the SM. Finally in the Appendix we examine the various golden channel differential spectrums in more detail and show a variety of plots of $1 \mathrm{D}$ and $2 \mathrm{D}$ spectrums. All of the material in this thesis can be found in more detail in [6], [7], and [8]. 


\section{CHAPTER 2}

\section{Scrutinizing the Higgs Signal and Background in the $2 e 2 \mu$ Golden Channel}

\subsection{Introduction}

With the recent discovery of a new resonance at the LHC [1, 2] the focus now shifts to the determination of its detailed properties including its spin, $\mathrm{CP}$, and electroweak (EW) quantum numbers. It has been shown in recent studies [5, 6, 912 and also emphasized for quite some time [13 17], that the decay to four charged leptons is a powerful channel in accomplishing this goal. Because of the experimental precision with which this channel is measured, it offers one of the few opportunities to use analytic methods to analyze the data. We thus seek to extend previous analytic calculations of both the signal and the standard model (SM) background and present completely general, leading order (LO) fully differential cross sections for the $2 e 2 \mu$ final state mediated by intermediate $Z$ and $\gamma$ gauge bosons. In addition to performing discovery/exclusion analysis and signal hypothesis testing one could, with enough data, experimentally determine all possible couplings of a spin-0 scalar to pairs of neutral electroweak (EW) gauge bosons in one multi-parameter fit using these expressions.

Analytic expressions are ideal for use in the matrix element method (MEM) taking full advantage of all of the kinematic information in the event. One can then use the 
fully differential cross section to build a likelihood function [18, 19] to be used as a discriminant. For a recent study of the golden channel comparing existing leading order MEM-based approaches and software [20], along with providing code which calculates kinematic discriminants based on the Madgraph [21] matrix element squared see [22]. We view this 'analytic approach' as equivalent and complementary to these other approaches. These analytic expressions also allow for more flexibility in performing multidimensional fits to determine coupling values which will be useful when performing parameter extraction. Our parametrization allows for easy implementation of various hypothesis tests as well as the addition of NLO effects which can also be implemented into an MEM framework [23, 24].

For the signal we compute the fully differential decay width for the process $\varphi \rightarrow$ $Z Z+Z \gamma+\gamma \gamma \rightarrow 2 e 2 \mu$ where $\varphi$ is a spin-0 scalar. We allow for the most general $\mathrm{CP}$ odd/even mixtures and include all interference between intermediate vector bosons. While these expressions are applicable to the newly discovered boson at $125 \mathrm{GeV}$, they are also applicable for any new scalar decaying to neutral gauge bosons. This allows one to consider a variety of hypotheses which can be tested against one another. It should be emphasized however that for optimal performance, even when testing between two different signal CP and spin hypothesis, one should also include the background in the discriminant since in any given sample it is not known with full certainty which are background and which are signal events. Thus we seek to provide both signal and background distributions which can be used together to build a complete likelihood.

For the background we compute the fully differential cross section for the $q \bar{q} \rightarrow 2 e 2 \mu$ process. This includes the contributions from all the intermediate vector bosons through 
both t-channel pair production and the singly resonant four-lepton production s-channel process $q \bar{q} \rightarrow Z \rightarrow 2 e 2 \mu$. We include all interference effects between the intermediate vector bosons as well the interference between the s-channel and t-channel diagrams which can affect the differential distributions as we will see below. Also, unlike the analytic calculations in [5, 25] of the golden channel background differential cross section, these expressions are valid for a much larger energy range for the four lepton invariant mass as well as the invariant mass of each lepton pair. In particular, since these also include the $\gamma \gamma$ contribution one can probe lower values in the differential mass spectrums, which as we will see is a highly discriminating region. The intermediate vector bosons are allowed to be on or off-shell and in what follows we do not distinguish between the two. We do not discuss the $4 e$ and $4 \mu$ final states explicitly, but in some kinematic regimes the interference effects between identical final state particles can be sizable [22]. We leave an inclusion of these final states to future work.

Although other channels can also probe the tensor couplings of a resonance to neutral gauge bosons, the golden channel, with a four body final state has the advantage of extra kinematic variables, such as the azimuthal angle between lepton decay planes. This variable would be unavailable for example in the $\gamma \gamma$ final state. In addition to offering more kinematic observables, the golden channel offers the unique opportunity to test all of the possible tensor couplings including any potential interference effects between the different operators in one direct (and very precise) fully correlated measurement without any recourse to theoretical input (other than the production cross section of course). This allows for stringent tests of the SM to be performed and perhaps allow us to uncover new physics which may be hiding in subtle effects within the golden channel. 
In addition to presenting the calculation of the fully differential cross sections we examine various singly and doubly differential distributions and elucidate the subtle interference effects between the different contributions to the signal and background. Of course a proper treatment of the golden channel requires careful study of detector resolution and acceptance effects, but we leave that to ongoing analyses.

\subsection{Four Lepton Events}

The kinematics of four lepton $(4 \ell)$ events are described in detail in many places in the literature and here we use the convention found in [9]. We comment on the kinematics briefly and point out that in the case of the background the physical interpretation of the kinematic variables is not as intuitive as in the case of previous studies which only considered the t-channel $Z Z$ contribution. Now since we include the contribution from resonant four lepton production, the lepton pairs do not necessarily reconstruct to a physical particle. In this case, resonant production of a $Z$ (or possibly $\gamma$ ) is followed by decay to charged leptons one of which radiates a $Z / \gamma$, which again decays to charged leptons (see Fig. 2.4). The first lepton pair which radiates the second vector boson does not reconstruct to the $Z$ boson four momentum, which in this case is also equal to the invariant mass of the $4 \ell$ system. The kinematics remain unchanged, but now we must interpret the angles defined in the lepton pair rest frame with respect to the direction of momentum of the lepton pair system as opposed to that of one of the gauge bosons. Thus, we have the following more general interpretations for the kinematic variables defined in the $4 \ell$ rest frame;

- $M_{1,2}-$ The invariant mass of the two lepton pair systems. 
- $\Theta$ - The 'production angle' between the momentum vectors of the lepton pair which reconstructs to $M_{1}$ and the total $4 \ell$ system momentum.

- $\theta_{1,2}$ - Polar angle of the momentum vectors of $e^{-}, \mu^{-}$in the lepton pair rest frame.

- $\Phi_{1}$ - The angle between the plane formed by the $M_{1}$ lepton pair and the "production plane' formed out of the momenta of the incoming partons and the momenta of the two lepton pair systems.

- $\Phi$ - The angle between the decay planes of the final state lepton pairs in the rest frame of the $4 \ell$ system.

These variables are all independent subject to the constraint $\left(M_{1}+M_{2}\right) \leq \sqrt{s}$ where $s$ is the invariant mass squared of the $4 \ell$ system. We have also ignored the irrelevant azimuthal production angle.

In the case of the signal events one can replace 'lepton pair' momentum with $Z$ or $\gamma$ momentum since in those cases, both lepton pairs do indeed decay from a vector boson and the intuition follows that found in Fig 2.1. The same can be said for background events which proceed through t-channel pair production. In these cases, the angle $\Phi_{1}$ defines the azimuthal angle between the di-boson production plane and the plane formed by the lepton pair which reconstructs to $M_{1}$ and $\Theta$ is the vector boson production angle. Other than this more subtle interpretation of the various kinematic variable however, in practice the definitions of these variables are left unchanged from the definitions found in 9] which we follow from here on. 


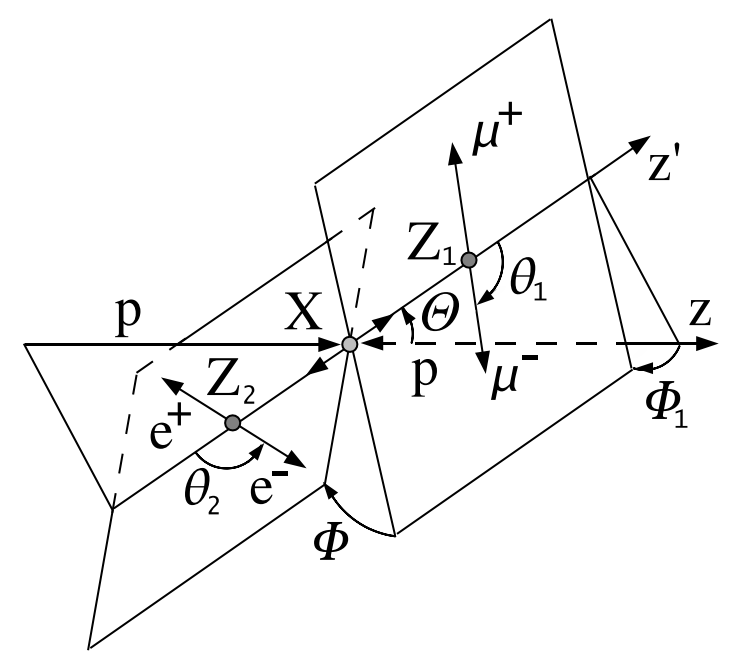

Figure 2.1. Definition of angles in the four lepton CM frame $X$.

\subsection{Signal}

In this section we present the calculation of the signal fully differential cross section and examine the differential mass spectra for several signal hypotheses. We take our signal to be a general spin-0 scalar and consider all possible couplings to any combination of $Z$ and $\gamma$ pairs allowing for mixtures of both $\mathrm{CP}$ even and odd interactions. Previous studies have analytically computed the $Z Z$ [9, 10] contribution to the golden channel, but as far as we are aware, none consider the contributions from the $Z \gamma$ and $\gamma \gamma$ intermediate states. There are also interference effects between the intermediate state which are not present when $\gamma$ is not allowed to decay. As we will see, these effects can manifest themselves in the kinematic distributions. Of course for a SM Higgs, the $Z \gamma$ and $\gamma \gamma$ contributions to the golden channel are expected to be small, but this need not be true for a general scalar or if the discovered resonance turns out to have enhanced couplings to $Z \gamma$ or to $\gamma \gamma$. How large these effects are once one takes into account detector and acceptance effects deserves careful study, but we leave this for ongoing work. 
The most general couplings of a spinless particle to two gauge bosons with four momenta $k_{1}$ and $k_{2}$ can be expressed as,

$$
\begin{aligned}
i \Gamma_{i j}^{\mu \nu}= & v^{-1}\left(A_{1 i j} m_{Z}^{2} g^{\mu \nu}+A_{2 i j}\left(k_{1} \cdot k_{2} g^{\mu \nu}-k_{1}^{\nu} k_{2}^{\mu}\right)\right. \\
& \left.+A_{3 i j} \epsilon_{\mu \nu \alpha \beta} k_{1}^{\alpha} k_{2}^{\beta}\right)
\end{aligned}
$$

where $i j=Z Z, Z \gamma$, or $\gamma \gamma$. The $A_{1,2,3}$ are dimensionless arbitrary complex form factors and $v$ is the Higgs vacuum expectation value (vev), which we have chosen as our overall normalization. For the case of a scalar coupling to $Z \gamma$ or $\gamma \gamma$ electromagnetic gauge invariance requires $A_{1}=0$, while for $Z Z$ it can be generated at tree level as in the SM or by higher dimensional operators. We have chosen to write the vertex in this form to make the connection with operators in the Lagrangian which may generate them more transparent. For example the following list of operators may generate a coupling as in Eq.(2.1),

$$
\begin{aligned}
\mathcal{L} \sim & \frac{1}{v} \varphi\left(g_{h} m_{Z}^{2} Z^{\mu} Z_{\mu}+g_{Z} Z^{\mu \nu} Z_{\mu \nu}+\tilde{g}_{Z} Z^{\mu \nu} \widetilde{Z}_{\mu \nu}\right. \\
& +g_{Z \gamma} F^{\mu \nu} Z_{\mu \nu}+\tilde{g}_{Z \gamma} F^{\mu \nu} \widetilde{Z}_{\mu \nu} \\
& \left.+g_{\gamma} F^{\mu \nu} F_{\mu \nu}+\tilde{g}_{\gamma} F^{\mu \nu} \widetilde{F}_{\mu \nu}+\ldots\right)
\end{aligned}
$$

where $Z_{\mu}$ is the $Z$ field while $V_{\mu \nu}=\partial_{\mu} V_{\nu}-\partial_{\nu} V_{\mu}$ the usual bosonic field strengths. The dual field strengths are defined as $\widetilde{V}_{\mu \nu}=\frac{1}{2} \epsilon_{\mu \nu \rho \sigma} V^{\rho \sigma}$ and the ... is for operators of dimension higher than five. For a given model many of these are of course zero. If $\varphi$ is the Standard Model Higgs, then $g_{h}=i$, while $g_{Z}, g_{Z \gamma}$ and $g_{\gamma \gamma}$ are $\neq 0$, but loop induced and small. Detailed studies of the $Z Z$ contribution to the golden channel mediated through 
the operators with coefficients $g_{h}, g_{Z}$ were conducted in [10, 11, 26]. The operators corresponding to $g_{Z \gamma}$ were studied in [6] for the golden channel final state and in [27] for the $\ell^{+} \ell^{-} \gamma$ final state and both were shown to be useful discriminators.

Other recent studies of these operators, though not only through the golden channel final state, have also been done. The pseudo scalar couplings $\tilde{g}_{Z}, \tilde{g}_{Z \gamma}, \tilde{g}_{\gamma}$ were studied recently in the context of the newly discovered resonance in [28] where it was shown that a purely CP odd scalar is disfavored as the new resonance. The analysis of [29] shows that with a fit of the $\gamma \gamma, Z Z^{*}$, and $W W^{*}$ rates, as well as the absence of a large anomaly in continuum $Z \gamma$, that the scenario of the four lepton decays being due to $g_{Z}$ or $g_{Z \gamma}$ is strongly disfavored. While these statements contain few assumptions, they are still model dependent and should be confirmed by direct measurements.

Even if the newly discovered resonance appears to be 'SM like', it is still possible that it can have contributions to the $2 e 2 \mu$ channel coming from operators other than $g_{h}$ which are slightly enhanced relative to the SM prediction. Here we are motivated by asking what information can be extracted from this channel with out any a-priori reference to other measurements or theoretical input. In addition, there still exists the possibility that another scalar resonance will be discovered which can also decay to EW gauge boson pairs. In this case it may have comparable contributions from the various operators. Thus we allow for all operators in Eq. (2.2) to contribute simultaneously including all interference effects between the $Z Z, Z \gamma$, and $\gamma \gamma$ intermediate states. Because the vertex in terms of arbitrary complex form factors is more general than the Lagrangian, for purposes of the calculation we use Eq.2.1 explicitly. Below we summarize the details of the calculation. 


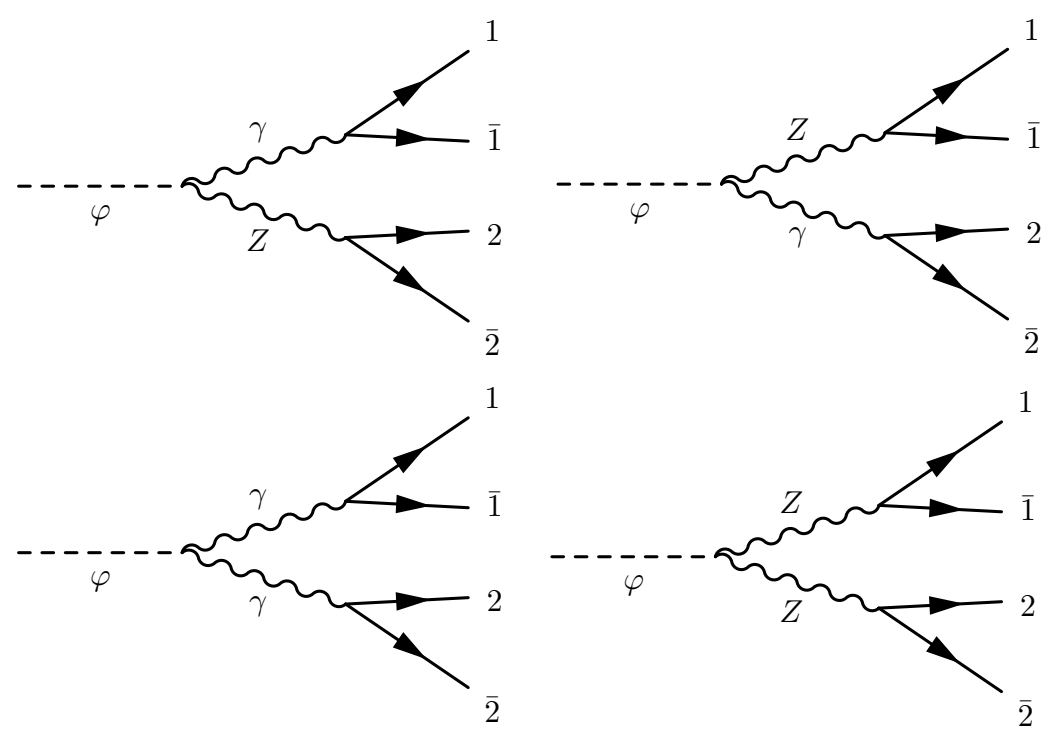

Figure 2.2. Feynman diagrams contributing to $\varphi \rightarrow 2 \ell_{1} 2 \ell_{2}$. The arrows are to indicate the direction of momentum flow.

\subsubsection{Calculation}

To compute the process $\varphi \rightarrow Z Z+Z \gamma+\gamma \gamma \rightarrow 4 \ell$ we include the diagrams shown in Fig. 2.2 and parametrize the scalar coupling to gauge bosons as in Eq. 2.1. The total amplitude can be written as,

$$
\mathcal{M}=\mathcal{M}_{Z Z}+\mathcal{M}_{Z \gamma}+\mathcal{M}_{\gamma Z}+\mathcal{M}_{\gamma \gamma}
$$

which upon squaring gives,

$$
\begin{gathered}
|\mathcal{M}|^{2}=\left|\mathcal{M}_{Z Z}\right|^{2}+\left|\mathcal{M}_{Z \gamma}\right|^{2}+\left|\mathcal{M}_{\gamma Z}\right|^{2}+\left|\mathcal{M}_{\gamma \gamma}\right|^{2} \\
+2 \operatorname{Re}\left(\mathcal{M}_{Z Z} \mathcal{M}_{Z \gamma}^{*}+\mathcal{M}_{Z Z} \mathcal{M}_{\gamma Z}^{*}+\mathcal{M}_{Z Z} \mathcal{M}_{\gamma \gamma}^{*}\right. \\
\left.\mathcal{M}_{\gamma \gamma} \mathcal{M}_{Z \gamma}^{*}+\mathcal{M}_{\gamma \gamma} \mathcal{M}_{\gamma Z}^{*}+\mathcal{M}_{Z \gamma} \mathcal{M}_{\gamma Z}^{*}\right) .
\end{gathered}
$$


An explicit calculation of all of these terms is overwhelming, but things can be simplified greatly by taking the final state leptons to be massless. In this case, the momentum dependent terms in the $Z$ boson propagator numerators do not contribute. This leads to the propagators of both $Z$ and $\gamma$ to have the same Lorentz structure, namely the Minkowski metric $g_{\mu \nu}$. This implies that all of these terms have the same general Lorentz structure. The only difference from these terms comes from Breit-wigner factors in the propagators as well as in the couplings of the vector bosons, some of which are zero thus 'turning off' the contributions from their corresponding Lorentz structure. To see this, let us consider the amplitude for any combination of intermediate $Z$ and $\gamma$ shown in Fig.2.2.

$$
\begin{gathered}
\mathcal{M}_{i j}=\bar{u}_{j}\left(i \gamma^{\gamma}\left(g_{2 R}^{j} P_{R}+g_{2 L}^{j} P_{L}\right)\right) v_{j}\left(\frac{-i g_{\nu \gamma}}{M_{2}^{2}-m_{j}^{2}+i m_{j} \Gamma_{j}}\right) \\
\Gamma_{i j}^{\mu \nu}\left(\frac{-i g_{\mu \sigma}}{M_{1}^{2}-m_{i}^{2}+i m_{i} \Gamma_{i}}\right) \bar{u}_{i}\left(i \gamma^{\sigma}\left(g_{1 R}^{i} P_{R}+g_{1 L}^{i} P_{L}\right)\right) v_{i}
\end{gathered}
$$

where $i, j$ label $Z$ or $\gamma$ while 1 and 2 label the final state leptons and can in principal be $e$ or $\mu$. In the $4 e$ and $4 \mu$ case one must also include the interference between identical particles, but we do not address that issue here. Upon squaring the amplitude and summing over final state lepton polarizations we can obtain a general amplitude squared which encompasses any of terms in Eq.2.4 and is given by,

$$
\begin{gathered}
\mathcal{M}_{i j} \mathcal{M}_{\overline{i j}}^{*}=\left(D_{1 i} D_{2 j} D_{1 \bar{i}}^{*} D_{2 \bar{j}}^{*}\right)^{-1} \\
\left(g_{\mu \sigma} \mathcal{T}_{1 i \bar{i}}^{\sigma \bar{\sigma}} g_{\overline{\mu \sigma}}\right)\left(g_{\nu \gamma} \mathcal{T}_{2 j \bar{j}}^{\gamma \bar{\gamma}} g_{\overline{\nu \gamma}}\right) \Gamma_{i j}^{\mu \nu} \Gamma_{\overline{i j}}^{* \overline{\mu \nu}}
\end{gathered}
$$


where

$$
\begin{gathered}
\mathcal{T}_{1 i \bar{i}}^{\sigma \bar{\sigma}}=\left(g_{1 R}^{i} g_{1 R}^{\bar{i}}+g_{1 L}^{i} g_{1 L}^{\bar{i}}\right) \operatorname{Tr}\left(\not p_{1} \gamma^{\sigma} \not p_{\overline{1}} \gamma^{\bar{\sigma}}\right) / 2 \\
+\left(g_{1 R}^{i} g_{1 R}^{\bar{i}}-g_{1 L}^{i} g_{1 L}^{\bar{i}}\right) \operatorname{Tr}\left(\not p_{1} \gamma^{\sigma} \not p_{\overline{1}} \gamma^{\bar{\sigma}} \gamma^{5}\right) / 2 \\
D_{1 i}=M_{1}^{2}-m_{i}^{2}+i \Gamma_{i} m_{i}
\end{gathered}
$$

and $\Gamma_{i j}^{\mu \nu}$ are given in Eq. 2.1. . The $g_{R, L}^{i}$ are at this point general left and right handed couplings of a ' $Z$-like' spin-1 vector boson to a pair of fermions. The bars are to indicate that the corresponding index belongs to the conjugated amplitude and are distinct indices from the un-bared ones. We treat all couplings at every vertex encountered when tracing over the Dirac strings as distinct as well as all Breit-Wigner factors so for any amplitude squared term there can in principal be four different vector bosons as intermediate states. In the case of the photon we have of course $g_{R}^{\gamma}=g_{L}^{\gamma}=-e_{e m}$ and $m_{\gamma}=\Gamma_{\gamma}=0$. Since at this stage the various couplings and masses are completely general, Eq.2.6 applies to any process where a scalar decays to two spin-1 vector bosons which then decay to massless fermions through 'Z-like' couplings.

Expanding out the terms in Eq.22.6 we can write the amplitude squared as,

$$
\begin{gathered}
\mathcal{M}_{i j} \mathcal{M}_{i \overline{i j}}^{*}=\mathcal{C}_{i j \overline{i j}}^{++} L_{i j \overline{i j}}^{++}+\mathcal{C}_{i j \overline{i j}}^{+-} L_{i j \overline{i j}}^{+-}+ \\
\mathcal{C}_{i j \overline{i j}}^{-+} L_{i j \overline{i j}}^{-+}+\mathcal{C}_{i j \overline{i j}}^{--} L_{i j \overline{i j}}^{--}=\sum_{a b} \mathcal{C}_{i j \overline{i j}}^{a b} L_{i j \overline{i j}}^{a b}
\end{gathered}
$$

where $a, b=(+,-)$ with $a$ and $b$ corresponding to the fermion pairs labeled 1 and 2 respectively and

$$
\begin{gathered}
\mathcal{C}_{i j \overline{i j}}^{ \pm \pm}=\frac{\left(g_{1 R}^{i} g_{1 R}^{\bar{i}} \pm g_{1 L}^{i} g_{1 L}^{\bar{i}}\right)\left(g_{2 R}^{j} g_{2 R}^{\bar{j}} \pm g_{2 L}^{j} g_{2 L}^{\bar{j}}\right)}{4\left(D_{1 i} D_{2 j} D_{1 \bar{i}}^{*} D_{2 \bar{j}}^{*}\right)} \\
L_{i j \overline{i j}}^{ \pm \pm}=\left(g_{\mu \sigma} T_{1 \pm \pm}^{\sigma \bar{\sigma}} g_{\overline{\mu \sigma}}\right)\left(g_{\nu \gamma} T_{2 \pm}^{\gamma \bar{\gamma}} g_{\overline{\nu \gamma}}\right) \Gamma_{i j}^{\mu \nu} \Gamma_{\overline{i j}}^{* \overline{\mu \nu}} .
\end{gathered}
$$


The $T_{1 \pm}^{\sigma \bar{\sigma}}$ are the Dirac traces found in Eq. 2.7) and \pm indicates whether the trace ends with a $\gamma^{5}(-)$ or not $(+)$. The full amplitude squared can then be built out of the object: $1^{1}$ in Eq. 2.9.,

$$
\left(\mathcal{M}_{i j} \mathcal{M}_{\overline{i j}}^{*}\right)^{a b}=\mathcal{C}_{i j \overline{i j}}^{a b} L_{i j \overline{i j}}^{a b}
$$

Since all of the angular information is contained in the $L_{i j \overline{i j}}^{a b}$ we can take advantage of the simple nature of these terms to perform the desired integration before summing over \pm and the various vector boson intermediate states, after which analytic integration becomes unmanageable. Expressions for the $L_{i j \bar{j} j}^{a b}$ are obtained in terms of invariant dot products and CM variables. These objects can be used to build the differential cross section of any scalar decay to four massless fermions via two spin-1 vector bosons. From these one can also reproduce analytic results for other processes such as the semi-leptonic decay of the Higgs to $\ell \nu j j[30$.

The final fully differential decay width can now be written as,

$$
\frac{d \Gamma_{\varphi}}{d M_{1}^{2} d M_{2}^{2} d \Omega}=\Pi_{4 \ell} \sum_{a b}\left(\sum_{i \overline{i j}} \mathcal{C}_{i j \overline{i j}}^{a b} L_{i j \overline{i j}}^{a b}\right)
$$

where $d \Omega=d c_{\Theta} d c_{\theta_{2}} d c_{\theta_{1}} d \Phi d \Phi_{1}\left(c_{\theta}=\cos \theta\right)$ and $\Pi_{4 \ell}$ is the final state lepton four body phase space derived following [31] and given by,

$$
\begin{aligned}
& \Pi_{4 \ell}=\left(\frac{1}{2 \pi}\right)^{2}\left(\frac{1}{32 \pi^{2}}\right)^{2}\left(\frac{1}{32 \pi s}\right) \\
& \cdot\left(1+\frac{\left(M_{1}^{2}-M_{2}^{2}\right)^{2}}{s^{2}}-\frac{2\left(M_{1}^{2}+M_{2}^{2}\right)}{s}\right)^{1 / 2} .
\end{aligned}
$$

We can obtain the differential mass spectrum $2^{2}$ via,

$$
\frac{d \Gamma_{\varphi}}{d M_{1}^{2} d M_{2}^{2}}=\Pi_{4 \ell} \sum_{a b}\left(\sum_{i j \overline{i j}} \mathcal{C}_{i j \overline{i j}}^{a b}\left(\int d \Omega L_{i j \overline{i j}}^{a b}\right)\right)
$$

\footnotetext{
$\overline{{ }^{1} \text { Expressions }}$ for the various coefficients and Lorentz structure can be obtained by emailing the corresponding author.

${ }^{2}$ We give an analytic expression for a particular hypothesis in the Appendix.
} 
We note that we perform the sum over vector bosons before the sum over \pm which allows for greater simplification of the expressions. We can now go on to examine the differential mass spectrum for different signal hypothesis. In the Appendix we show various singly and doubly differential spectra for a number of signal hypotheses. We also give in Eq.A.1 of the Appendix, an explicit expression for the doubly differential mass spectrum of a scalar with SM-like $Z Z$ couplings and both $\mathrm{CP}$ even and $\mathrm{CP}$ odd $Z \gamma$ couplings including all interference effects.

\subsubsection{The Differential Mass Spectra}

In this section we examine the singly differential mass spectra for various signal hypotheses and give a feel for how $M_{1}$ and $M_{2}$ might be able to distinguish between the different operators in Eq. 2.2. Explicitly we consider the following cases ${ }^{3}$

- 1: SM including $Z \gamma$ and $\gamma \gamma\left(A_{1 Z Z}=2, A_{2 Z \gamma}=0.007, A_{2 \gamma \gamma}=-0.008\right)^{4}$

- 2: SM coupling to $Z Z$ plus enhanced $Z \gamma$ and $\gamma \gamma\left(A_{1 Z Z}=2, A_{2 Z \gamma}=6 * 0.007, A_{2 \gamma \gamma}=\right.$ $-1.3 * 0.008)$

- 3: $\mathrm{SM}$ coupling to $Z Z$ plus $\mathrm{CP}$ odd couplings to $\gamma \gamma$ and $Z \gamma\left(A_{1 Z Z}=2, A_{3 Z \gamma}=\right.$ $\left.0.01, A_{3 \gamma \gamma}=0.01\right)$

- 4: $\mathrm{CP}$ odd/even mixed coupling to $Z Z$ only $\left(A_{1 Z Z}=2, A_{3 Z Z}=0.1\right)$

- 5: General Scalar $\left(A_{1 Z Z}=0.1, A_{2 Z Z}=1, A_{2 Z \gamma}=0.01, A_{2 \gamma \gamma}=0.01, A_{3 Z Z}=1, A_{3 Z \gamma}=\right.$ $\left.0.01, A_{3 \gamma \gamma}=0.01\right)$

where we also show the values for the couplings chosen in Eq. 2.1. Couplings whose values are not shown in a given hypotheses are taken to be zero and we take all values at $\sqrt{s}=m_{h}=125$ $\mathrm{GeV}$. Note that all of these couplings can be interpreted in terms of the couplings in Eq.2.2. if we assume only up to dimension 5 operators contribute.

\footnotetext{
${ }^{3}$ We have validated these cases with FeynRules/CalcHEP [20, 32] and the Monte Carlo generator introduced in [9].

${ }^{4}$ Values obtained from [29] after translating to our parametraziation.
} 
We obtain the differential mass spectra via Eq.(2.13), followed by integration over $M_{1}$ or $M_{2}$, and compare them for different hypotheses. These are shown in Fig. 2.3 for two ranges. The first range we take $4 \mathrm{GeV}<M_{1,2}<120 \mathrm{GeV}$ treating $M_{1}$ and $M_{2}$ symmetrically shown in the top plot. In this case we only show the $M_{1}$ distribution since it is identical to the $M_{2}$ distribution and only show the lower mass region above which the different cases are very similar.

We also consider the more 'experimental' cut requiring a wide window around the $Z$ boson mass $40 \mathrm{GeV}<M_{1}<120 \mathrm{GeV}$ and $4 \mathrm{GeV}<M_{2}<120 \mathrm{GeV}$ for the 'off-shell' vector boson. In this case the $M_{1}$ distribution is indistinguishable for the separate cases so we only show the $M_{2}$ distribution. One can see, that in particular in the low mass region, these variables can be highly discriminating between the different cases. We point out also that, if values of $M_{2} \lesssim 10 \mathrm{GeV}$ can be probed, the requirement of a window around a $Z$ boson may decrease sensitivity to certain hypotheses which have a sizable $\gamma \gamma$ or $Z \gamma$ component such as hypothesis 5.

Our lower bound on $M_{2}$ is chosen to be $4 \mathrm{GeV}$ since lower values of $M_{2}$ runs the risk of contamination from $J / \psi$ states whose mass is $\sim 3 \mathrm{GeV}$. We emphasize that experimental analyses should be made to push down as far possible since as can be seen in Fig. 2.3, one needs to be able to probe $M_{2}$ below $\sim 10 \mathrm{GeV}$ in order to discriminate between a SM scalar (hypothesis 1) and one with enhanced $Z \gamma$ and $\gamma \gamma$ couplings (hypothesis 2) for example. Though current experimental signal searches in the golden do not yet consider such low values for $M_{2}$, it seems feasible to push the $M_{2}$ cut down further as was done in the CMS observation of the $Z \rightarrow \ell^{+} \ell^{-} \ell^{+} \ell^{-}$process [33]. We therefore include this highly interesting region here and hope that it may motivate efforts to push the $M_{2}$ reach lower. We leave a complete analysis including detector effects to an ongoing study. 

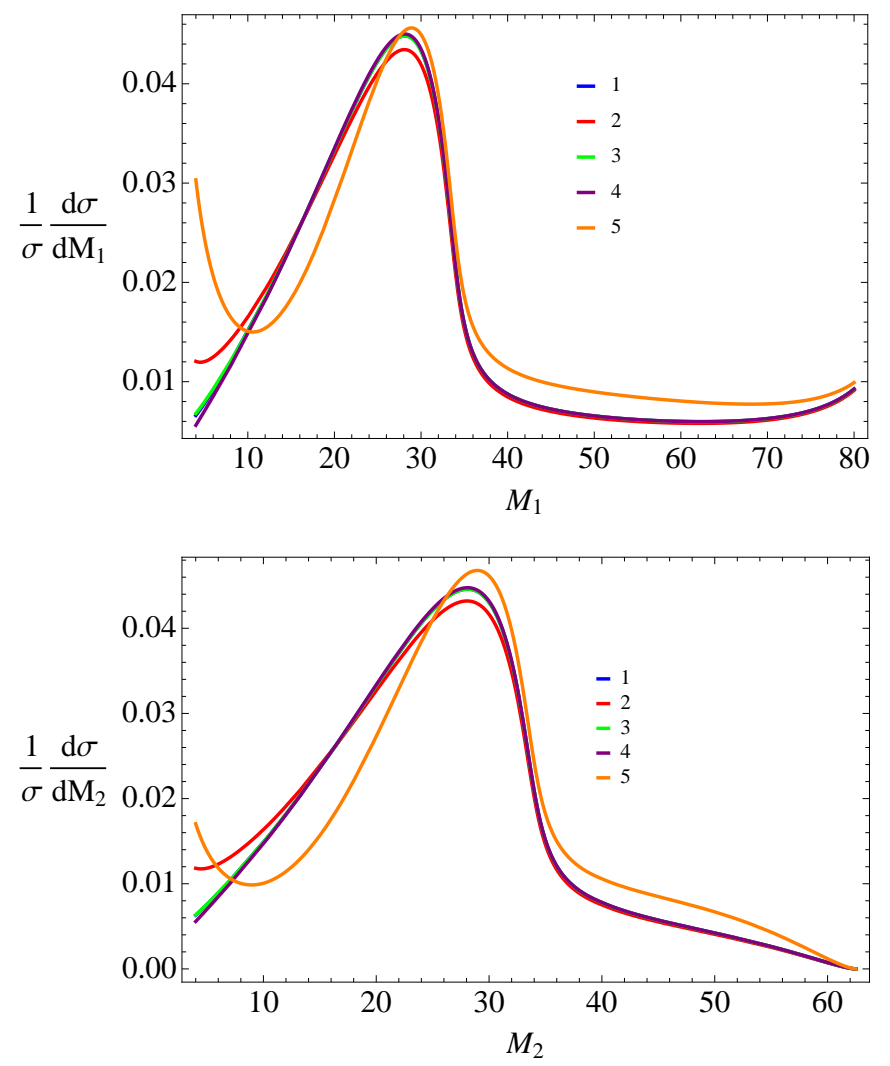

Figure 2.3. In the top plot we take $4 \mathrm{GeV}<M_{1,2}<120 \mathrm{GeV}$ while in the bottom plot we take the range $40 \mathrm{GeV}<M_{1}<120 \mathrm{GeV}, 4 \mathrm{GeV}$ $<M_{2}<120 \mathrm{GeV}$ (assuming $M_{1}>M_{2}$ ) at $\sqrt{s}=m_{h}=125 \mathrm{GeV}$ when integrating over phase space. The SM is shown in blue, but is essentially indistinguishable from hypothesis 3 (see text).

\subsection{Background}

The dominant irreducible background to the golden channel comes from $q \bar{q}$ annihilation into gauge bosons. At energies $\sim 125 \mathrm{GeV}$ the dominant contribution comes from t-channel $Z \gamma$ production. However, as we will see contributions from s-channel $Z \rightarrow 4 \ell$ diagrams can effect the angular distributions such as the distribution of the angle between the decay planes $\Phi$ defined in Sec 3.1. Furthermore, we include the $Z Z$ and $\gamma \gamma$ contributions since in principal these are always present and may have observable interference effects due to the fact that they 
add at the amplitude level when decaying to charged leptons. In addition, the inclusion of these contributions allows for considering a much larger energy range in one fully differential cross section than can be considered when including only the $t$ and $u$ channel contributions. Of course NLO effects, including the $g g$ initiated process [34 36] will contribute as well, but these are expected to be small and mainly only effect the 'input' invariant mass (and overall normalization) for the fully differential cross sections. We will examine this point below.

It should also be noted that ideally one would like to include the $4 e$ and $4 \mu$ final states which in some kinematic regimes can have non-negligible contributions from interference between final state particles [22]. The inclusion of this channel would allow for greater sensitivity for the same amount of luminosity. However, because of the interference between identical final states in this case, the Lorentz structure becomes severely more complicated and we thus leave this calculation for future work.

\subsubsection{Calculation}

The background calculation is much more involved than the signal calculation due to a higher number of Feynman diagrams in addition to a more complicated Lorentz structure. As in the signal case the amplitude can be written as,

$$
\mathcal{M}=\mathcal{M}_{Z Z}+\mathcal{M}_{Z \gamma}+\mathcal{M}_{\gamma Z}+\mathcal{M}_{\gamma \gamma}
$$

Now however, each of these amplitudes breaks down into six 'sub-amplitudes'. To see this, let us first consider the $Z Z$ mediated decays. There are three diagrams which contribute to the $2 e 2 \mu$ process shown in Fig. 2.4. First there is the t-channel contribution shown in the bottom diagram. This contribution (and its u-channel counterpart) has been computed previously for both on-shell [25] and off-shell [5] $Z$ bosons. The second contribution comes from resonant $2 e 2 \mu$ production proceeding through the top two diagrams. Each of these diagrams also has 


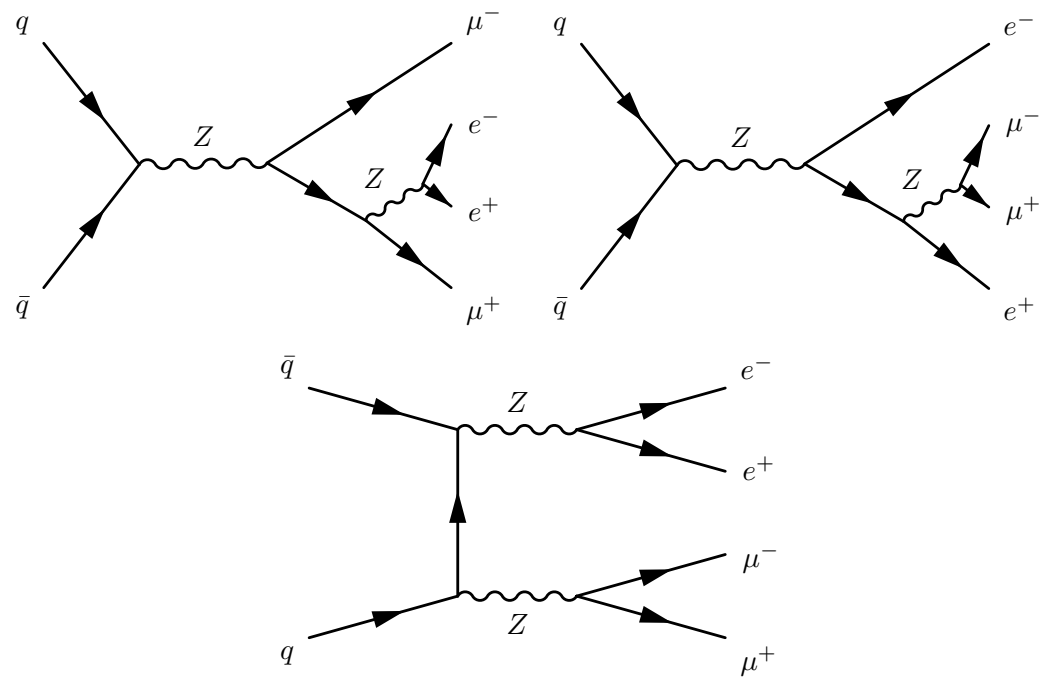

Figure 2.4. Feynman diagrams contributing to $q \bar{q} \rightarrow Z Z \rightarrow 2 e 2 \mu$ and $q \bar{q} \rightarrow$ $Z \rightarrow 2 e 2 \mu$. The arrows are to indicate the direction of momentum flow.

a corresponding 'crossed' diagram taking into account the other possibility for attaching the vector boson lines. This gives six diagrams for the $Z Z$ contribution to the golden channel. Similarly, there are six more for the $\gamma \gamma$ contribution plus six for $Z \gamma$ and six for $\gamma Z$ giving a total of twenty four diagrams. At first this many diagrams can seem intractable, but as we will see, when organized in a proper manner the calculation is relatively straightforward with the help of Tracer [37] to perform the Lorentz contraction.

To begin we first note that the six diagrams can be 'twisted' and arranged into the form found in Fig. 2.5 where we now allow the vector bosons to take on any $Z$ or $\gamma$, but once chosen are treated as fixed. We use the conventions indicated in the diagrams and in particular refer to the diagrams $(a),(c)$, and $(e)$ as 't-channel' type diagrams and $(b),(d)$, and $(f)$ as 'u-channel'. This is not to be confused with the typical vocabulary for this process which refers to diagrams $(a)$ and $(b)$ as $\mathrm{t}$ and $\mathrm{u}$ channel and diagrams $(c)-(f)$ as s-channel. We find this re-naming convenient for organizing and reducing the many terms which need to be computed for the differential cross section. The Lorentz structure for all of these amplitudes is clearly the same. 


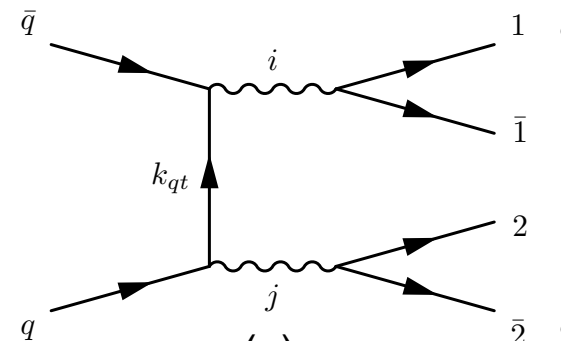

(a)

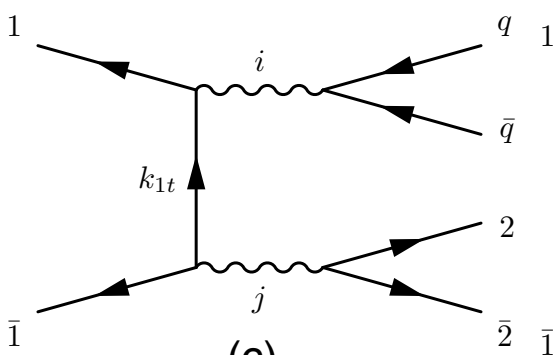

(c)

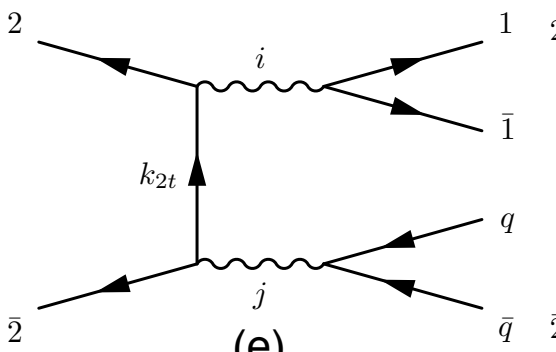

(e)

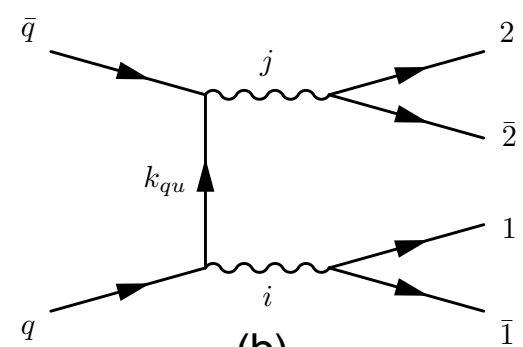

(b)

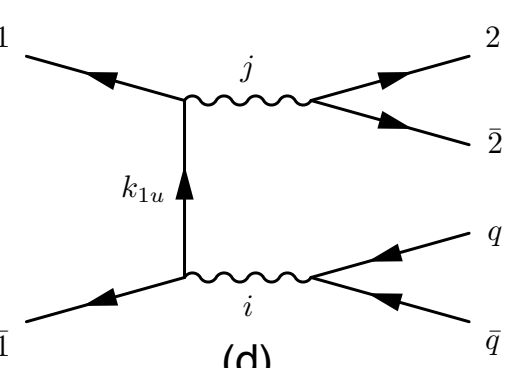

(d)

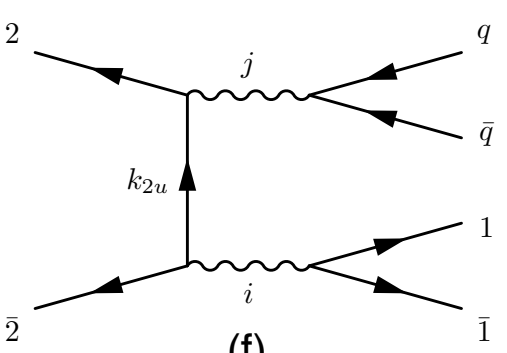

(f)

Figure 2.5. Feynman diagrams contributing to $q \bar{q} \rightarrow V_{i} V_{j} \rightarrow 2 \ell_{1} 2 \ell_{2}$ and $q \bar{q} \rightarrow V_{i} \rightarrow 2 \ell_{1} 2 \ell_{2}$. Note that diagrams $(c)-(f)$ are in fact s-channel diagrams so the fermions labeled by 1 and 2 are not to be confused as being in the initial state. This is taken into account in how the various momenta are assigned as indicated by the arrows.

One needs only to keep proper track of how the various momentum are routed through each diagram. We can see this by considering the amplitude explicitly. Using the massless initial quark and final state lepton approximation we can write any of the amplitudes in Fig. 2.5 as,

$$
\begin{gathered}
\mathcal{M}_{X i j}^{n}=\bar{u}_{Z}\left(i \gamma^{\sigma}\left(g_{Z R}^{j} P_{R}+g_{Z L}^{j} P_{L}\right)\right) v_{Z}\left(\frac{-i g_{\mu \sigma}}{M_{Z}^{2}-m_{j}^{2}+i m_{j} \Gamma_{j}}\right) \\
\bar{v}_{X}\left(i \gamma^{\mu}\left(g_{X R}^{i} P_{R}+g_{X L}^{i} P_{L}\right)\right)\left(\frac{i \not k X_{X n}}{k_{X n}^{2}}\right)\left(i \gamma^{\nu}\left(g_{X R}^{j} P_{R}+g_{X L}^{j} P_{L}\right)\right) u_{X} \\
\left(\frac{-i g_{\nu \gamma}}{M_{Y}^{2}-m_{i}^{2}+i m_{i} \Gamma_{i}}\right) \bar{u}_{Y}\left(i \gamma^{\gamma}\left(g_{Y R}^{i} P_{R}+g_{Y L}^{i} P_{L}\right)\right) v_{Y}
\end{gathered}
$$


where we label the amplitude by the 'long' dirac string, in this case $X$. The labels $X / Y / Z=$ $1,2, q$ where 1,2 are for final state lepton pairs while $q$ is for the initial state quarks. The $i, j=Z, \gamma$ label the vector bosons and $n=t, u$ labels the $\mathrm{t}$ and $\mathrm{u}$-channel diagrams in our new vocabulary. The invariant masses are defined as $M_{Y}^{2}=\left(p_{Y}+p_{\bar{Y}}\right)^{2}$. The internal fermion momentum are given in terms of external momentum by,

$$
\begin{aligned}
& k_{q t}=p_{q}-\left(p_{1}+p_{\overline{1}}\right), k_{q u}=p_{q}-\left(p_{2}+p_{\overline{2}}\right) \\
& k_{1 t}=-p_{\overline{1}}-\left(p_{2}+p_{\overline{2}}\right), k_{1 u}=\left(p_{q}+p_{\bar{q}}\right)-p_{\overline{1}} \\
& k_{2 t}=\left(p_{q}+p_{\bar{q}}\right)-p_{\overline{2}}, k_{2 u}=-p_{\overline{2}}-\left(p_{1}+p_{\overline{1}}\right) .
\end{aligned}
$$

Note that the invariant masses $M_{Y}$ and $M_{Z}$ do not necessarily correspond to the invariant mass formed by the final state lepton pairs, as they do in the signal case and in previous analytic calculations of the golden channel background which neglect the s-channel diagrams. Now with the inclusion of the resonant four lepton processes in $(c)-(f)$ we have for these diagrams $M_{Y / Z}^{2}=M_{q}^{2}=s$ which is equal to the invariant mass of the four lepton system. To obtain any of the physical amplitudes one simply assigns the appropriate labels to Eq.(2.15) as well as the appropriate momenta. Thus for example for diagram (c) we have $X \rightarrow 1, Y \rightarrow q, Z \rightarrow 2$, and $n \rightarrow t$. To switch from t-channel type to $\mathrm{u}$-channel diagrams (staying in the same row in Fig 2.5 one simply takes $t \rightarrow u$ and $\gamma^{\sigma} \leftrightarrow \gamma^{\gamma}$. Of course at this stage all these labels are arbitrary meaning that the amplitude in Eq. (2.15) applies to any process with this topology and Lorentz structure. Note that for the $Z$ propagators we drop the momentum dependent terms since they do not contribute in the massless lepton approximation. As mentioned in the signal case, for the photon $g_{R}^{\gamma}=g_{L}^{\gamma}$ and $m_{\gamma}=\Gamma_{\gamma}=0$, but for now we take the couplings and propagators as general. 
As in the case of the signal, the next step is to find a generalized amplitude squared for any two of the six diagrams. Although there are in principal thirty six terms when squaring the amplitudes, these organize themselves into only two distinct types of Lorentz structure. The first type is found when multiplying any two diagrams in the same row of Fig. 2.5. This is the Lorentz structure found in our previous calculations of the $Z Z$ contribution in which only diagrams $\mathcal{M}_{q t}$ and $\mathcal{M}_{q u}$ are included (the top row). The square of $\mathcal{M}_{1 t}+\mathcal{M}_{1 u}$ and $\mathcal{M}_{2 t}+\mathcal{M}_{2 u}$ (second and third rows) will also exhibit this Lorentz structure. The second type of Lorentz structure is obtained when taking the product of any two diagrams in different rows. In the conventional language, interference between the first and second row or first and third row corresponds to interference between the t-channel di-boson production amplitudes and the s-channel diagrams. Interference between the second and third row corresponds to interference between the two types of s-channel diagrams. We first discuss the 'squared' terms where the amplitudes are contained within the same row before examining the interference terms between rows.

Using the conventions just described, we can write the product of any two amplitudes within a row as

$$
\begin{gathered}
\mathcal{M}_{X i j}^{n} \mathcal{M}_{X \overline{i j}}^{m *}=\left(D_{Y i} D_{Z j} D_{Y \bar{i}}^{*} D_{Z \bar{j}}^{*}\right)^{-1} \\
\left(g_{\mu \sigma} \mathcal{T}_{Y i \bar{i}}^{\sigma \bar{\sigma}} g_{\overline{\mu \sigma}}\right)\left(g_{\nu \gamma} \mathcal{T}_{Z j \bar{j}}^{\gamma \bar{\gamma}} g_{\overline{\nu \gamma}}\right) \mathcal{T}_{X i j \bar{\mu} \bar{j} n m}^{\nu \mu \bar{\nu}}
\end{gathered}
$$

where the $\mathcal{T}_{Y i \bar{i}}^{\sigma \bar{\sigma}}$ and $D_{Y i}$ are defined similarly to those in Eq.2.7) and the long Dirac string is given by,

$$
\begin{gathered}
\mathcal{T}_{X i \bar{j} \bar{j} n m}^{\nu \mu \bar{\mu} \nu}=\left(g_{X R}^{i} g_{X R}^{j} g_{X R}^{\bar{i}} g_{X R}^{\bar{j}}+g_{X L}^{i} g_{X L}^{j} g_{X L}^{\bar{i}} g_{X L}^{\bar{j}}\right) / 2 \\
\cdot \operatorname{Tr}\left(\not p_{X} \gamma^{\nu} \not k_{X n} \gamma^{\mu} \not p_{X} \gamma^{\bar{\mu}} \not k_{X m} \gamma^{\bar{\nu}}\right)+ \\
\left(g_{X R}^{i} g_{X R}^{j} g_{X R}^{\bar{i}} g_{X R}^{\bar{j}}-g_{X L}^{i} g_{X L}^{j} g_{X L}^{\bar{i}} g_{X L}^{\bar{j}}\right) \\
\cdot \operatorname{Tr}\left(\not p_{X} \gamma^{\nu} \not k_{X n} \gamma^{\mu} \not p_{\bar{X}} \gamma^{\bar{\mu}} \not k_{X m} \gamma^{\bar{\nu}} \gamma^{5}\right) / 2 .
\end{gathered}
$$


Expanding out the terms in Eq.2.17) we can organize in a manner similar to Eq.2.8) and write the amplitude squared as,

$$
\mathcal{M}_{X i j}^{n} \mathcal{M}_{X i j}^{m *}=\sum_{a b c} \mathcal{C}_{X X i j \overline{i j}}^{a b c} L_{X X n m}^{a b c}
$$

where again $a, b, c=(+,-)$ in the order $X, Y, Z$ and

$$
\begin{gathered}
\mathcal{C}_{X X i j \overline{i j}}^{ \pm \pm \pm}=\left(8 D_{Y i} D_{Z j} D_{Y \bar{i}}^{*} D_{Z \bar{j}}^{*}\right)^{-1} \\
\left(g_{X R}^{i} g_{X R}^{j} g_{X R}^{\bar{i}} g_{X R}^{\bar{j}} \pm g_{X L}^{i} g_{X L}^{j} g_{X L}^{\bar{i}} g_{X L}^{\bar{j}}\right) \\
\cdot\left(g_{Y R}^{i} g_{Y R}^{\bar{i}} \pm g_{Y L}^{i} g_{Y L}^{\bar{i}}\right)\left(g_{Z R}^{j} g_{Z R}^{\bar{j}} \pm g_{Z L}^{j} g_{Z L}^{\bar{j}}\right) \\
L_{X X n m}^{ \pm \pm \pm}=\left(g_{\mu \sigma} T_{Y \pm}^{\sigma \bar{\sigma}} g_{\overline{\mu \sigma}}\right)\left(g_{\nu \gamma} T_{Z \pm}^{\gamma \bar{\gamma}} g_{\overline{\nu \gamma}}\right) T_{X n m \pm}^{\nu \mu \bar{\mu} \nu} \cdot
\end{gathered}
$$

The $T_{Y, Z \pm}^{\sigma \bar{\sigma}}$ are the Dirac traces found in Eq. 2.7 while the $T_{X n m \pm}^{\nu \mu \bar{\mu} \nu}$ are those found in 2.18 . Again \pm indicates whether the trace ends with a $\gamma^{5}(-)$ or not $(+)$. We note that unlike in the signal case, when organized in this way (essentially by powers of $\gamma^{5}$ ) the gauge structure completely factors from the Lorentz structure. This allows us to sum over all possible intermediate vector bosons at this stage to write,

$$
\begin{gathered}
\mathcal{M}_{X}^{n} \mathcal{M}_{X}^{m *}=\sum_{i j \overline{i j}} \mathcal{M}_{X i j}^{n} \mathcal{M}_{X \overline{i j}}^{m *} \\
=\sum_{i j \overline{i j}} \sum_{a b c} \mathcal{C}_{X X i j \overline{i j}}^{a b c} L_{X X n m}^{a b c} \\
=\sum_{a b c}\left(\sum_{i j \overline{i j}} \mathcal{C}_{X X i j \overline{i j}}^{a b c}\right) L_{X X n m}^{a b c}=\sum_{a b c} \mathcal{C}_{X X}^{a b c} L_{X X n m}^{a b c} .
\end{gathered}
$$

This simplifies things greatly and in particular the objects $\mathcal{C}_{X X}^{a b c}$ now contain all of the information regarding the intermediate vector bosons including the interference effects between the different processes. These will serve as overall coefficients for the various Lorentz structure pieces. 
We are now in a position to examine the interference terms. Let us take the product of any two diagrams not in the same row. One can show explicitly,

$$
\begin{gathered}
\mathcal{M}_{X i j}^{n} \mathcal{M}_{Y \overline{i j}}^{m *}=\left(D_{Y i} D_{Z j} D_{Z \bar{i}}^{*} D_{X \bar{j}}^{*}\right)^{-1} \\
\left(g_{\mu \sigma} \mathcal{T}_{X i \bar{\gamma} \bar{j} n}^{\nu \mu \bar{\nu}} g_{\overline{\nu \gamma}}\right)\left(g_{\nu \gamma} \mathcal{T}_{Y i \overline{i j} m}^{\overline{\mu \nu} \sigma} g_{\overline{\mu \sigma}}\right) \mathcal{T}_{Z j \bar{i}}^{\gamma \bar{\sigma}}
\end{gathered}
$$

where the $\mathcal{T}_{Z i \bar{i}}^{\gamma \bar{\sigma}}$ are as before and the new Dirac strings are given by,

$$
\begin{gathered}
\mathcal{T}_{X i j \bar{j} n}^{\nu \mu \bar{\gamma}}=\left(g_{X R}^{i} g_{X R}^{j} g_{X R}^{\bar{j}}+g_{X L}^{i} g_{X L}^{j} g_{X L}^{\bar{j}}\right) / 2 \\
\cdot \operatorname{Tr}\left(\not p_{X} \gamma^{\nu} \not k_{X n} \gamma^{\mu} \not p_{\bar{X}} \gamma^{\bar{\gamma}}\right)+ \\
\left(g_{X R}^{i} g_{X R}^{j} g_{X R}^{\bar{j}}-g_{X L}^{i} g_{X L}^{j} g_{X L}^{\bar{j}}\right) \\
\cdot \operatorname{Tr}\left(\not p_{X} \gamma^{\nu} \not k_{X n} \gamma^{\mu} \not p_{\bar{X}} \gamma^{\bar{\gamma}} \gamma^{5}\right) / 2 .
\end{gathered}
$$

The distinct Lorentz structure found here as compared to that found in Eq.2.17) is due to the different path taken when tracing over the fermonic strings.

Again we expand out the terms in Eq.2.22 to obtain,

$$
\mathcal{M}_{X i j}^{n} \mathcal{M}_{Y \overline{i j}}^{m *}=\sum_{a b c} \mathcal{C}_{X Y i j \overline{i j}}^{a b c} L_{X Y n m}^{a b c}
$$

where,

$$
\begin{gathered}
\mathcal{C}_{X Y i j \overline{i j}}^{ \pm \pm \pm}=\left(8 D_{Y i} D_{Z j} D_{Z \bar{i}}^{*} D_{X \bar{j}}^{*}\right)^{-1}\left(g_{Z R}^{j} g_{Z R}^{\bar{i}} \pm g_{Z L}^{j} g_{Z L}^{\bar{i}}\right) \\
\cdot\left(g_{X R}^{i} g_{X R}^{j} g_{X R}^{\bar{j}} \pm g_{X L}^{i} g_{X L}^{j} g_{X L}^{\bar{j}}\right)\left(g_{Y R}^{i} g_{Y R}^{\bar{i}} g_{Y R}^{\bar{j}} \pm g_{Y L}^{i} g_{Y L}^{\bar{i}} g_{Y L}^{\bar{j}}\right) \\
L_{X Y n m}^{ \pm \pm \pm}=\left(g_{\mu \sigma} T_{X n}^{\nu \mu \bar{\gamma}} g_{\overline{\nu \gamma}}\right)\left(g_{\nu \gamma} T_{Y m}^{\overline{\mu \nu} \sigma} g_{\overline{\mu \sigma}}\right) T_{Z}^{\gamma \bar{\sigma}} .
\end{gathered}
$$

and the $T_{X n}^{\nu \mu \bar{\gamma}}$ are the traces found in Eq. 2.23). As mentioned above, since the gauge and Lorentz structure factor completely we are free to perform the sum over the intermediate vector bosons 
at this stage once again to obtain the various Lorentz structure coefficients,

$$
\begin{gathered}
\mathcal{M}_{X}^{n} \mathcal{M}_{Y}^{m *}=\sum_{i j \overline{i j}} \mathcal{M}_{X i j}^{n} \mathcal{M}_{Y \overline{i j}}^{m *}= \\
\sum_{a b c}\left(\sum_{i j \overline{i j}} \mathcal{C}_{X Y i j \overline{i j}}^{a b c}\right) L_{X Y n m}^{a b c}=\sum_{a b c} \mathcal{C}_{X Y}^{a b c} L_{X Y n m}^{a b c} .
\end{gathered}
$$

Thus again all of the information concerning the intermediate vector bosons is contained in $\mathcal{C}_{X Y}^{a b c}$. We now have all of the pieces $5^{5}$ necessary to build the total amplitude squared of the diagrams in Fig. 2.5 including all contributions from the intermediate vector bosons. Explicitly we have,

$$
\begin{gathered}
\left|\mathcal{M}_{q}+\mathcal{M}_{1}+\mathcal{M}_{2}\right|^{2} \\
=\sum_{a b c n m} \sum_{a b c}\left(\left(\mathcal{C}_{q q}^{a b c} L_{q q n m}^{a b c}+\mathcal{C}_{11}^{a b c} L_{11 n m}^{a b c}+\mathcal{C}_{22}^{a b c} L_{22 n m}^{a b c}\right)\right. \\
\left.+2 \operatorname{Re}\left(\mathcal{C}_{q 1}^{a b c} L_{q 1 n m}^{a b c}+\mathcal{C}_{12}^{a b c} L_{12 n m}^{a b c}+\mathcal{C}_{2 q}^{a b c} L_{2 q n m}^{a b c}\right)\right)
\end{gathered}
$$

where the sum over intermediate vector bosons has been already implicitly performed and the sum over $n, m$ which includes the $\mathrm{t}$ and $\mathrm{u}$ channel contributions is shown explicitly (note that this also factors from the vector boson sum). The $\mathcal{C}_{X Y}^{a b c}$ coefficients are in general complex due to the factor of $i$ multiplying the decay width in the massive vector boson propogators. The Lorentz structure is either purely real or purely imaginary depending on whether the term contains an even or odd number of traces ending in $\gamma^{5}$. These traces give an overall factor of $i$ (and an epsilon tensor). Thus if $L_{X Y n m}^{a b c}$ contains an even number of these traces, then it is purely real and if it contains an odd number it is purely imaginary. The squared Lorentz structure $L_{X X n m}^{a b c}$ however is strictly real as are the squared coefficients $\mathcal{C}_{X X}^{a b c}$. Taking this into account, we can

\footnotetext{
$\overline{{ }^{5} \text { Expressions }}$ for the various coefficients and Lorentz structure can be obtained by emailing the corresponding author.
} 
write for Eq.2.27 the final amplitude squared as,

$$
\begin{aligned}
& \left|\mathcal{M}_{4 \ell}\right|^{2}=\left|\mathcal{M}_{q}+\mathcal{M}_{1}+\mathcal{M}_{2}\right|^{2} \\
& =\frac{1}{2} \sum_{a b c}^{e v e n}\left(\mathcal{C}_{q q R}^{a b c} L_{q q R}^{a b c}+\mathcal{C}_{11 R}^{a b c} L_{11 R}^{a b c}+\mathcal{C}_{22 R}^{a b c} L_{22 R}^{a b c}\right) \\
& +\sum_{a b c}^{e v e n}\left(\mathcal{C}_{q 1 R}^{a b c} L_{q 1 R}^{a b c}+\mathcal{C}_{12 R}^{a b c} L_{12 R}^{a b c}+\mathcal{C}_{2 q R}^{a b c} L_{2 q R}^{a b c}\right) \\
& -\sum_{a b c}^{o d d}\left(\mathcal{C}_{q 1 I}^{a b c} L_{q 1 I}^{a b c}+\mathcal{C}_{12 I}^{a b c} L_{12 I}^{a b c}+\mathcal{C}_{2 q I}^{a b c} L_{2 q I}^{a b c}\right)
\end{aligned}
$$

where we have now performed the sum over $t$ and $u$ channel diagrams and $\mathcal{C}_{X Y R, I}^{a b c}=\mathcal{C}_{X Y}^{a b c} \pm \mathcal{C}_{X Y}^{* a b c}$ respectively. We have also implicitly included a factor of $1 / 4$ from averaging over initial state quark spins and a color factor of $1 / 3$. The sums labeled even $\equiv(+++,+--,-+-,--+)$ indicate terms with even powers of $\gamma^{5}$ and those with odd $\equiv(-++,+-+,++-,---)$ indicate terms with odd powers of $\gamma^{5}$. Note that since the photon has vector like couplings where $g_{L}=g_{R}$ all coefficients $\mathcal{C}_{X Y}^{a b c}$ with $a, b$, or $c \equiv-$ are zero for the $\gamma \gamma$ intermediate state. Thus $\gamma \gamma$ only contributes to the $\mathcal{C}_{X Y}^{+++}$coefficients (including of course when $X \equiv Y$ ).

Previous calculations of the golden channel background, which include only the di-boson production process, are contained within the first term $\mathcal{C}_{q q}^{a b c} L_{q q}^{a b c}$ of Eq. 2.28). All the other terms arise from the resonant four lepton production process and the interference between it and the di-boson production process. Note that Eq. 2.28) is also more general than for just the golden channel. In principal this expression holds for any process with the same topology and ' $Z$-like' couplings to fermions. Since we have built the expression out of a generalized Lorentz structure with coefficients, it can easily be adapted to consider new physics contributions which may enter with the same topology and alter some of the coefficients by an observable amount. Thus one can imagine performing stringent tests of the SM using this parametrization to extract the various Lorentz structure coefficients. We leave an investigation of this to future work. 
The final fully differential cross section ${ }^{6}$ is again obtained by combining the amplitude squared with the invariant four body phase space (see Eq. 2.12),

$$
\frac{d \sigma_{4 \ell}}{d M_{1}^{2} d M_{2}^{2} d \Omega}=\Pi_{4 \ell}\left|\mathcal{M}_{4 \ell}\right|^{2}
$$

The differential mass spectrum 7 is obtained again via,

$$
\frac{d \sigma_{4 \ell}}{d M_{1}^{2} d M_{2}^{2}}=\Pi_{4 \ell} \int d \Omega\left|\mathcal{M}_{4 \ell}\right|^{2}
$$

We now examine how the various components of the background contribute to the differential mass spectrum.

\subsubsection{The Differential Spectra}

In this section we examine how the individual components of the background contribute to the invariant mass spectrum of the four lepton system. In addition we also study how including parton distribution functions ( $p d f s$ ) and NLO corrections change the differential spectra by comparing normalized projections obtained from our analytic expression to Monte Carlo generated by POWHEG [38 41] and Madgraph [21].

We first separate the background into its various components which we define as the following,

- A: s-channel $2 e 2 \mu$ process

- B: $t+u$-channel $\gamma \gamma$

- C: $t+u$-channel $Z Z$

- D: $t+u$-channel $Z \gamma$

\footnotetext{
${ }^{6}$ This expression has been validated with the Madgraph matrix element squared.

${ }^{7}$ An analytic expression for the dominant component to the background is given in Eq.A.3 of the Appendix.
} 
- $\mathbf{E}: t+u$-channel $Z Z / Z \gamma / \gamma \gamma$ interference only

- $\mathbf{F}: Z Z+Z \gamma+\gamma \gamma s / t$-channel interference only

where now $s, t$, and $u$ are used in the usual sense and the resonant s-channel $2 e 2 \mu$ process can proceed through any combination of $Z$ and $\gamma$. We first consider the relative fractions of these components as a function of the invariant mass of the four lepton system for the range $100-600$ GeV in Fig. 2.6. The dotted lines indicate when a contribution is negative, which of course only occurs for interference terms in certain energy ranges when the interference is destructive. The solid black line at constant value of 1 is the total partonic level $q \bar{q} \rightarrow 2 e 2 \mu(q=u, d)$ background including all interference and all intermediate vector bosons. From Fig. 2.6 one can see how the relative contributions coming from the different components change as a function of energy.

Component $C$ (the $Z Z t+u$ channel) is the only piece of the background to have been previously calculated analytically [5, 25]. This makes up the dominant contribution above the $Z Z$ threshold, but is negligible from $110 \mathrm{GeV}<\sqrt{s}<140 \mathrm{GeV}$ and in fact is even smaller than the interference terms. We also plot the spectrum if one requires a window around the $Z$ boson mass in the bottom plot of Fig. 2.6. The dominant component near the resonance mass of $125 \mathrm{GeV}$ is $D$ regardless of the window on the $Z$ mass. Except for component $F$, one can see that the relative fractions are fairly insensitive to the $Z$ window requirement except in the range $\sim 100-110 \mathrm{GeV}$.

The flexibility of the analytic expressions also allow us to easily isolate the contribution coming from interference terms. Component $E$ for example is due to the interference between the intermediate gauge bosons in the $t+u$ channel and is destructive over the entire range regardless of the $Z$ window. The interference between the resonant s-channel and the t-channel pair production processes is shown in $F$ and switches between constructive and destructive if one requires a window around the $Z$, but otherwise is constructive. Though these components are small it is possible for them to have subtle effects on the angular distributions such as in the 

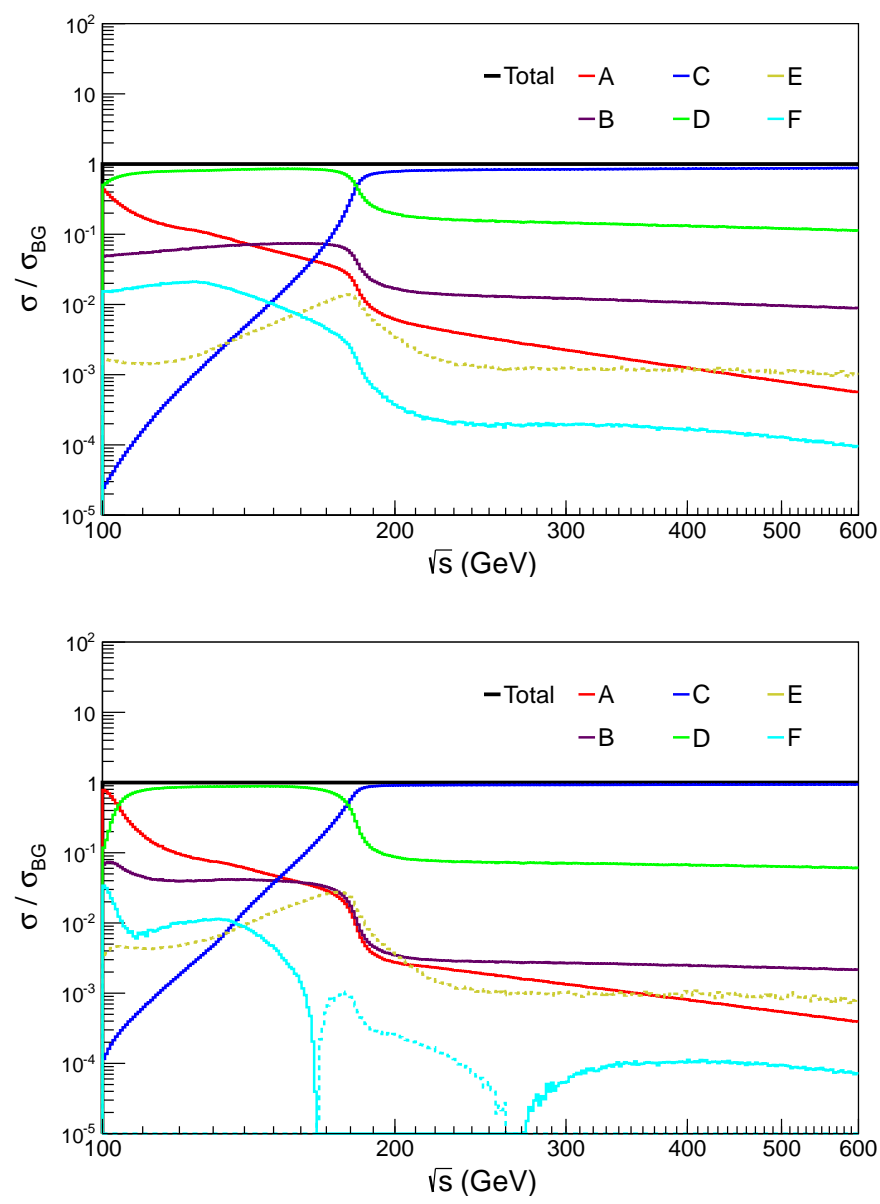

Figure 2.6. The four lepton system invariant mass spectrum (without $p d f s$ ) for the various components defined in the text. The dotted lines indicate when the interference between components is destructive, thus giving a negative contribution. In the top plot we take the ranges $4 \mathrm{GeV}<M_{1,2}<$ $120 \mathrm{GeV}$ while in the bottom plot we take the range $40 \mathrm{GeV}<M_{1}<$ $120 \mathrm{GeV}$ and $10 \mathrm{GeV}<M_{2}<120 \mathrm{GeV}$ while taking $M_{1}>M_{2}$.

modulation of the azimuthal angle $\Phi$ (See Fig. A.4) and may be particularly interesting to study in the range $100 \mathrm{GeV} \lesssim \sqrt{s} \lesssim 110 \mathrm{GeV}$. The expressions for most of the components themselves are too cumbersome to write here, but in the Appendix we give the expression for the doubly differential $\left(M_{1}, M_{2}\right)$ mass spectrum of the full $t+u$ (the sum of $B-E$ ) component which as we can see in Fig. 2.6 and Fig. 2.7 provides a very good approximation above $\sqrt{s} \sim 110 \mathrm{GeV}$. 
To examine the effects of NLO contributions and $p d f s$ we compare our parton level result for $q \bar{q} \rightarrow 2 e 2 \mu(q=u, d)$ to Monte Carlo data generated by the NLO POWEG and LO Madgraph codes which include $p d f s$ [42]. For this we define our phase space as $40 \mathrm{GeV}<M_{1}<120 \mathrm{GeV}$ and $10 \mathrm{GeV}<M_{2}<120 \mathrm{GeV}$ for the energy range $110 \mathrm{GeV}<\sqrt{s}<140 \mathrm{GeV}$. We also plot the $t+u$ component only (defined as the sum of $B-E$ ) to examine what affects neglecting the resonant $2 e 2 \mu$ process has.

In Fig. 2.7, 2.8, and 2.9 we show the kinematic distributions where it can be seen that NLO and $p d f$ contributions affect the normalized spectra negligibly. In addition we can see that neglecting the resonant process also has little effect on all the kinematic variables except $\Phi$, where it affects the modulation and in the forward regions of $\cos \theta_{1}$. As we will see in the Appendix, the modulation is due almost entirely to the resonant process. These distributions simply reflect the fact that the various kinematic distributions are not highly correlated with $\sqrt{s}$ allowing us to take $\sqrt{\hat{s}}$ essentially as an input from the $p d f s$. To build a complete hadronic differential cross section one could convolve the $\sqrt{s}$ spectrum obtained from Madgraph or POWHEG with the partonic differential cross section obtained analytically. This of course is what would be done for an LHC analysis, but we do not do that here and instead simply integrate our partonic differential cross section over $\sqrt{\hat{s}}$.

From Figs. 2.6 and 2.7 we expect the doubly differential spectrum obtained from the $t+u$ component only to be a good approximation which could be useful for a simplified analysis. We give an explicit expression for this component in Eq. A.3 of the Appendix. Though it does not use all of the kinematic variables, it should still have strong discriminating power and can be used with the methods proposed in [12] to form a powerful simplified study. 

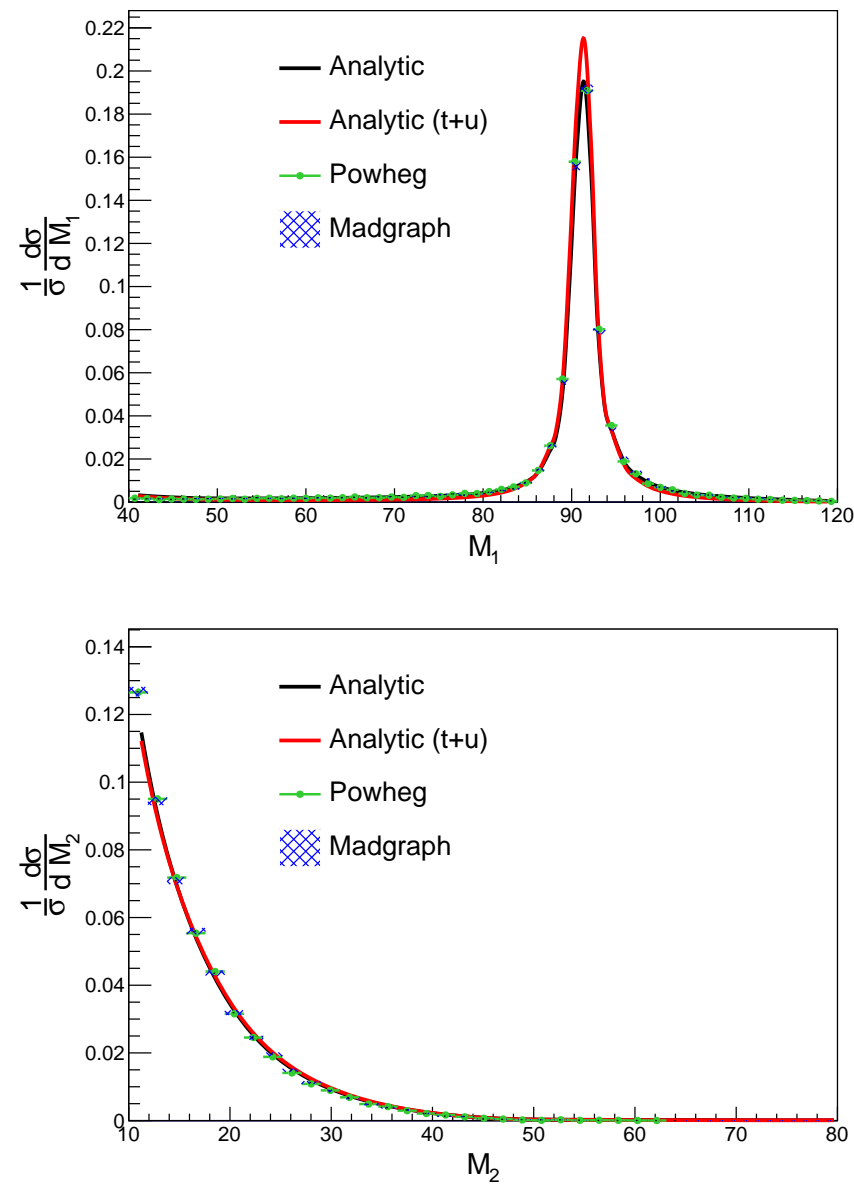

Figure 2.7. Comparing the LO and NLO results for the $M_{1}$ and $M_{2}$ invariant mass spectra for the ranges $40 \mathrm{GeV}<M_{1}<120 \mathrm{GeV}$ and $10 \mathrm{GeV}<M_{2}<$ $120 \mathrm{GeV}$. We take the range of the four lepton system invariant mass to be $110<\sqrt{s}<140 \mathrm{GeV}$.

\subsection{Conclusions and Outlook}

We have calculated and presented analytic fully general differential cross sections for the golden channel signal and background in the $2 e 2 \mu$ final state including all intermediate vector bosons and interference effects. We have presented various singly and doubly differential spectra and examined how the different interference effects manifest themselves in these distributions and in correlations between the different kinematic variables. We have also emphasized the 

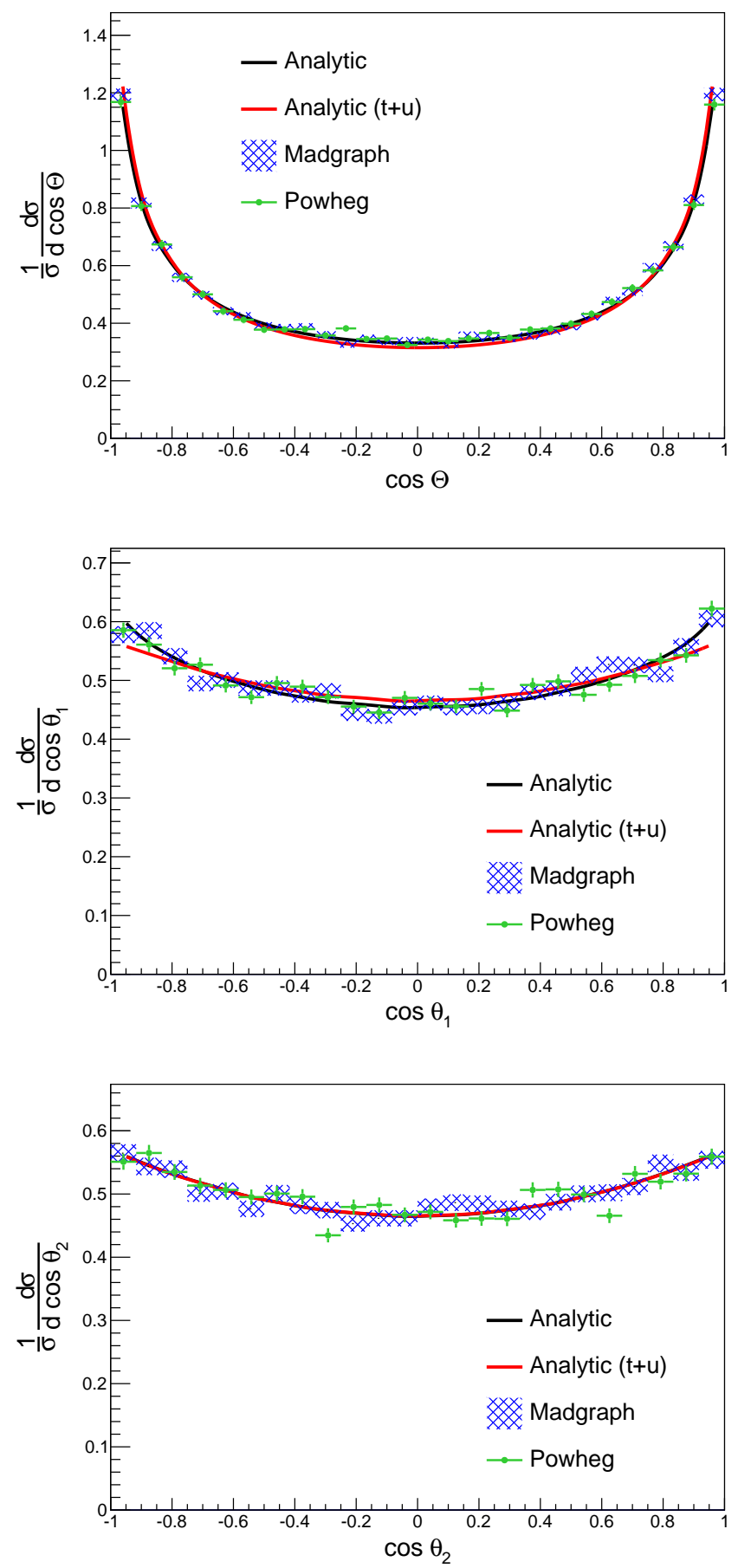

Figure 2.8. Comparing the LO and NLO results for the polar angles $\cos \Theta, \cos \theta_{1}, \cos \theta_{2}$ for the ranges $40 \mathrm{GeV}<M_{1}<120 \mathrm{GeV}$ and $10 \mathrm{GeV}$ $<M_{2}<120 \mathrm{GeV}$. We take the range of the four lepton system invariant mass to be $110<\sqrt{s}<140 \mathrm{GeV}$. 

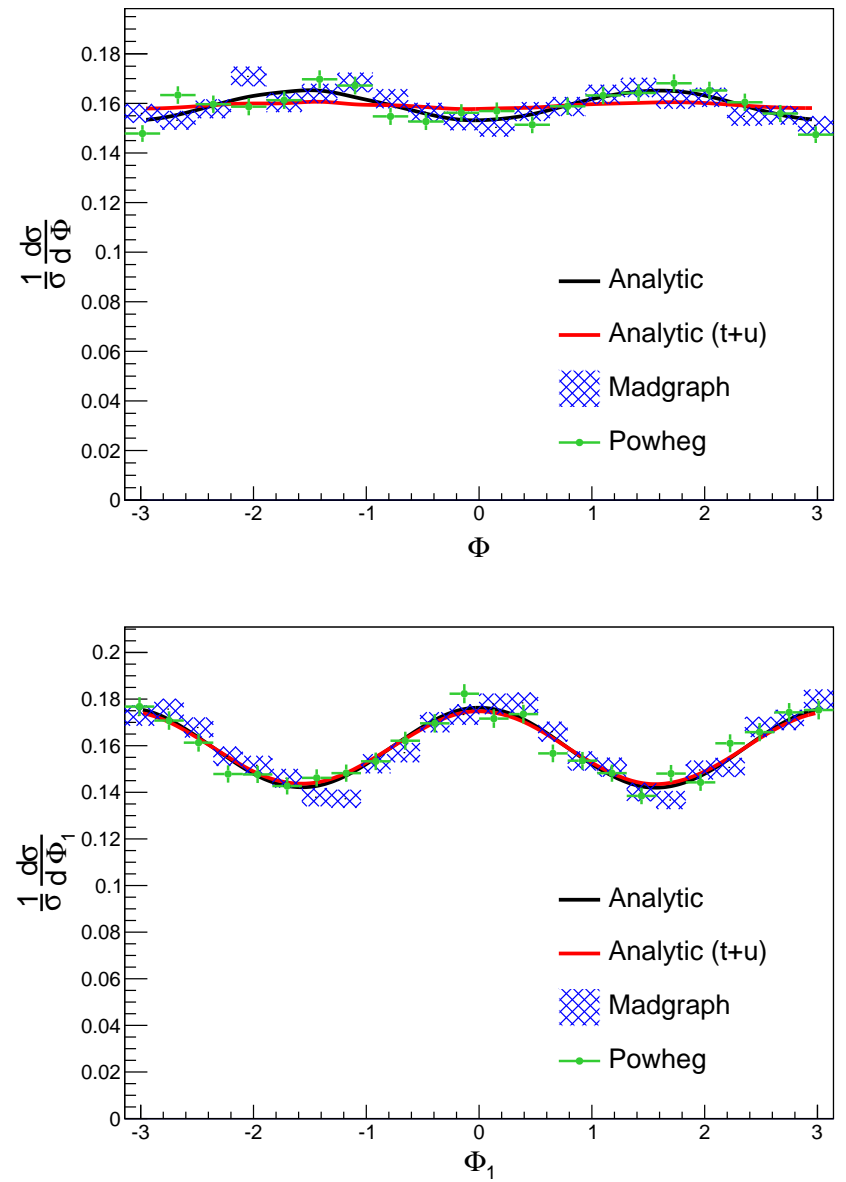

Figure 2.9. Comparing the LO and NLO results for the azimuthal angles $\Phi$ and $\Phi_{1}$ for the ranges $40 \mathrm{GeV}<M_{1}<120 \mathrm{GeV}$ and $10 \mathrm{GeV}<M_{2}<$ $120 \mathrm{GeV}$. We take the range of the four lepton system invariant mass to be $110<\sqrt{s}<140 \mathrm{GeV}$.

need to push the 'off-shell' invariant mass $\left(M_{2}\right)$ reach as low as possible as well as relaxing the ' $Z$-window' to maximize the discriminating power when testing different signal hypothesis. We have shown that the expressions can aid in distinguishing between different signal hypotheses and because signal and background are provided, both can be included into one likelihood, as should be done when performing simple hypothesis tests of different signals. These expressions 
can be implemented into an MEM analysis to perform detailed studies of the spin and CP properties of any scalar resonance which has been or may be discovered at the LHC. 


\section{CHAPTER 3}

\section{Directly Measuring the Tensor Structure of the Scalar Coupling to Gauge Bosons}

In this chapter we demonstrate the usefulness of the golden channel by performing a simple hypothesis test between a subset of the scalar tensor operators considered in Sec. 2, We do not attempt an analysis of signal extraction from a background as we performed in a study to examine heavy Higgs bosons [5], but instead assume an already purified signal sample. We attempt to delve into the signal events themselves and see what information can be learned about the new particle via a direct measurement in the golden channel. We take the hypothesis that the new particle is a parity even scalar and try to see if this channel can be used to directly measure the tensor structure of the coupling of this particle to the four lepton final state. If we denote the new scalar by $\phi$, it can have the following couplings to $Z Z$

$$
\frac{1}{v}\left(a_{h} m_{Z}^{2} \phi Z_{\mu} Z^{\mu}+a_{s} \phi Z^{\mu \nu} Z_{\mu \nu}+\ldots\right)
$$

where $Z_{\mu}$ is the $Z$ field while $Z_{\mu \nu}=\partial_{\mu} Z_{\nu}-\partial_{\nu} Z_{\mu}$. Here $v=246 \mathrm{GeV}$ is the Standard Model (SM) Higgs vev which is chosen to normalize the operators, and the ... is for operators of dimension higher than five. If $\phi$ is the Standard Model Higgs, then $a_{h}=i$, and the other coupling is loop induced and small.

As we are trying to determine whether this new particle is the SM Higgs, we must consider other possibilities. If $\phi$ does not give mass to the $Z$, then its linear coupling to gauge bosons can

proceed via the field strength tensor, $Z_{\mu \nu}$ as in the operator $a_{s}$ in Eq. (3.1). The $a_{s}$ operator 
is generically loop induced and its coefficient is model dependent. We see this sort of operator even in the Standard Model Higgs' coupling to $\gamma \gamma$ and $Z \gamma$ :

$$
\frac{1}{v}\left(a_{\gamma} \phi F^{\mu \nu} F_{\mu \nu}+a_{Z \gamma} \phi Z^{\mu \nu} F_{\mu \nu}+\ldots\right)
$$

where we continue to use $\phi$ to denote our scalar, and $F_{\mu \nu}$ is the field strength tensor for the photon. In the SM, $a_{\gamma}$ and $a_{Z \gamma}$ are induced by loops with top and $W$ giving the largest contributions. If $\phi$ is not the Higgs, then a plausible alternative is that it decays to four leptons via $a_{s}$ or $a_{Z \gamma}$. Generically, $a_{s}, a_{Z \gamma}$ and $a_{\gamma}$ are all present and of comparable size, and all three operators can mediate four lepton final states. Typically experimental searches require that the invariant mass of the one of the lepton pairs is near the $Z$ pole, so the contribution of $a_{\gamma}$ is small, but we will see that both $a_{s}$ and $a_{Z \gamma}$ need to be considered.

Bounds on all the operators in Eqs. (3.1) and (3.2) can be set using the absence of single production of this resonance at LEP [43]. In order to interpret these bounds at the LHC, however, the production cross section of this scalar must be computed, and that is a priori unknown. If $\phi$ does couple dominantly via $a_{h}$ that would be evidence that it is indeed a Higgs. On the other hand, it could still be something more exotic such as a dilation [44 46]. The crucial point is that if we are going determine if $\phi$ gives mass to the $Z$ boson, we must show that its coupling to $Z Z^{*}$ is dominantly through $a_{h}$. As we will show below, the kinematic distributions of the four lepton events can discriminate $a_{h}$ from $a_{s}$ and $a_{Z \gamma}$.

The question of distinguishing these operators via kinematics was considered briefly in [26]. We here extend their analysis by studying all possible kinematic variables which can distinguish different possible decay operators. Furthermore, the analysis of [26] only considers a mass of a scalar greater than twice the $Z$ mass, while we here will be working in the kinematic regime where one of the $Z$ 's is far off-shell. In [10], they use kinematic methods to distinguish two different 
kinds of parity even scalars, but both of their possibilities are still responsible for giving mass to the $W$ and $Z$. In other words, they both have significant $a_{h}$ in the language of Eq. (3.1).

If the new resonance has anomalous couplings to $Z$ and $W$, then the production of the resonance through vector boson fusion (VBF) would also be modified. These effects were studied in [43], where it was shown that angular correlations between the two tagging jets in VBF can constrain the value of the operators in Eq. (3.1). Here, we only consider decays of the resonance, but these two types of measurements can be complementary in fully characterizing the nature of the new state.

\subsection{Differential Spectrums}

We compute tree level analytic expressions for the full differential decay width in terms of frame invariant 4 -vector dot products before choosing the frame with kinematic variables as described in 2, We plot normalized one dimensional distributions for $\Phi, \cos \theta_{i}$, and $M_{2}$ in Fig. 3.1. We take $m_{\phi}=125 \mathrm{GeV}$ here and throughout. Distributions will change little with variations of $m_{\phi}$ within the experimental resolution. We restrict $M_{i}$ to the ranges described below in order to better approximate the experimental searches. We consider the operators $a_{h}$, $a_{s}$ and $a_{Z \gamma}$ turning on one operator at a time with the others set to zero.

In general, all three operators, $a_{h}, a_{s}$, and $a_{Z \gamma}$ will be non-zero. In the case of a Higgs-like state which gives mass to the $Z, a_{s}$ and $a_{Z \gamma}$ are loop suppressed and $a_{h}$ will dominate. If the new state does not contribute to electroweak symmetry breaking, then $a_{h}$ will often be negligible. If $a_{s} \sim a_{Z \gamma}$, which is typically the case if the two operators are generated by loops of electroweak charged matter, then the effects of $a_{Z \gamma}$ will dominate. This is because in the allowed region for $M_{2}$, a photon will be much closer to on-shell than a $Z$, and because the $Z$ has suppressed couplings to leptons relative to the photon. Even if $a_{s}$ is ten times larger than $a_{Z \gamma}, a_{Z \gamma}$ will dominate the decay and we can consider turning on just $a_{Z \gamma}$ as a reasonable approximation. On 

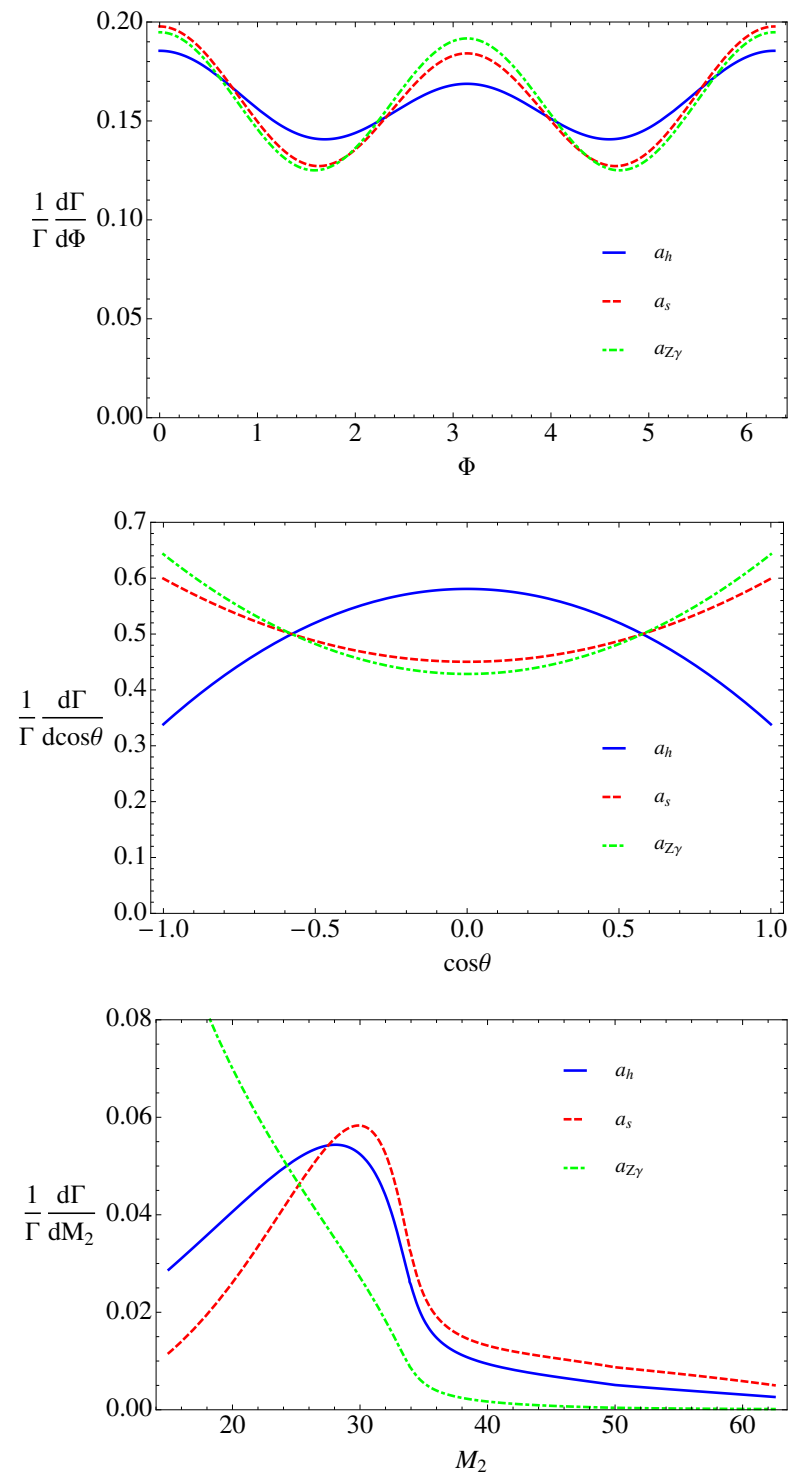

Figure 3.1. Normalized distributions for $\Phi$ (top), $\cos \theta_{i}$ (middle), and $M_{2}$ (bottom) for $m_{\phi}=125 \mathrm{GeV}$. Each plot shows curves from our three different scenarios with $a_{h}$ blue (solid), $a_{s}$ red (dashed), and $a_{Z \gamma}$ green (dot-dashed).

the other hand, one could imagine a model where $a_{Z \gamma}$ is very small, possibly due to tuning, and we therefore consider turning on only $a_{s}$ as a stand in for this possibility. From this analysis, we see that in most of the parameter space, one operator will dominate over the other two, which is why we consider scenarios where only one operator is turned on at a time. 
The top two panels in Fig. 3.1 show that the angular distributions, particularly that of $\cos \theta$ provide good discriminating power between a Higgs-like scenario $a_{h}$, and the two non-Higgs-like possibilities. The third plot shows that the $M_{2}$ distribution is different for all three scenarios, and the difference is even more pronounced for small values of $M_{2}$. This can be seen from the following simple analysis. For $a_{h}$, the matrix element goes to a constant as $M_{2} \rightarrow 0$, and a phase space factor of $M_{2} d M_{2}$ makes the rate go to zero. For $a_{s}$, the matrix element goes as $M_{2}$ for small $M_{2}$ because of the derivative in the operator, so $d \Gamma$ falls as $M_{2}^{3}$. Finally, for $a_{Z \gamma}$, the matrix element goes as $1 / M_{2}$ because the photon propagator in the denominator and the derivative in the numerator, and thus the rate goes as $1 / M_{2}$. As we will see below, realistic detector cuts such as those on lepton $p_{T}$ will change this low $M_{2}$ behavior, but this simple analysis shows that if the experiments could push down the $M_{2}$ reach of the events, they would gain discriminatory power.

We do not include a plot for $M_{1}$ because in all scenarios, it looks similar with a large peak at $M_{Z}$ that has width of $\Gamma_{Z}$. The $M_{1}$ distribution does, however, provide some discrimination power in that the number of events well below $M_{Z}$ differs for our three different scenarios. For example, in the $a_{h}$ scenario, $70 \%$ of the events will lie more than $2 \Gamma_{Z}$ away from $M_{Z}$, while the corresponding fraction for $a_{s}\left(a_{Z \gamma}\right)$ is $64 \%$ (84\%). The majority of these non-resonant events have $M_{1}<M_{Z}$.

If the four lepton events are dominated by $a_{Z \gamma}$, then there should also be decays to on-shell photons. It has been pointed out that searching for the Higgs in decays to $Z \gamma$ is a promising channel [27]. While there is as yet no direct limit in this channel, [29] uses the measurement of the $Z \gamma$ cross section to place a limit on the ratio of the $Z \gamma$ mode to the four lepton mode to be about 40. Given this, we take the $Z \gamma$ mode to be an unlikely possibility, but we still believe in checking the data to see if it can be directly excluded. 
In order to compare to experiment, we also generate Monte Carlo (MC) events. We use the Johns Hopkins MC described in [9] to simulate $a_{h}$ and $a_{s}$, and Madgraph 5 [21] for $a_{Z \gamma}$. We generate $g g \rightarrow \phi \rightarrow 4 \ell$ events where $\ell=e, \mu$ at the LHC with $\sqrt{s}=8 \mathrm{TeV}$. Gluon fusion is the dominant mode of Higgs production at the LHC [47]. Since our variables are mostly sensitive to decay and not production, the errors introduced by ignoring sub-dominant production modes will be small. We require our events to contain four charged leptons $(e$ or $\mu)$ with

- $p_{T}>10 \mathrm{GeV}$

- $|\eta|<2.5$

- $50 \mathrm{GeV}<M_{1}<110 \mathrm{GeV}$

- $M_{2}>15 \mathrm{GeV}$,

which roughly mimics the experimental selection criteria in [1, 2]. Histograms for the distinguishing kinematic variables from generated events are overlaid on the analytic results in Figs. 3.2 and 3.3 . Because the experimental resolution for energy and direction of leptons is so precise, we do not apply any smearing to the events. While a truly realistic study will need to take into account experimental reality, we here see how far the experiments could get with just the geometric cuts above.

In Fig. 3.2, we plot the $\cos \theta_{1}$ and $\cos \theta_{2}$ distributions for 1000 generated Monte Carlo events which pass the above cuts. We compare it to the theoretical distribution which is the same for the two angles. We see that the cuts have limited effect on $\cos \theta_{1}$, but the rate for $\cos \theta_{2} \sim \pm 1$ is suppressed. This is because in that configuration, one of the leptons is nearly aligned with the boost direction needed to go to the lab frame from the $Z_{2}$ rest frame, and thus performing that boost will reduce its energy and make it less likely to pass the $p_{T}$ cut. This effect is small for $\cos \theta_{1}$ because the lepton energies in the $Z_{1}$ rest frame are much larger.

In Fig. 3.3, comparing the blue (solid) curve to the light-blue (dot-dashed) histogram, we see that the experimental cuts reduce the event rate for small $M_{2}$. Even after these cuts, 


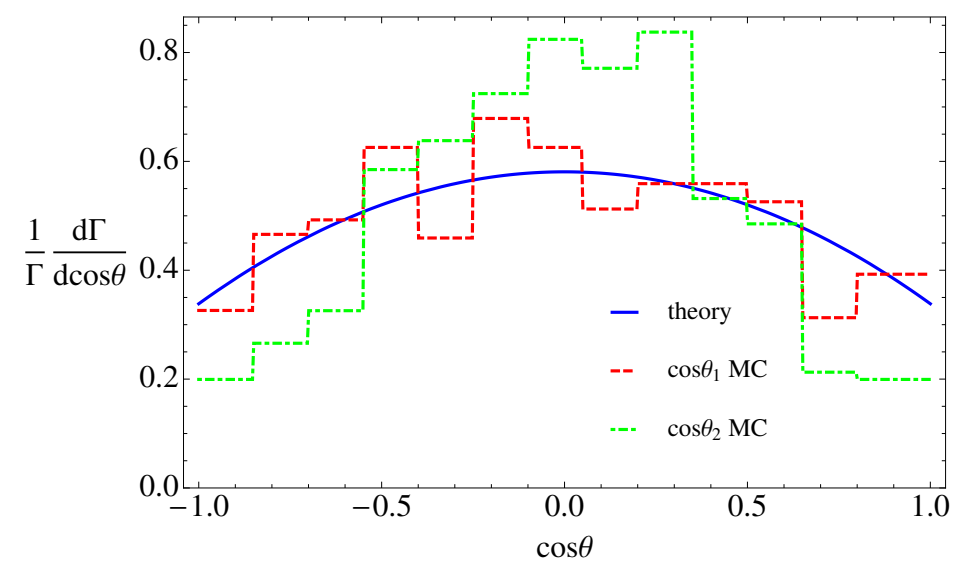

Figure 3.2. Normalized distribution for $\cos \theta$ in the $a_{h}$ scenario. The blue (solid) curve is the same as the theory curve from Fig. 3.1, the red (dashed) histogram is the distribution for $\cos \theta_{1}$ for 1000 Monte Carlo events, while the green (dot-dashed) histogram is $\cos \theta_{2}$ for the same events.

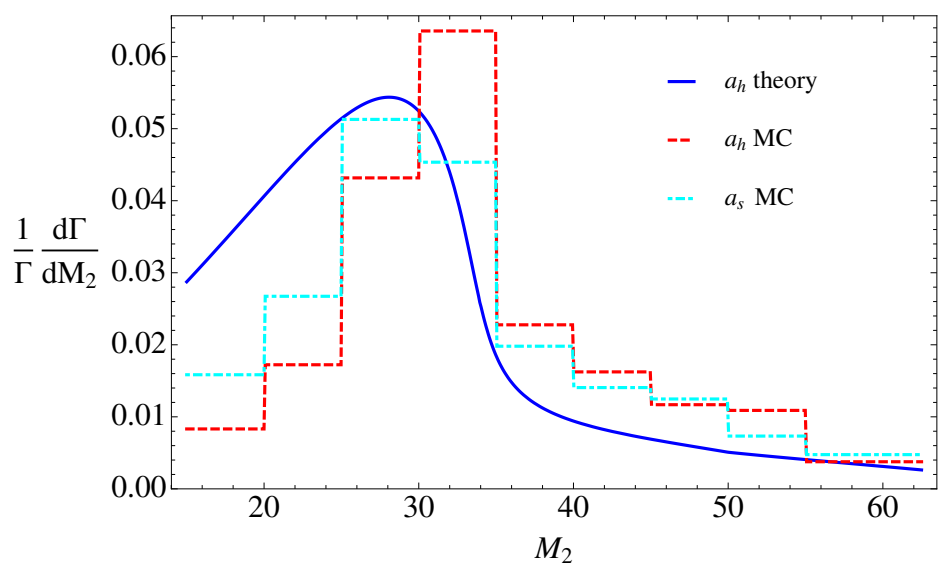

Figure 3.3. Normalized $M_{2}$ distributions. The blue (solid) curve is the theory prediction in the $a_{h}$ scenario, while the light blue (dot-dashed) histogram is 1000 Monte Carlo events also in the $a_{h}$ scenario. The red (dashed) histogram is 1000 events in the $a_{s}$ scenario.

however, the histograms for $a_{h}$ and $a_{s}$ still differ, so the experimental cuts do not wash out the discriminating power. 


\subsection{Distinguishing Operators}

In order to estimate the ability of the LHC to discriminate a Higgs-like scenario dominated by $a_{h}$ from other scenarios, we employ a likelihood analysis of the generated events. We consider only signal events because requiring the invariant mass of the four lepton system to be near the mass of the new boson can make the signal to background ratio significantly larger than one. Furthermore, reweighing techniques such as the one laid out in [48, can be used to further purify the event selection.

We use a standard unbinned likelihood analysis which is described in detail in [9]. We can use the computed normalized differential cross section as a probability distribution $P\left(\Phi, \theta_{i}, M_{i} \mid a_{i}\right)$

for each operator $a_{h}, a_{s}$, and $a_{Z \gamma}$. The normalization is computed with the $M_{i}$ cuts described above because they are independent of Lorentz frame. Taking the $p_{T}$ and $\eta$ acceptance into account in $P$ would improve the statistical power of the test, but because those cuts are framedependent, we leave that to further work.

Given a sample of $N$ events, we can then construct a likelihood $\mathcal{L}\left(a_{i}\right)=\prod_{j=1}^{N} P_{j}\left(a_{i}\right)$. With this likelihood we can then compare two different scenarios, $a_{1}$ and $a_{2}$ by constructing a hypothesis test with test statistic defined by [49]

$$
\Lambda=2 \log \left[\mathcal{L}\left(a_{1}\right) / \mathcal{L}\left(a_{2}\right)\right]
$$

Since we are taking the resonance mass as input and using the normalized differential cross sections to construct our likelihood functions, there are no free parameters (nuisance parameters) in this ratio, making this a simple hypothesis test.

To estimate the expected significance of discriminating between two different hypotheses corresponding to two different operators, we follow a similar analysis to that found in [9]. To begin, we take one hypothesis as true, say $a_{1}$ and generate a fixed number $N$ of $a_{1}$ events. We 


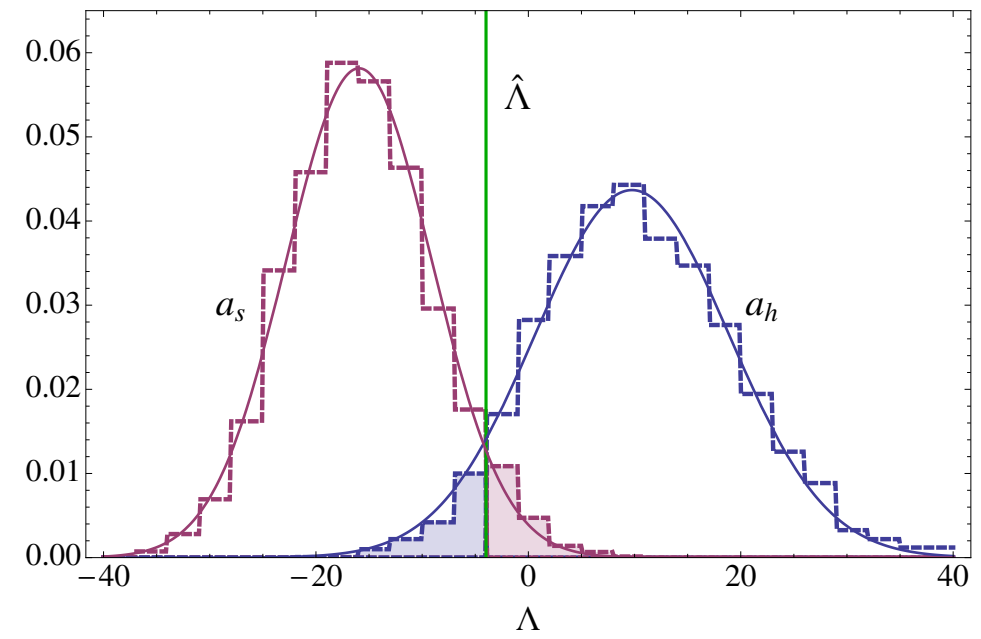

Figure 3.4. Normalized distribution of our test statistic $\Lambda$ when $a_{h}$ is true on the right (blue), and when $a_{s}$ is true on the left (pink). Each histogram is the result of 5000 pseudo-experiments with 50 events each. The vertical (green) line is $\hat{\Lambda}$ defined in Eq. (3.4) such that the area to the right of $\hat{\Lambda}$ under the $a_{s}$ histogram is equal to the area to the left of $\hat{\Lambda}$ under the $a_{h}$ histogram. We also draw a Gaussian over each histogram with the same median and standard deviation.

then construct $\Lambda$ as above for a large number of pseudo-experiments each containing $N$ events in order to obtain a distribution for $\Lambda$. We then repeat this exercise taking $a_{2}$ to be true and again obtain a distribution for $\Lambda$. These two distributions are shown in Fig. 3.4 comparing $a_{h}$ and $a_{s}$. This figure shows 5000 pseudo-experiments of 50 events each, which shows a clear separation between the two scenarios.

With the two distributions for $\Lambda$ in hand we can compute an approximate significance by the following procedure. If we denote the distribution with negative mean as $f$ and the distribution with positive mean as $g$, we find a value $\hat{\Lambda}$ such that

$$
\int_{\hat{\Lambda}}^{\infty} f d x=\int_{-\infty}^{\hat{\Lambda}} g d x .
$$

Schematically, this value of $\hat{\Lambda}$ corresponds to a value such that if the experiment observed that value for the test statistic, it would have no discriminatory power between the two scenarios. 
We then interpret the probability given by either side of Eq. (3.4) as a one sided Gaussian probability, which can then be interpreted in terms of number of $\sigma$. This procedure is shown schematically in Fig. 3.4 with the areas of the two shaded regions being equal and corresponding to the probability of excluding the correct hypothesis. For a simple hypothesis test, this Gaussian approximation is often sufficient [49], and we see from Fig. 3.4 that the $\Lambda$ distributions are well approximated by Gaussians.

This procedure is repeated many times for a range of numbers of events $N$ to obtain a significance as a function of $N$ for each hypothesis. We show this for the case where $a_{1}=a_{h}$ and $a_{2}=a_{s}$ or $a_{2}=a_{Z \gamma}$ in Fig. 3.5. We see that with $\mathcal{O}(50)$ events, we can distinguish renormalizable from nonrenormalizable coupling to $Z Z$ at $95 \%$ confidence, and with $\mathcal{O}(100)$ events we can get a $99 \%$ exclusion. The operator $a_{Z \gamma}$ can be distinguished from $a_{h}$ at $95 \%$ confidence with as few as 20 events. The third possibility, which we do not show, is even easier; $a_{s}$ and $a_{Z \gamma}$ can be distinguished from one another at $95 \%$ with just 10 events.

\subsection{Conclusions and Outlook}

Testing the properties of the newly discovered resonance near $125 \mathrm{GeV}$ is of utmost importance. While the rate and branching ratio data are consistent with the new particle being the Standard Model Higgs, direct tests of its properties are still essential. In this chapter we have examined the discriminating power of events where the new particle decays to four leptons. These events can be used to measure the Lorentz transformation properties of this particle, but even if it is confirmed to be a parity even scalar, it still need not be the Higgs; it could couple to the gauge bosons via higher dimensional operators rather than via the renormalizable operator in the Standard Model.

We have analyzed how well kinematic distributions in four lepton events can distinguish between different tensor structures of the coupling to gauge bosons. In particular, we looked a 

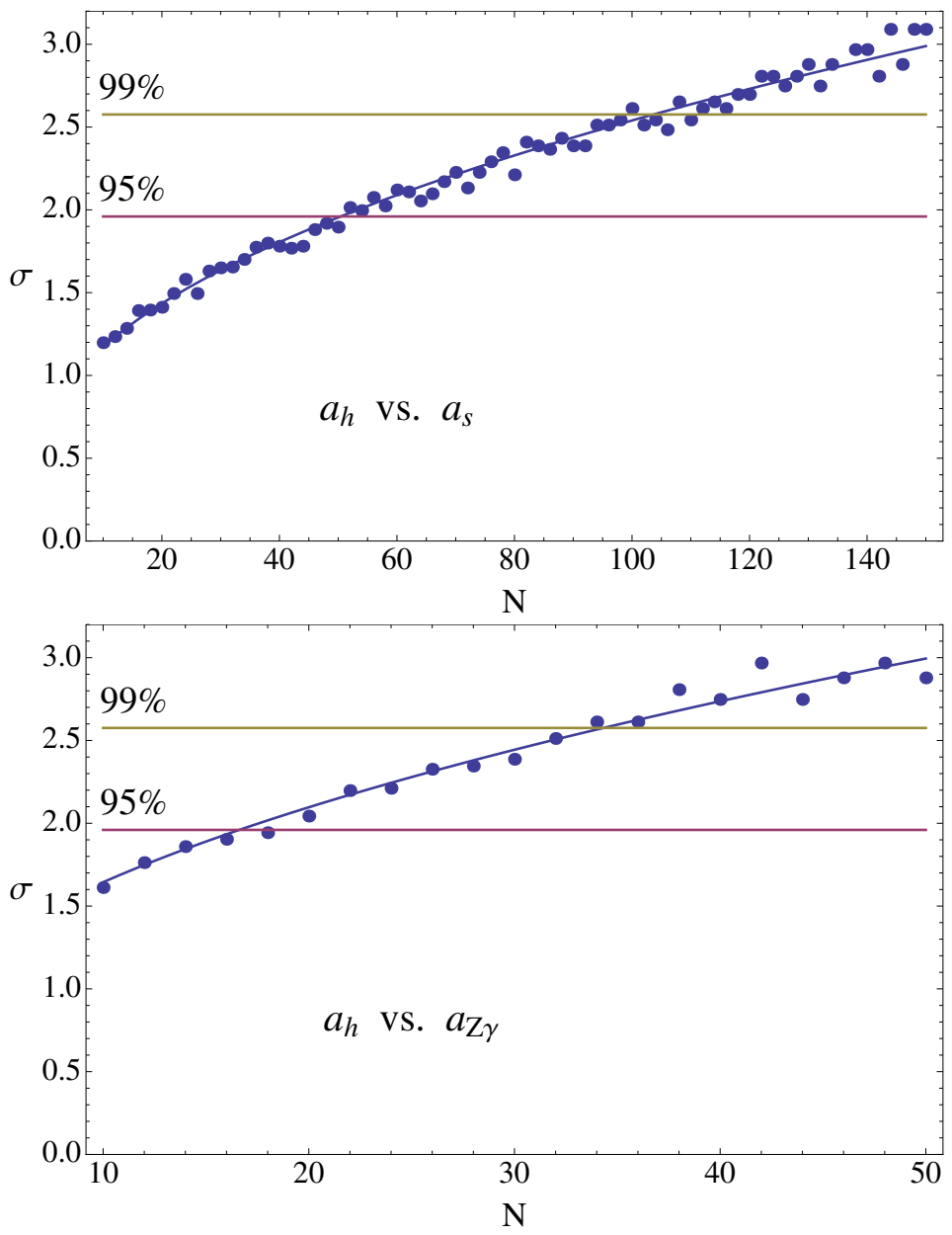

Figure 3.5. Expected significance as a function of number of events in the case of $a_{h}$ vs $a_{s}$ on top, and $a_{h}$ vs $a_{Z \gamma}$ on bottom. We use a different horizontal scale for the top and bottom plots because far fewer events are needed to discriminate $a_{h}$ from $a_{Z \gamma}$ than from $a_{s}$. We also fit with a function proportional to $\sqrt{N}$, which is the expected scaling. We mark the $\sigma$ value of $95 \%$ and $99 \%$ confidence level exclusion.

coupling directly to $Z_{\mu} Z^{\mu}$, as well as couplings to a pair of field strength tensors of the $Z$, and a coupling to the field strength of the $Z$ and of the photon. All three scenarios will produce one lepton pair near the $Z$ pole, while the other pair will have much lower invariant mass. We find that with $\mathcal{O}(50)$ signal events, a Higgs-like state can be discriminated from $Z Z$ field strength tensor couplings with $95 \%$ confidence, while only 20 events are needed to make the 
same determination for field strength coupling to $Z \gamma$. This shows that the LHC has excellent prospects to constrain the tensor structure of the new state's coupling to gauge bosons. 


\section{CHAPTER 4}

\section{Dark Matter and Vector-like Leptons From Gauged Lepton Number}

\subsection{Introduction}

With the recent discovery of a new resonance with standard model (SM) Higgs like properties [1, 2] the final piece of the SM appears to be in place. It is well known, however, that there are questions for which the SM has no answer and beyond the standard (BSM) physics is needed. Chief among these questions is the nature of dark matter (DM) and the mechanism which makes it stable. It is also well known that the renormalizable SM Lagrangian possesses an (anomalous) accidental global symmetry associated with the conservation of overall lepton number. If one allows for higher dimensional operators, lepton violating interactions can occur at dimension five, but to date no such processes (with the possible ambiguous exception of neutrino masses) have been observed experimentally [50]. This is perhaps an indication that lepton number is a more fundamental symmetry which prevents the generation of SM lepton number violating operators. In this work, we connect the apparent lack of lepton number violation to the stability of thermal relic dark matter, by deriving both from a $U(1)_{L}$ gauge symmetry associated with lepton number.

Gauging lepton number is attractive for both phenomenological as well as theoretical reasons and the possibility of lepton number (and also baryon number) as a local gauge symmetry was first explored in [51, 52]. However, the first complete and consistent model of gauged lepton number (and baryon number) was not explored until more recently in [53] with numerous 
variations following [54 59]. Here we explore a particular realization where the DM arises as part of the exotic lepton sector required by gauging lepton number and the attendant need to cancel anomalies.

The DM candidate is a Dirac electroweak (mostly) singlet neutrino stabilized by an accidental global symmetry of the renormalizable Lagrangian which is preserved even after lepton number is spontaneously broken. As we will see, as a byproduct of the lepton breaking mechanism and the requirement of a viable DM candidate, one also obtains a set of vector-like leptons which can have interesting phenomenology at the LHC through either direct production or through modifications of Higgs decays to SM particles.

We extend the SM gauge group to $S U(3)_{c} \otimes S U(2)_{W} \otimes U(1)_{Y} \otimes U(1)_{L}$ where the SM leptons are charged under $U(1)_{L}$. The anomalous $U(1)_{L}$ requires us to add a new set of leptons with the appropriate quantum numbers to cancel anomalies. Typically, $U(1)_{L}$ is spontaneously broken by the vacuum expectation value of a SM singlet scalar in such a way that Majorana masses can be generated for the right-handed neutrinos, (whose presence is required by anomaly cancellation [53]). Such constructions allow for a simple realization of the well known 'seesaw' mechanism of neutrino mass generation, but do not contain viable dark matter candidates without additional assumptions or particle content.

Here, motived by the desire for a thermal DM candidate, we choose to break lepton number with a SM singlet scalar carrying $L=3$. This leads to a remnant global $U(1)$ symmetry preventing decay of the lightest new lepton which stabilizes the DM candidate. This global symmetry is a consequence of the gauge symmetry and particle content of the model and does not need to be additionally imposed. It also ensures that the model is safe from dangerous flavor violating processes which are highly constrained by experiment. An automatic consequence of this construction is that one also obtains a new generation of vector-like (with respect to the SM) leptons after the spontaneous breaking of lepton number. This type of lepton spectrum has 
garnered recent interest in the context of modifications to the Higgs decay into diphotons [60 66 and was also recently shown to be useful for baryogenesis [67, 68].

The organization of this papers is as follows. In Sec. 4.2 we briefly review the gauging of lepton number and cancellation of anomalies. We also discuss the details of the lepton breaking mechanism as well as the particle content and Lagrangian. In Sec. 4.3 we discuss the DM candidate and stability and obtain the relic abundance for a range of DM masses. We also examine the direct and indirect detection prospects. In Sec. 4.4 we discuss constraints as well as LHC phenomenology and examine the effect of the vector-like leptons on the Higgs to diphoton rate. We present our conclusions and an overview of possible future work in Sec. 4.5 .

\subsection{The Model}

The SM gauge group is extended to $S U(3)_{c} \otimes S U(2)_{W} \otimes U(1)_{Y} \otimes U(1)_{L}$ where $L$ represents the lepton charge. We restrict ourselves to the minimal particle content consisting of a set of anomaly-canceling exotic leptons, plus the new gauge field and a SM singlet scalar which breaks lepton number spontaneously. In principle, this theory is UV-complete up to large energies, and we restrict ourselves to considering renormalizable interactions. We discuss each of these ingredients, including the interactions, below.

\subsubsection{Anomaly Cancellation}

The anomalies introduced when gauging lepton number and various ways to cancel them with the addition of new fermions are discussed in detail in [53 55]. All options include three generations of right-handed singlet neutrinos $\left(\nu_{R i}\right.$, considered as part of the SM) with quantum numbers $\nu_{R i} \equiv(1,0,1)$ under $\left(S U(2)_{W}, U(1)_{Y}, U(1)_{L}\right)$ and $i=e, \mu, \tau$. We define all SM leptons to have $L=1$. In addition to $\nu_{R i}$, one must add new electroweak doublet and singlet leptons to cancel the gauge anomalies. There are several options; here we focus on a simple construction making 
use of two exotic generations of chiral fermions which together form a vector-like set under the SM gauge group [55], ensuring that anomaly cancellation in the SM gauge factors is preserved. The first set of new fermions is a sequential fourth generation of leptons carrying lepton number $L=L^{\prime}$,

$$
\begin{aligned}
& \ell_{L}^{\prime} \equiv\left(\nu_{L}^{\prime} e_{L}^{\prime}\right) \equiv\left(2,-1 / 2, L^{\prime}\right), \\
& e_{R}^{\prime} \equiv\left(1,-1, L^{\prime}\right), \nu_{R}^{\prime} \equiv\left(1,0, L^{\prime}\right) .
\end{aligned}
$$

The second is a mirror set of opposite chirality with lepton number $L=L^{\prime \prime}=L^{\prime}+3$,

$$
\begin{aligned}
& \ell_{R}^{\prime \prime} \equiv\left(\nu_{R}^{\prime \prime} e_{R}^{\prime \prime}\right) \equiv\left(2,-1 / 2, L^{\prime \prime}\right), \\
& e_{L}^{\prime \prime} \equiv\left(1,-1, L^{\prime \prime}\right), \nu_{L}^{\prime \prime} \equiv\left(1,0, L^{\prime \prime}\right),
\end{aligned}
$$

where the condition,

$$
L^{\prime}-L^{\prime \prime}=-3
$$

is required by anomaly cancellation. The addition of two sets of chiral fermions carrying lepton number which together form a vector-like set under the SM also avoids the need to add new quarks to cancel anomalies, although scenarios with exotic quarks are also interesting and have been explored in the context of gauged baryon number [53 55]. The particle content in Eqs.4.1) and 4.2 is similar to that obtained in [57] where baryon number is also gauged and one obtains a vector-like set of 'lepto-quarks' as well as a potential DM candidate. Here we focus on only gauging lepton number which requires a simpler scalar sector and fewer new particles. 


\subsubsection{Gauge and Higgs Sector}

The gauging of lepton number will introduce a new spin-1 vector boson which we label $Z_{L}$. In addition to the usual Abelian vector field kinetic terms, the $U(1)_{L}$ gauge field will have interactions,

$$
\begin{aligned}
& \mathcal{L} \supset\left(D^{\mu} \Phi\right)^{\dagger}\left(D_{\mu} \Phi\right)+\frac{\epsilon}{2} Z_{L}^{\mu \nu} B_{\mu \nu} \\
& +\bar{l}_{L}^{\prime} D_{\mu} \gamma^{\mu} l_{L}^{\prime}+\bar{l}_{R}^{\prime \prime} D_{\mu} \gamma^{\mu} l_{R}^{\prime \prime}+\bar{l}_{i} D_{\mu} \gamma^{\mu} l_{i},
\end{aligned}
$$

where $D^{\mu}=\partial^{\mu}+i g^{\prime} L Z_{L}^{\mu}$ with $L$ the lepton number assignment for a particular field. $\Phi \equiv$ $\left(1,0, L_{\Phi}\right)$ is the SM singlet scalar carrying lepton number whose vev $\left(v_{\phi}\right)$ breaks the $U(1)_{L}$ spontaneously. The index $i=e, \mu, \tau$ runs over all SM leptons while $l=\ell, e, \nu$ where $\ell$ is an $S U(2)$ doublet and $e, \nu$ are singlets. Note there is no $\delta M^{2} Z_{L \mu} Z^{\mu}$ term since $\Phi$ is not charged under the SM and the Higgs does not carry $L$.

The parameter $\epsilon$ encapsulates the degree of kinetic mixing between $U(1)_{L}$ and $U(1)_{Y}$. One can in principle impose $\epsilon=0$ at tree level through symmetries, but in general it is a free parameter of the theory and is additively renormalized by loops of leptons. While any value of $\epsilon$ at the weak scale can be engineered, the loop-induced piece is typically of order $10^{-3}$, small enough to be consistent with experimental constraints without undue fine tuning.

After lepton and electroweak symmetry breaking $\epsilon$ also leads to $Z-Z_{L}$ mixing parameterized by [69],

$$
\tan 2 \xi=\frac{2 M_{Z}^{2} s_{W} \epsilon \sqrt{1-\epsilon^{2}}}{M_{Z_{L}}^{2}-M_{Z}^{2}\left(1-\epsilon^{2}\right)+M_{Z}^{2} s_{W}^{2} \epsilon^{2}},
$$

where $\xi$ is the $Z_{L}-Z$ mixing angle and $M_{Z}, M_{Z_{L}}$ are the masses. In the absence of mixing, $M_{Z_{L}}=L_{\Phi} g^{\prime} v_{\phi}$. As we will see, since this mixing is constrained to be small by direct searches for dark matter (with weaker constraints from precision measurements [69 71]) we take $M_{Z}, M_{Z_{L}}$ as the physical masses as well. 
In the Higgs sector the existence of $\Phi$ allows for an expanded scalar potential,

$$
\begin{aligned}
& V(H, \Phi)=-\mu_{H}^{2} H^{\dagger} H+\lambda_{H}\left|H^{\dagger} H\right|^{2} \\
& -\mu_{\Phi}^{2} \Phi^{\dagger} \Phi+\lambda_{\Phi}\left|\Phi^{\dagger} \Phi\right|^{2}+\lambda_{h p} \Phi^{\dagger} \Phi H^{\dagger} H
\end{aligned}
$$

where $H \equiv(2,-1 / 2,0)$ is the SM Higgs doublet. Once lepton number is broken, the real component of $\Phi$ obtains a vacuum expectation value $\langle\Phi\rangle=v_{\phi} / \sqrt{2}$, while the Higgs boson $H$ obtains its own vev, $\langle H\rangle=\left(0, v_{h} / \sqrt{2}\right)$ to break the electroweak symmetry. The scale $v_{\phi}$ will be the only new dimensional scale introduced, with all of the other parameters being dimensionless couplings. We will see below in Sec. 4.2 .4 that $L_{\Phi}=3$ is preferred.

The presence of the 'Higgs portal' coupling $\lambda_{h p}$ will generically lead to mixing between the real singlet components of $\Phi$ and $H$ parameterized by the mixing angle,

$$
\tan 2 \theta=\frac{\lambda_{h p} v_{h} v_{\phi}}{\lambda_{\Phi} v_{\phi}^{2}-\lambda_{H} v_{h}^{2}} .
$$

This mixing leads to the mass eigenstates,

$$
\begin{aligned}
h & =c_{\theta} h_{o}-s_{\theta} \phi_{o}, \\
\phi & =s_{\theta} h_{o}+c_{\theta} \phi_{o},
\end{aligned}
$$

where $\phi_{o}$ and $h_{o}$ are the gauge eigenstates and $\phi, h$ are the mass eigenstates with masses,

$$
m_{h, \phi}^{2}=\left(\lambda_{H} v_{h}^{2}+\lambda_{\Phi} v_{\phi}^{2}\right) \mp \sqrt{\left(\lambda_{\Phi} v_{\phi}^{2}-\lambda_{H} v_{h}^{2}\right)^{2}+\lambda_{h p}^{2} v_{h}^{2} v_{\phi}^{2}}
$$

where we have assumed $m_{\phi}>m_{h}$ and defined $c_{\theta}=\cos \theta, s_{\theta}=\sin \theta$, etc. The coupling $\lambda_{h p}$ will also lead to a tree level shift in the Higgs quartic coupling [72], which provides a mechanism for stabilizing the vacuum in the presence of the exotic charged leptons with large Yukawa 
couplings to the SM Higgs. It was shown to be a particularly efficient stabilization mechanism when $m_{\phi} \gg m_{h}$, even for small mixing angles [62].

\subsubsection{Global Symmetries and Breaking L}

The two new sets of leptons along with the SM lepton sector comprise three separate sectors labeled by their lepton number $L=1, L^{\prime}, L^{\prime \prime}$ for which global $U(1)$ symmetries can be associated. These global symmetries are each separately conserved by the SM and $U(1)_{L}$ interactions. Yukawa interactions (assuming $L_{\Phi}$ permits them) will break these symmetries in realistic models, as discussed below. A combination of precision electroweak, collider, and direct detection constraints prohibit a stable lepton which carries electroweak charge. Thus, couplings to the Higgs must not be too large and the DM can not receive its mass solely from the SM Higgs, leading to the need to generate an additional contribution to the DM mass which does not come from electroweak symmetry breaking.

From these considerations one concludes that the SM singlets $\nu_{R}^{\prime}$ and $\nu_{L}^{\prime \prime}$ or some combination must compose the majority of the DM. Majorana masses can be generated by choosing the lepton breaking scalar to carry $L_{\Phi}=2 L^{\prime}$ or $L_{\Phi}=2 L^{\prime \prime}$. However, this choice still leaves either $L^{\prime}$ or $L^{\prime \prime}$ unbroken meaning that the lightest lepton of the corresponding sector will be stable and only receive its mass from its couplings to the Higgs, which as discussed is ruled out by experiment. It is clear that in order to avoid a heavy stable lepton with unacceptably large couplings to the $Z$ or Higgs boson one must choose $L_{\Phi}$ such that it generates an interaction between the $L^{\prime}$ and $L^{\prime \prime}$ sectors. The anomaly cancellation condition of Eq. (4.3) ensures that the only possibility is $L_{\Phi}=3$. 


\subsubsection{Yukawa Sector}

Given $L_{\Phi}=3$, the Lagrangian for the Yukawa sector of the new leptons can be written,

$$
\begin{aligned}
& \mathcal{L} \supset-c_{\ell} \Phi \bar{\ell}_{R}^{\prime \prime} \ell_{L}^{\prime}-c_{e} \Phi \bar{e}_{L}^{\prime \prime} e_{R}^{\prime}-c_{\nu} \Phi \bar{\nu}_{L}^{\prime \prime} \nu_{R}^{\prime}-y_{e}^{\prime} H \bar{\ell}_{L}^{\prime} e_{R}^{\prime} \\
& -y_{e}^{\prime \prime} H \bar{\ell}_{R}^{\prime \prime} e_{L}^{\prime \prime}-y_{\nu}^{\prime} \tilde{H} \bar{\ell}_{L}^{\prime} \nu_{R}^{\prime}-y_{\nu}^{\prime \prime} \tilde{H} \bar{\ell}_{R}^{\prime \prime} \nu_{L}^{\prime \prime}+h . c .
\end{aligned}
$$

In general these couplings are complex, containing phases which can lead to CP violation, but for simplicity we assume all couplings in Eq. 4.10) are real (but see [73, 74] for recent studies of $\mathrm{CP}$ violating effects on the diphoton rate coming from vector-like leptons). It is also clear from Eq.4.10 that once $\Phi$ obtains a vev the couplings $c_{\ell}, c_{e}$, and $c_{\nu}$ will lead to vector-like (with respect to the $\mathrm{SM}$ ) masses for the exotic leptons. The new leptons will also receive mass contributions from electroweak symmetry breaking through the $y_{\nu, e}^{\prime}, y_{\nu, e}^{\prime \prime}$ couplings. Note also that unless $L^{\prime}, L^{\prime \prime}=0$, explicit Majorana masses for $\nu_{R}^{\prime}$ and $\nu_{L}^{\prime \prime}$ are not allowed nor will they be generated after lepton number breaking unless $L^{\prime}=-L^{\prime \prime}=-3 / 2$ (This case was considered explicitly in the context of gauged lepton and baryon number with vector-like 'lepto-quarks' [57]). We avoid these choices in what follows.

In principle there may still be couplings between the exotic and SM leptons. Since we have taken SM lepton number to be $L=1$, this implies that $L^{\prime}, L^{\prime \prime} \neq 1$ in order to avoid mixing with SM leptons which can lead to dangerous flavor changing neutral currents as well as the decay of the DM. If we choose $L^{\prime}=-4$, which fixes $L^{\prime \prime}=-1$ then, in addition to those in Eq.4.10, one can also generate interactions between the SM and the new lepton sector given by,

$$
\mathcal{L} \supset y \Phi \bar{\nu}_{R}^{c \prime} \nu_{R i}+h . c .
$$

Once $\Phi$ obtains a vev, this will lead to mixing between the SM right-handed neutrinos, $\nu_{R i}$ and the exotic right handed neutrino, $\nu_{R}^{\prime}$. This also implies that the exotic lepton sector can decay 
to the SM, thus eliminating this scenario as an explanation for dark matter. To summarize, in order to avoid mixing with the SM and ensure a stable DM candidate, we take $\left(L^{\prime}, L^{\prime \prime}\right) \neq$ $(1,4),(-4,-1),(-2,1)$. Furthermore, to avoid Majorana mass terms we also assume $\left(L^{\prime}, L^{\prime \prime}\right) \neq$ $(0,3),\left(-\frac{3}{2}, \frac{3}{2}\right),(-3,0)$. Thus our complete Yukawa sector Lagrangian is given by Eq. 4.10 and $L^{\prime}$ can otherwise be any real number satisfying $L^{\prime}=-3+L^{\prime \prime}$.

In the limit that the Yukawa couplings $c_{i} \rightarrow 0$, one recovers the global symmetries which separately preserve $L^{\prime}, L^{\prime \prime}$ and $L_{S M}$. As a result, $c_{i} \ll 1$ are technically natural, implying that vector-like masses for the new leptons much smaller than $v_{\phi}$ are natural. We also note that small values of the $y_{\nu, e}^{\prime}, y_{\nu, e}^{\prime \prime}$, and $y_{\nu i}^{S M}$ Higgs Yukawa couplings are technically natural.

It is worth noting that Eq. 4.10 is very similar to the Yukawa sectors proposed in a generic framework in [60, 62], but here arises from $U(1)_{L}$ gauge invariance and anomaly cancellation. Only one new scale $\left(v_{\phi}\right)$ is introduced, with the masses of the new fermions following from dimensionless couplings. Furthermore, the global symmetries needed to protect against dangerous mixing with SM leptons and insuring the existence of a stable DM particle are guaranteed by $U(1)_{L}$ gauge invariance as opposed to being imposed by hand.

\subsubsection{Experimental Constraints}

Low energy experiments place a limit on the parameters which describe the $Z_{L}$ sector. Since the SM Higgs does not carry lepton number and $\Phi$ is a SM singlet, there is no mass-mixing between $Z_{L}$ and the SM electroweak interaction at tree level. Furthermore since $Z_{L}$ does not couple to quarks, direct search limits from the LHC are rather weak, and the strongest limits are obtained from constraints on four-lepton operators derived from LEP II data [75]; these require

$$
v_{\phi} \geq 1.7 \mathrm{TeV}
$$

roughly independently of the value of $g^{\prime}$. 


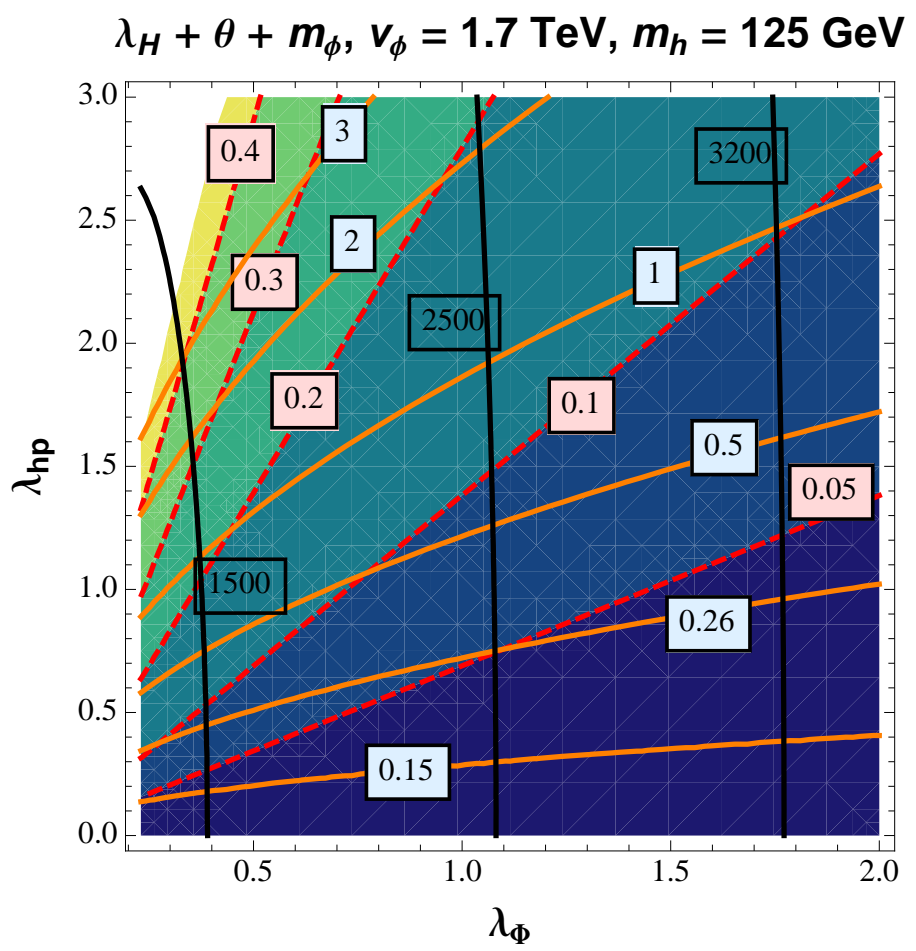

Figure 4.1. Contours of Higgs mixing angle $\theta$ (red-dotted), Higgs quartic coupling $\lambda_{H}$ (orange-solid), and heavy scalar mass $m_{\phi}$ in $\mathrm{GeV}$ (black-solid) as defined in Eqs.(4.7) Eq.(4.9) as a function of scalar couplings $\left(\lambda_{h p}, \lambda_{\Phi}\right)$ in Eq. (4.6).

This lower bound and the experimentally measured value of $m_{h} \simeq 125 \mathrm{GeV}$ constrains the quartic couplings in the scalar potential of Eq. (4.6) through Eq. (4.7) and (4.9). Fixing $v_{\phi}=1.7 \mathrm{TeV}$ and $m_{h}=125 \mathrm{GeV}$ we can then examine the scalar mixing angle $\theta$, the Higgs quartic $\lambda_{H}$, and the heavy scalar mass eigenstate $m_{\phi}$ as functions of the scalar couplings $\lambda_{h p}$ and $\lambda_{\Phi}$. In Fig 4.1 we show contours of $\lambda_{H}\left(\lambda_{\Phi}, \lambda_{h p}\right)$ (solid-orange), $\theta\left(\lambda_{\Phi}, \lambda_{h p}\right)$ (dotted-red), and $m_{\phi}\left(\lambda_{\Phi}, \lambda_{h p}\right)$ (solid-black) in the $\lambda_{h p}-\lambda_{\Phi}$ plane. As can be seen, values of $\theta \lesssim 0.1-0.2$ can be obtained for quartic couplings of $\mathcal{O}(1)$ and heavy scalar masses $\sim 2.5 \mathrm{TeV}$. To obtain mixings as large as $\theta \sim 0.4$ requires $\lambda_{H} \sim 3$ and small $\lambda_{\Phi} \lesssim 0.5$ with $m_{\phi} \sim 1.5 \mathrm{TeV}$. In general we find $m_{\phi} \gtrsim 1 \mathrm{TeV}$ for $v_{\phi}=1.7 \mathrm{TeV}$, possibly within reach of the LHC, but more likely too heavy to be produced directly. 


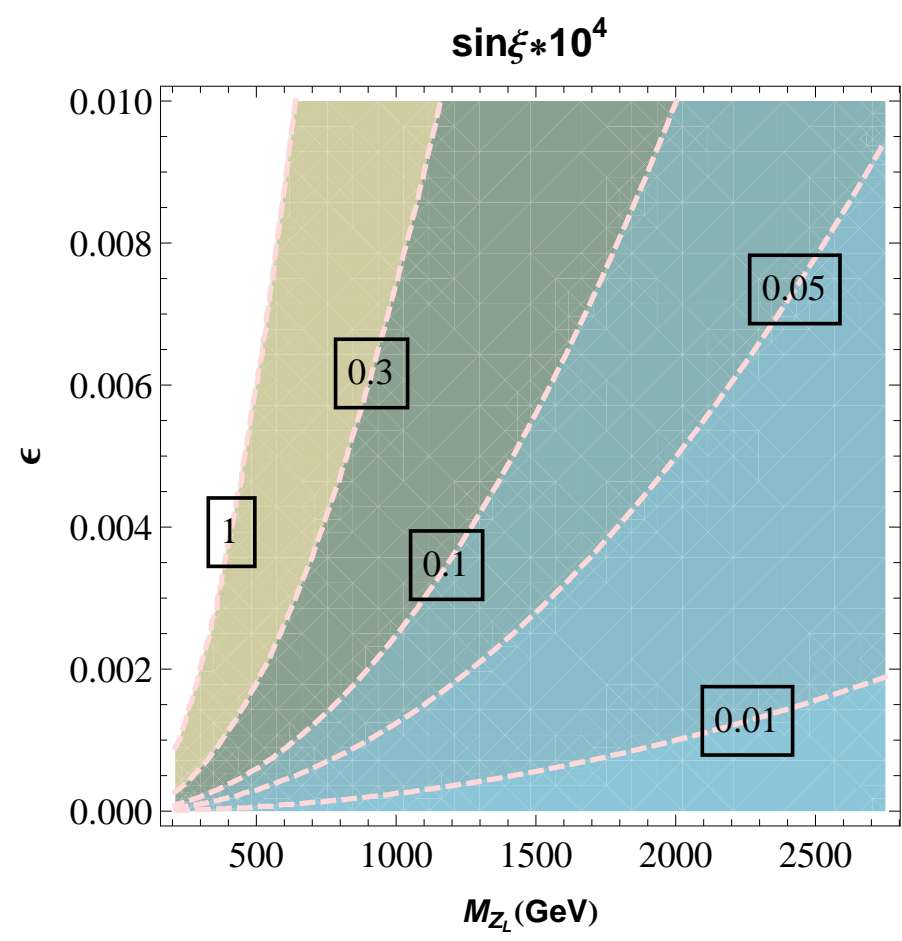

Figure 4.2. Contours of the $Z-Z_{L}$ mixing angle $\sin \xi\left(\times 10^{4}\right)$ in the $\epsilon-M_{Z_{L}}$ plane (see Eq.4.5).

Precision measurements on the $Z$-pole also constrain the degree of $Z_{L^{-}} Z$ mass mixing. Since this occurs at loop level (through loops of the SM and exotic leptons as well as scalars), it will typically be small enough $\left(\lesssim 10^{-3}\right)$ for any $v_{\phi}$ consistent with the LEP II bound. There are also constraints (via $\sin \xi$ in Eq. 4.5p) on the kinetic mixing parameter from direct detection [76]), which are comparable to the expected size induced by loops of leptons. Using Eq.4.5 we examine the $\epsilon-M_{Z_{L}}$ parameter space for typically allowed values of $\sin \xi \lesssim 10^{-4}$ over a range of $Z_{L}$ masses. In Fig 4.2 we present contours of $\sin \xi \times 10^{4}$ in the $\epsilon-M_{Z_{L}}$ plane for small values of the kinetic mixing parameter $\epsilon$ as would be favored in theories where $\epsilon=0$ at tree level as discussed in Sec 4.2.2. We can see that for $M_{Z_{L}} \sim 1 \mathrm{TeV}$ one can obtain a $Z-Z_{L}$ mixing angle of $\sin \xi \sim 0.1 \times 10^{-4}$ with a kinetic mixing of $\epsilon \sim 0.002$. 


\subsubsection{Possible Extensions}

There are a number of possibilities for how one could extend this model or embed it into a more complete theory. For instance,with the need to break lepton number spontaneously, the question as to how one obtains $v_{\phi}$ naturally also arises. One could imagine embedding this model in a supersymmetric version as was done in [55, 56, 77] for other gauged lepton number constructions. Another possibility is to have the scalar sector of this model arise as part of a set of goldstone bosons resulting from a strongly broken global symmetry as for example [78].

Another possibility for generating natural values for not only $v_{\phi}$, but also the electroweak scale $\left(v_{h}\right)$ is through dimensional transmutation where $v_{\phi}$ is generated radiatively [79]. This scale is then inherited by the SM through the 'Higgs Portal' as done recently in [80] for a hidden $U(1)$ gauge extension of the SM, but we leave it to a future study to explore this possibility. For the remainder of this study we simply set $v_{\phi}$ to its lower bound of $v_{\phi}=1.7 \mathrm{TeV}$.

One can also extend the theory to obtain $\epsilon=0$ at tree level in Eq.(4.4) by positing that the $U(1)_{L}$ gauge symmetry arises out of a larger non-Abelian gauge symmetry which forbids $\epsilon \neq 0$ [81] and is broken at some high scale $\Lambda$ down to $U(1)_{L}$. Below the scale $\Lambda$, but above the lepton and electroweak breaking scales, loop corrections due to hyper-charged leptons vanish provided the leptons satisfy an orthogonality condition [81,

$$
\operatorname{Tr}(L Y)=0 .
$$

Combined with the anomaly cancellation constraint in Eq. 4.3 , this would determine the exotic lepton numbers to be $L^{\prime}=-3$ and $L^{\prime \prime}=0$. Below $v_{\phi}$ and $v_{h}$ there will be loop induced (from both leptons and scalars) corrections which generate a kinetic mixing, but typically $\epsilon \ll 1$.

Note, that although we have only gauged lepton number, this is enough to prevent the dimension six operators of the form $\mathcal{L} \sim \frac{1}{\Lambda^{2}} q q q \ell$ (for appropriate lepton number assignment to 
the lepton breaking scalar) which might lead to proton decay. However, while baryon number violating operators at dimension six are forbidden, higher order operators are still allowed since baryon number is not protected by a gauge symmetry. The leading operator that might mediate

proton decay, $\frac{1}{\Lambda^{8}}(q q q \ell)(\ell H)^{2} \Phi^{\dagger}$, first occurs at dimension twelve while $\Delta B=2$ operators with $\Delta L=0$ are allowed at dimension 9 [82], as in the SM. For scales $\Lambda \gtrsim \mathcal{O}(100) \mathrm{TeV}$ the model considered here should be reasonably safe from the effects of these potentially dangerous operators. Of course one can extend this model to include gauged baryon number as well to prevent these operators [57].

Finally is is worth mentioning that this model possesses many ingredients which may be helpful for explaining the baryon asymmetry of the universe. The current construction automatically contains new massive states as well as new interactions containing $C P$-violating phases. It would be interesting to explore whether or not it is capable of explaining this asymmetry as well as dark matter. Since the WIMP in this theory is a Dirac fermion, there is potential to realize a theory with asymmetric dark matter. We leave it to future studies to explore these possibilities.

\subsection{Dark Matter}

Here we examine the DM matter candidate in this model. We first discuss the stability which results from an accidental global symmetry of the Lagrangian and identify the DM as a heavy mostly singlet neutrino. This global symmetry is a consequence of the particle content and underlying lepton gauge symmetry, much in the same way that lepton number is an accidental global symmetry in the SM. We then discuss the various annihilation channels and calculate the relic abundance of the DM candidate to establish the allowed masses. We also discuss various other phenomenological features. 


\subsubsection{DM Candidate and Stability}

We begin by examining the neutrino sector once $\Phi$ and $H$ obtain expectation values which gives,

$$
\begin{gathered}
\mathcal{L} \supset-\frac{c_{\ell} v_{\phi}}{\sqrt{2}}\left(1+\frac{\phi_{o}}{v_{\phi}}\right) \bar{\nu}_{R}^{\prime \prime} \nu_{L}^{\prime}-\frac{c_{\nu} v_{\phi}}{\sqrt{2}}\left(1+\frac{\phi_{o}}{v_{\phi}}\right) \bar{\nu}_{L}^{\prime \prime} \nu_{R}^{\prime} \\
-\frac{y_{\nu}^{\prime \prime} v_{h}}{\sqrt{2}}\left(1+\frac{h_{o}}{v_{h}}\right) \bar{\nu}_{R}^{\prime \prime} \nu_{L}^{\prime \prime}-\frac{y_{\nu}^{\prime} v_{h}}{\sqrt{2}}\left(1+\frac{h_{o}}{v_{h}}\right) \bar{\nu}_{L}^{\prime} \nu_{R}^{\prime}+h . c .
\end{gathered}
$$

leading to the mass matrix,

$$
\mathcal{M}_{\nu}=\frac{1}{\sqrt{2}}\left(\begin{array}{cc}
c_{\ell} v_{\phi} & y_{\nu}^{\prime} v_{h} \\
y_{\nu}^{\prime \prime} v_{h} & c_{\nu} v_{\phi}
\end{array}\right)
$$

which can be diagonalized using the singular value decomposition $\mathcal{M}_{\nu D}=U_{L}^{\dagger} \mathcal{M}_{\nu} U_{R}$, where $\mathcal{M}_{\nu D}$ is a diagonal mass matrix with positive mass eigenvalues $m_{\nu_{X}}$ and $m_{\nu_{4}}$.

While the Yukawa couplings to $\Phi$ and $H$ break the global U(1) symmetries associated with $L^{\prime}$ and $L^{\prime \prime}$ explicitly, there is a residual $Z_{2}$ symmetry under which all heavy leptons are odd and all SM leptons are even, which is preserved after spontaneous breaking of the lepton number and electroweak gauge symmetries. Assuming that the new charged leptons are heavier, this residual global symmetry guarantees the stability of the lighter of the two neutrino mass eigenstates, opening up the possibility for dark matter.

In the limit where $y_{\nu}^{\prime} v_{h}, y_{\nu}^{\prime \prime} v_{h} \ll c_{\ell, \nu} v_{\phi}$, the mass eigenvalues are approximately given by

$$
\begin{aligned}
& m_{\nu_{X}} \approx \frac{1}{\sqrt{2}} c_{\nu} v_{\phi} \\
& m_{\nu_{4}} \approx \frac{1}{\sqrt{2}} c_{\ell} v_{\phi}
\end{aligned}
$$

In this limit, the eigenstate $\nu_{4}$ is mostly composed of the electroweak doublet neutrinos $\nu_{R}^{\prime \prime}$ and $\nu_{L}^{\prime}$, while $\nu_{X}$ is a combination of the singlets $\nu_{L}^{\prime \prime}$ and $\nu_{R}^{\prime}$ and with tiny couplings to the SM $W^{ \pm}$ and $Z$ bosons. Since the doublet neutrino $\nu_{4}$ couples directly to the $Z$ boson, direct detection 
experiments render it unacceptable as a DM candidate. Therefore we require $c_{\nu}<c_{\ell}$, such that $\nu_{X}$ is the DM candidate. Of course $\nu_{4}$ must be able to decay which means that at least one of the Yukawa couplings $y_{\nu}^{\prime}, y_{\nu}^{\prime \prime}$ should be nonzero to allow $\nu_{4}$ to decay into a Higgs boson and $\nu_{X}$. Nonetheless, this requirement allows the $y_{\nu}$ 's to be small enough so as to be completely irrelevant in the discussion below.

\subsubsection{Annihilation Channels}

In [60], annihilation through the interactions generated by $y_{\nu}^{\prime}, y_{\nu}^{\prime \prime}$ was shown to give the correct relic abundance for DM with dominantly Majorana masses $\lesssim 100 \mathrm{GeV}$. Here, because direct detection constraints require $y_{\nu}^{\prime}, y_{\nu}^{\prime \prime}$ to be tiny, one would have to either rely on co-annihilation with one of the charged leptons or annihilation through a nearly on-shell Higgs. We instead will assume in the following that these couplings are too tiny to affect the DM phenomenology directly, although they do play a role in direct and indirect searches as well as LHC phenomenology, to be described below.

Compared to [60], there are additional annihilation channels for $\nu_{X}$ into SM leptons. In particular, since $\nu_{X}$ is a Dirac fermion, annihilation through a vector boson is $s$-wave and unsuppressed, in contrast to the case of Majorana DM. Indeed, the left- and right-handed components of $\nu_{X}$ carry lepton number $L^{\prime \prime}$ and $L^{\prime}$, respectively, and $L^{\prime}-L^{\prime \prime}=-3$ implies a non-vanishing coupling of $\nu_{X}$ to $Z_{L}$, allowing $\nu_{X} \bar{\nu}_{X}$ to annihilate into SM leptons through $s$ channel $Z_{L}$ exchange, shown in the top diagram of Fig. 4.3. There are additional annihilation channels which arise through mixing in the neutrino as well as in the Higgs sectors. We discuss the various annihilation modes in more detail below, assuming that $\nu_{X}$ is mostly singlet with at most a small doublet component, i.e. $y_{\nu}^{\prime} v_{h}, y_{\nu}^{\prime \prime} v_{h} \ll c_{\nu} v_{\phi}$.

If $\nu_{X}$ acquires a small doublet component through nonzero $y_{\nu}^{\prime}, y_{\nu}^{\prime \prime}$ couplings, annihilation into SM particles through $Z$ or $h$ exchange becomes possible, but again we will assume that these 


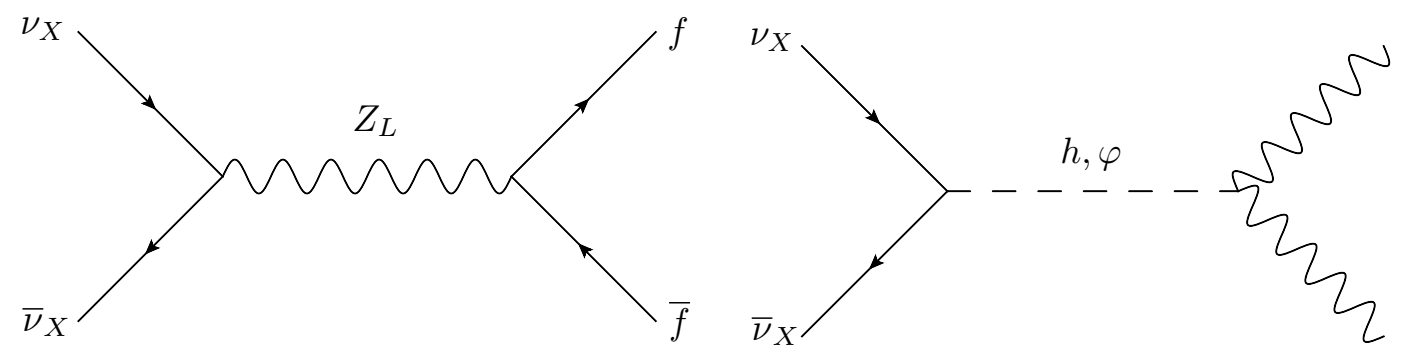

Figure 4.3. Diagrams leading to $s$-channel $\nu_{X} \bar{\nu}_{X}$ annihilation into SM states through exchange of $Z_{L}, h$, or $\phi$.

couplings are sufficiently small such that these annihilation channels can be neglected. This is also required since otherwise a large direct detection cross section through $Z$ boson exchange would be induced. At the same time this suppresses annihilation into $W^{+} W^{-}$through a heavy charged lepton exchanged in the $t$-channel.

The dark matter also couples to the singlet scalar $\phi_{o}$ with a strength $c_{\nu} \approx \sqrt{2} m_{\nu_{X}} / v_{\phi}$. When the Higgs mixing angle $\theta$ is nonzero this will allow annihilation into SM particles through $s$-channel exchange of $h$ and $\phi$, shown in the bottom diagram of Fig. 4.3. While not generally negligible, the contribution of these annihilation channels turns out to be suppressed compared to the $Z_{L}$ channel in the regime of interest where $v_{\phi} \sim 1.7 \mathrm{TeV}$ and $\mathrm{DM} m_{\nu_{X}} \sim v_{h}$, leading to somewhat small values for $c_{\nu}$. Furthermore the $Z_{L}$ channel leads to unsuppressed annihilation into all SM leptons, while most of the scalar channels are suppressed by the small Yukawa couplings of the SM quarks and leptons. We thus expect annihilation through $Z_{L}$ to be the dominant contribution to the relic abundance in this regime. Note also that in this regime we have $m_{\nu_{X}} \ll M_{Z_{L}}$ which as we will see leads to a relic abundance which is largely independent of the lepton gauge coupling $g^{\prime}$ (see Eq.4.22). 


\subsubsection{Relic Abundance}

Motivated by the requirement for small $y_{\nu}^{\prime}, y_{\nu}^{\prime \prime}$, we first consider the dominant annihilation through the $Z_{L}$ into SM lepton pairs, and then demonstrate that scalar exchange is unlikely to change the over-all picture. The relevant interactions come from Eq. (4.4) which before lepton number and electroweak symmetry breaking can be written as,

$$
\mathcal{L} \supset g^{\prime} Z_{L \mu}\left(L^{\prime \prime} \bar{\nu}_{R}^{\prime \prime} \gamma^{\mu} \nu_{R}^{\prime \prime}+L^{\prime} \bar{\nu}_{L}^{\prime} \gamma^{\mu} \nu_{L}^{\prime}+\bar{l} \gamma^{\mu} l\right)
$$

where $l$ runs over SM leptons all of which have $L=1$, which implies that the left and right handed couplings of the SM leptons to $Z_{L}$ are equal. This is in contrast to the case for the exotic leptons since $L^{\prime} \neq L^{\prime \prime}$. After lepton number breaking and rotating to the mass basis Eq. (4.17) becomes

$$
\mathcal{L} \supset g^{\prime} Z_{L \mu}\left(\bar{\nu}_{X} \gamma^{\mu}\left(L^{\prime \prime} P_{R}+L^{\prime} P_{L}\right) \nu_{X}+\bar{l} \gamma^{\mu} l\right)
$$

where $P_{R}$ and $P_{L}$ are the right and left projection operators respectively and we have neglected any mixing between $\nu_{X}$ and $\nu_{4}$ generated by $y_{\nu}^{\prime}, y_{\nu}^{\prime \prime}$. Using Eq. 4.18 a straight forward calculation of the diagram in Fig. 4.3 gives the annihilation cross section,

$$
\sigma=\frac{g^{\prime 4}\left(\left(L^{\prime 2}+L^{\prime \prime 2}\right)\left(s-m_{\nu_{X}}^{2}\right)+6 L^{\prime} L^{\prime \prime} m_{\nu_{X}}^{2}\right)}{8 \pi\left(1-4 m_{\nu_{X}}^{2} / s\right)^{1 / 2}\left(\left(M_{Z_{L}}^{2}-s\right)^{2}+M_{Z_{L}}^{2} \Gamma_{Z_{L}}^{2}\right)}
$$

where an overall factor of 6 is implicit for the three generations of charged leptons and neutrinos in the SM. As is well known, the annihilation cross section $\langle\sigma v\rangle$ is well approximated by a non-relativistic expansion, $s=4 m_{\nu_{X}}^{2}+m_{\nu_{X}}^{2} v^{2}$, and expanding the annihilation cross section in powers of $v$ to give $\langle\sigma v\rangle=a+b\left\langle v^{2}\right\rangle+\mathcal{O}\left(\left\langle v^{4}\right\rangle\right)$ [83]. Expanding Eq. 4.19] we obtain

$$
a=\frac{3 g^{\prime 4} R^{4}\left(L^{\prime}+L^{\prime \prime}\right)^{2}}{4 \pi m_{\nu_{X}}^{2}\left(1-4 R^{2}\right)^{2}}
$$


for the velocity independent coefficient. Note, this is in contrast to the case of Majorana dark matter annihilating through a gauge boson, in which case $a=0$ up to corrections that are suppressed by the final state fermion masses. For the $\left\langle v^{2}\right\rangle$ coefficient we have

$$
b=\frac{g^{4} R^{4}\left(\left(L^{\prime 2}+L^{\prime \prime 2}\right)\left(11+4 R^{2}\right)+L^{\prime} L^{\prime \prime}\left(6+72 R^{2}\right)\right)}{32 \pi m_{\nu_{X}}^{2}\left(1-4 R^{2}\right)^{3}}
$$

Here we have defined $R=m_{\nu_{X}} / M_{Z_{L}}$ and neglected terms of order $\Gamma_{Z_{L}} / M_{Z_{L}}$. In general the contribution from $a$ will dominate since the contribution from $b$ is suppressed by the relatively small value of $v^{2}$ at freeze-out. It is useful to consider the limit of heavy $Z_{L}$ mass compared to the DM mass, or $R \ll 1$. Keeping only the leading term after expanding in powers of $R$ we have

$$
a \approx \frac{3 g^{\prime 4}\left(L^{\prime}+L^{\prime \prime}\right)^{2} R^{4}}{4 \pi m_{\nu_{X}}^{2}}+\mathcal{O}\left(R^{6}\right)
$$

Since $M_{Z_{L}}=3 g^{\prime} v_{\phi}$, the dependence on the gauge coupling $g^{\prime}$ cancels in the leading term, as is usual for the contact interaction that describes vector exchange at low energies. For a fixed choice of the quantum numbers $L^{\prime}$ and $L^{\prime \prime}$, the annihilation rate is therefore largely determined by the ratio $m_{\nu_{X}}^{2} / v_{\phi}^{4}$.

From these results a good approximation for the relic density can be obtained e.g. using the procedure presented in [83]. We have opted instead to implement the model into the numerical code MICROMEGAS [85]. Not only does this facilitate the exploration of regions of parameter space where the $\mathcal{O}\left(v^{2}\right)$ expansion breaks down, but it also simplifies the computation of direct and indirect detection signals. The approximate calculation of the relic density following [83] was used as validation of the MICROMEGAS implementation of the model. The resulting relic density (including all sub-leading effects) is shown as a function of $m_{\nu_{X}}$ and $v_{\phi}$, for a few choices of $L^{\prime}$, in Figure 4.4. The LEP II constraints on $v_{\phi}$ require dark matter masses greater than about 


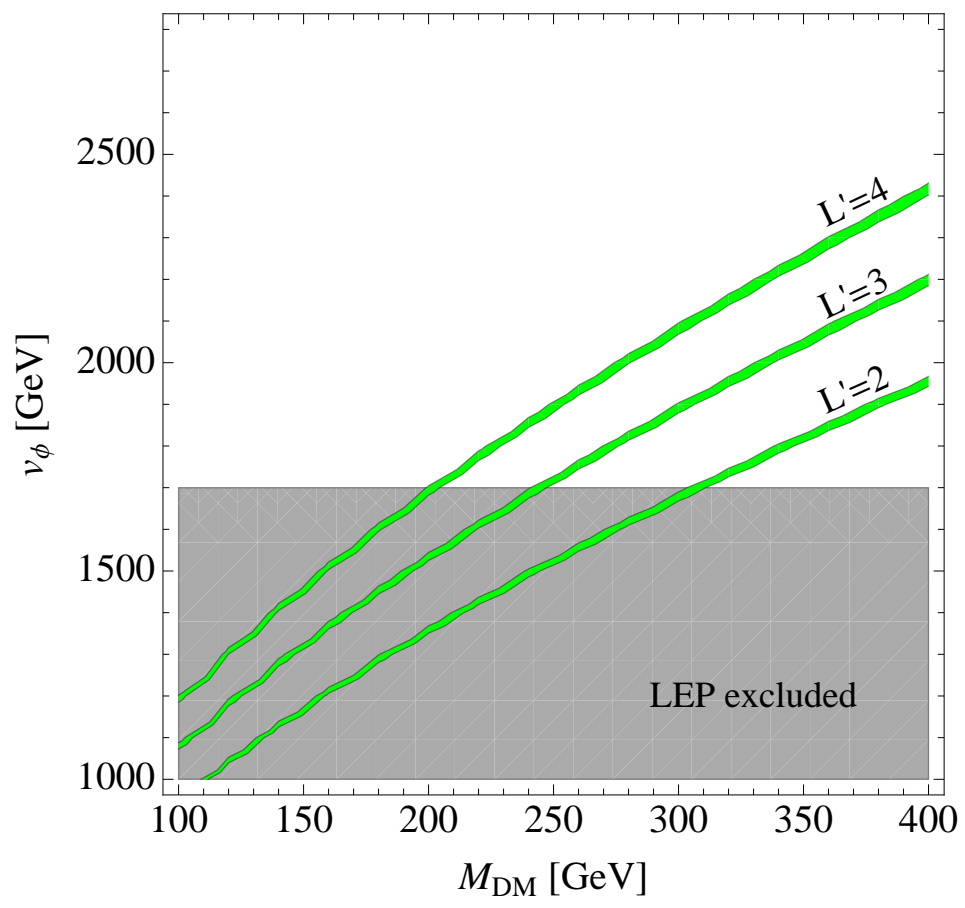

Figure 4.4. Relic density as a function of DM mass and vev $v_{\phi}$, in the absence of mixing and taking $M_{D M}=m_{\nu_{X}}$. The green bands indicate regions in agreement with the measured value of $\Omega h^{2}=0.120 \pm 0.003$ [84] for different choices of $L^{\prime}$, as indicated in the figure.

$200 \mathrm{GeV}$, and (depending on $L^{\prime}$ ), a thermal relic density enforces a tight correlation between $v_{\phi}$ and $m_{\nu_{X}}$.

In the limit $y_{\nu}^{\prime}, y_{\nu}^{\prime \prime} \approx 0, \mathrm{DM}$ couples to $h$ and $\phi$ through $c_{\nu}$ and the Higgs mixing,

$$
\mathcal{L} \supset \frac{c_{\nu}}{\sqrt{2}}\left(c_{\theta} \phi-s_{\theta} h\right) \bar{\nu}_{X} \nu_{X}
$$

where we have used Eq. (4.8). These couplings allow the DM to annihilate through the bottom diagram shown in Fig. 4.3. Since dark matter masses of order the weak scale require a relatively small $c_{\nu}$, annihilation through Higgs exchange only has a small effect on the relic density. On the other hand it is crucial for direct detection which will be discussed in the next section. 

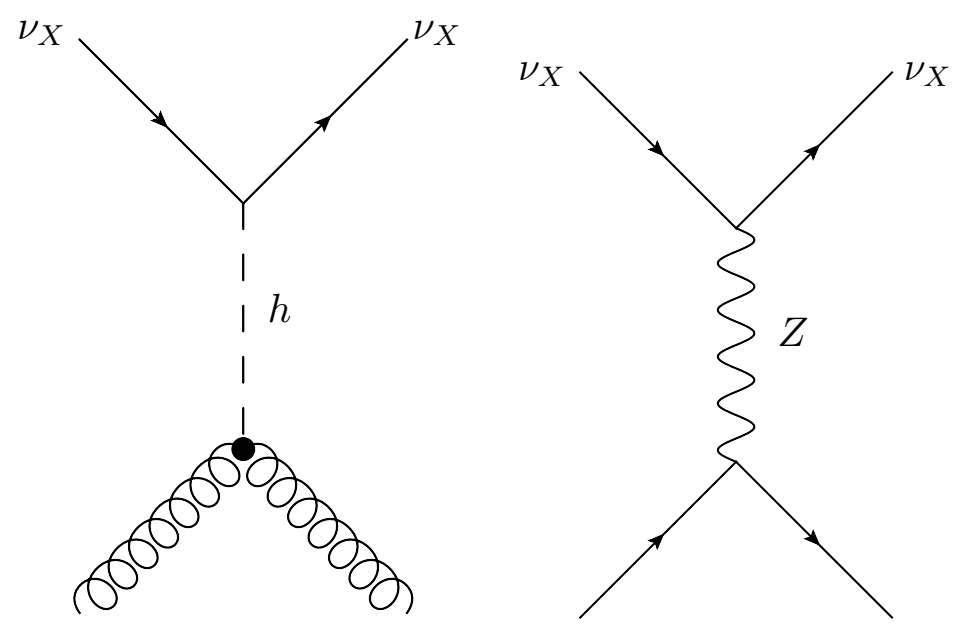

Figure 4.5. Diagrams leading to scattering with nucleons mediated by exchange of a Higgs or $Z$ boson.

\subsubsection{Direct and Indirect Detection}

In the limit $y_{\nu}^{\prime}, y_{\nu}^{\prime \prime} \rightarrow 0$ and negligible mixing in the Higgs sector, the dark matter couples to SM leptons through $Z_{L}$, but has no tree level interactions with quarks. This is a challenging situation for dark matter direct detection experiments, because of the wave function suppression to scatter off of atomic electrons or loop suppression of the induced dark matter dipole moment [86]. Consequently, even a small amount of $Z-Z_{L}$ or $H-\Phi$ mixing can dominate the rate, which effectively disconnects the expectations at direct detection experiments from the relic density.

Higgs exchange leads to spin-independent scattering with nuclei. We compute the rate as a function of the DM mass and Higgs mixing angle $\sin \theta$ using MICROMEGAS and present the results in Figure 4.6 for DM masses 100-400 GeV. For moderate Higgs mixing, The DM-nucleon cross section lies about one order of magnitude below the current best limit from the XENON100 experiment, but is well in reach of second generation DM direct detection experiments such as LZ [87]. 


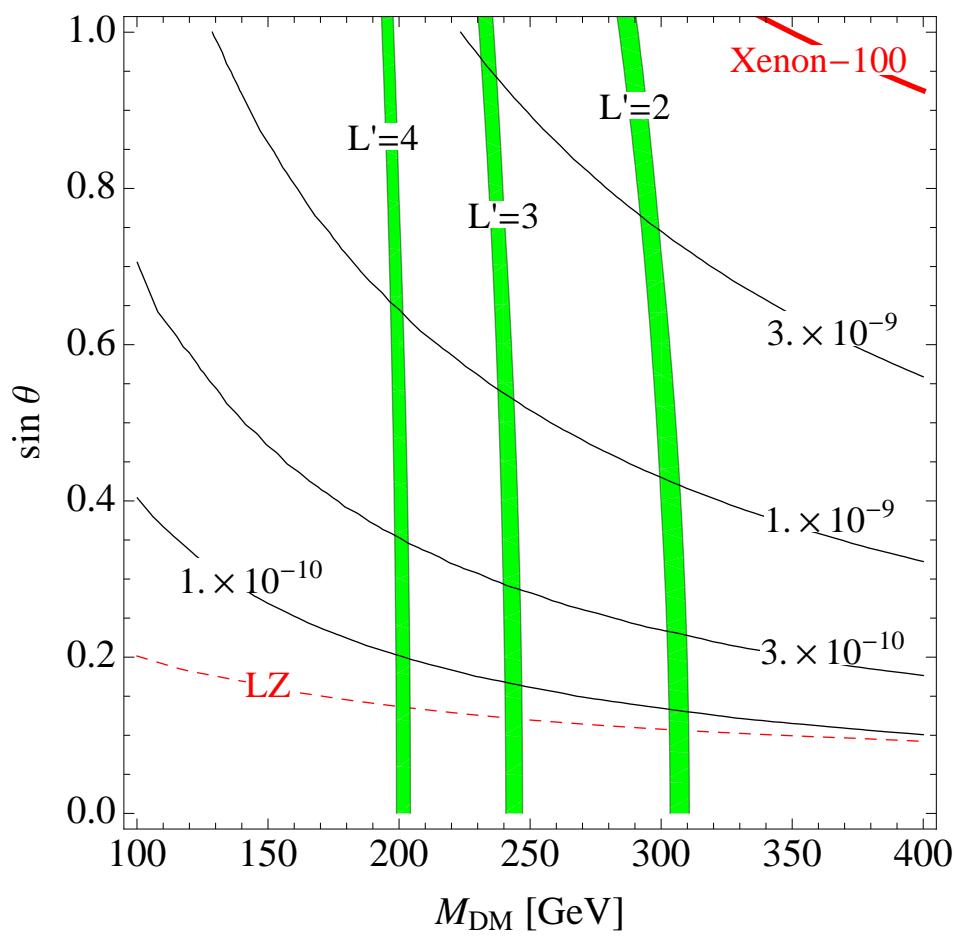

Figure 4.6. DM-nucleon cross section in $\mathrm{pb}$, as a function of the Higgs mixing angle $\sin \theta$ and of the DM mass, for $v_{\phi}=1.7 \mathrm{TeV}$ and $M_{D M}=$ $m_{\nu_{X}}$. The solid red line indicates the current limit from the Xenon-100 experiment [76], while the dashed red line indicates the projected reach of the LZ experiment [87. The green bands indicate regions with correct relic density for different values of $L^{\prime}$.

Z-boson exchange induces a large DM-neutron cross section due to the sizable coupling of the $Z$ to light quarks. We parameterize the coupling of the $Z$-boson to the DM as,

$$
\mathcal{L} \supset \epsilon^{\prime} g^{\prime} Z_{\mu} \bar{\nu}_{X} \gamma^{\mu}\left(L^{\prime \prime} P_{R}+L^{\prime} P_{L}\right) \nu_{X},
$$

where $\epsilon^{\prime}$ is either induced by $Z-Z^{\prime}$ mixing or by nonzero neutrino Yukawa couplings $y_{\nu}^{\prime}, y_{\nu}^{\prime \prime}$. The upper bound on $\epsilon^{\prime}$ from direct detection for $L^{\prime}=2$ is shown in Fig. 4.7, for DM masses $100-400 \mathrm{GeV}$. One can see that for $g^{\prime}=0.5$ and $v_{\phi}=1.7 \mathrm{TeV}$, direct detection requires roughly $\epsilon^{\prime} \lesssim 1-2 \times 10^{-4}$ depending on the DM mass. In the limit $y_{\nu}^{\prime}, y_{\nu}^{\prime \prime} \approx 0, \epsilon^{\prime}$ is due solely to $Z-Z_{L}$ 


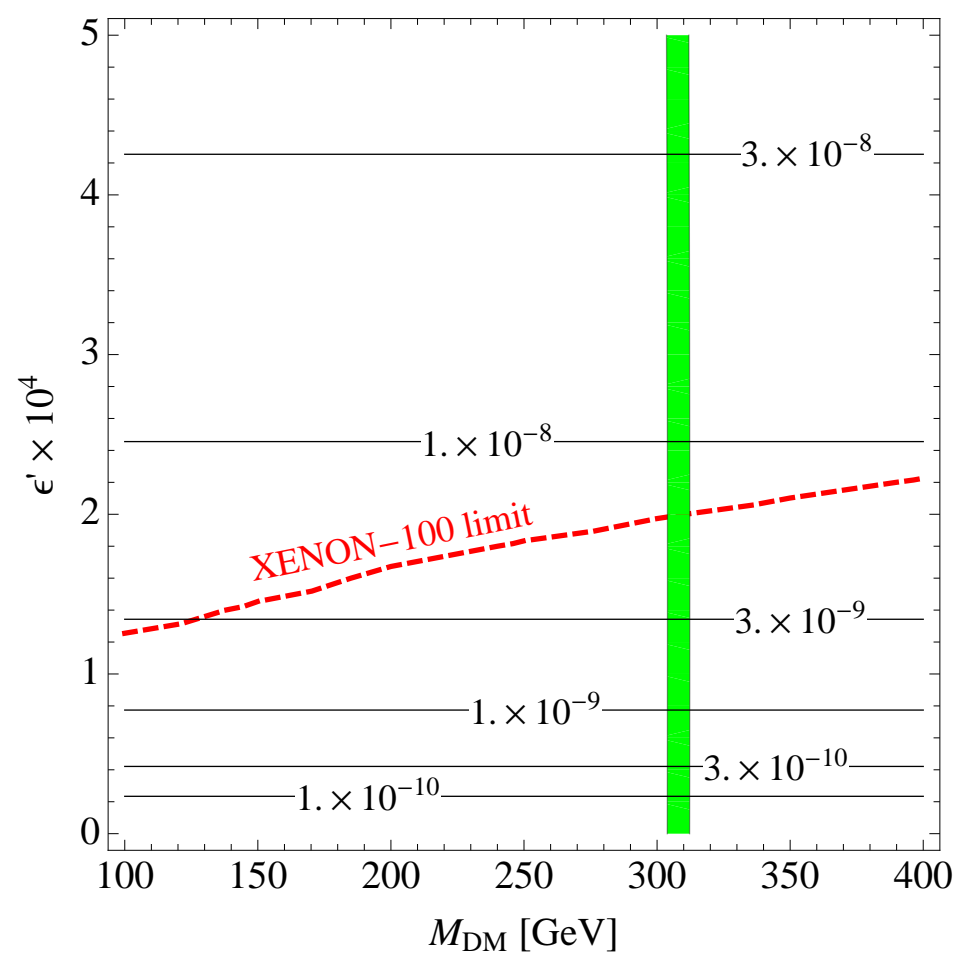

Figure 4.7. DM-nucleon cross section in pb, as a function of the DM-Z coupling parameter $\epsilon^{\prime}$ and of the DM mass (where $M_{D M}=m_{\nu_{X}}$ ), for $v_{\phi}=$ $1.7 \mathrm{TeV}, L^{\prime}=2$ and $g^{\prime}=0.5$ which implies $M_{Z_{L}}=2.55 \mathrm{TeV}$. The red dashed line indicates the current limit from the XENON-100 experiment.

mixing and gives $\epsilon^{\prime}=\sin \xi$ as defined in Eq.(4.5). Since $M_{Z_{L}}=3 g^{\prime} v_{\phi}=2.55 \mathrm{TeV}$, Eq. (4.5) and Fig 4.2 together imply that direct detection signals roughly 20 times below the current bound can be obtained for a gauge kinetic mixing parameter (see Eq.4.4) of $\epsilon \sim 7 \times 10^{-3}$, within range of future direct detection experiments [87].

Dark matter can also be observed indirectly, by searching for the products of DM annihilation. Here, the dark matter annihilates predominantly into charged leptons or neutrinos. While there is a large rate into positrons, it is characterized by roughly the thermal relic cross section and is thus quite a bit too small to account for the anomalous positron fraction observed by PAMELA [88, Fermi [89], and AMS-02 [90]. At the same time, contributions to the anti-proton flux are very tiny, evading constraints from PAMELA [91]. 
Annihilation into charged leptons will also produce gamma rays as secondaries. Currently, the tightest constraints on such production are from the Fermi LAT null observations of dwarf spheroidal galaxies [92], which are just short of being able to rule out thermal cross sections for dark matter masses around a few 10's of GeV based on one sixth of the annihilations producing $\tau^{+} \tau^{-}$. In the near future, such constraints are only relevant for $\nu_{X}$ dark matter which has been produced non-thermally.

Dark matter may also annihilate directly into $\gamma \gamma$ and/or $\gamma Z$ at loop level, providing monochromatic gamma ray lines, whose distinctive energy profile can help compensate for a tiny rate. Predictions for the class of models including $U(1)_{L}$ were studied in 93], where it was found that $\gamma \gamma, \gamma Z$, and $\gamma \phi$ (if kinematically accessible) final states can be generated. The largest signal is likely to be $\gamma \phi$, which is expected to be at least an order of magnitude below the current Fermi bounds [94, but may be visible to future experiments.

The rate for dark matter to be captured in the Sun or Earth and then annihilate into high energy neutrinos is controlled by the spin-dependent cross section which in turn is controlled by the degree of $Z-Z_{L}$ mixing. Thus, despite a large annihilation fraction into SM neutrinos, the precision constraints render it difficult to imagine an observable rate at ICECUBE in the near future 95 .

\subsection{LHC Phenomenology and Constraints}

The presence of new particles required by the $U(1)_{L}$ gauge symmetry leads to a variety of potentially interesting LHC phenomenology. In this section we discuss various aspects of the phenomenology of this model as well as the relevant constraints coming from the LHC. We also examine in more detail the charged lepton sector and its effects on the Higgs decays. 


\subsubsection{Exotic Charged Lepton Sector}

Once $\Phi$ and $H$ obtain expectation values, the Lagrangian for the exotic charged lepton sector becomes,

$$
\begin{gathered}
\mathcal{L} \supset-\frac{c_{\ell} v_{\phi}}{\sqrt{2}}\left(1+\frac{\phi_{o}}{v_{\phi}}\right) \bar{e}_{R}^{\prime \prime} e_{L}^{\prime}-\frac{c_{e} v_{\phi}}{\sqrt{2}}\left(1+\frac{\phi_{o}}{v_{\phi}}\right) \bar{e}_{L}^{\prime \prime} e_{R}^{\prime} \\
-\frac{y_{e}^{\prime \prime} v_{h}}{\sqrt{2}}\left(1+\frac{h_{o}}{v_{h}}\right) \bar{e}_{R}^{\prime \prime} e_{L}^{\prime \prime}-\frac{y_{e}^{\prime} v_{h}}{\sqrt{2}}\left(1+\frac{h_{o}}{v_{h}}\right) \bar{e}_{L}^{\prime} e_{R}^{\prime}+\text { h.c. }
\end{gathered}
$$

which gives a mass matrix of the same form as that found in the neutrino sector,

$$
\mathcal{M}_{e}=\frac{1}{\sqrt{2}}\left(\begin{array}{cc}
c_{\ell} v_{\phi} & y_{e}^{\prime \prime} v_{h} \\
y_{e}^{\prime} v_{h} & c_{e} v_{\phi}
\end{array}\right)
$$

Again we can diagonalize via $\mathcal{M}_{e D}=U_{L}^{\dagger} \mathcal{M}_{e} U_{R}$ to obtain the mass eigenvalues and eigenstates. The Lagrangian in Eq. 4.25 also leads to the interaction matrices for $\phi_{o}$ and $h_{o}$ given by,

$$
\mathcal{N}_{e}^{h}=\frac{v_{h}}{\sqrt{2}}\left(\begin{array}{cc}
0 & y_{e}^{\prime \prime} \\
y_{e}^{\prime} & 0
\end{array}\right), \mathcal{N}_{e}^{\phi}=\frac{v_{\phi}}{\sqrt{2}}\left(\begin{array}{cc}
c_{\ell} & 0 \\
0 & c_{e}
\end{array}\right),
$$

which upon the rotation performed to diagonalize $\mathcal{M}_{e}$ gives interaction matrices in the mass basis defined as $\mathcal{V}_{\phi}=U_{L}^{\dagger} \mathcal{N}_{e}^{\phi} U_{R}$ and $\mathcal{V}_{h}=U_{L}^{\dagger} \mathcal{N}_{e}^{h} U_{R}$. These matrices dictate the couplings of the exotic leptons to $\phi$ and $h$. We note also that Eq.4.26 is the same mass matrix in the charged lepton sector considered in [60], with the difference being that in this model there are no explicit mass terms. In particular, when $v_{h}, v_{\phi} \rightarrow 0$ all masses go to zero, which makes the gauged lepton number model more constrained and relates the electroweak and lepton breaking scales to the rate of Higgs decay to di-photons, as we will see below. 
A useful simplifying limit is $c_{\ell} \approx c_{e} \equiv c_{e}$ and $y_{e}^{\prime} \approx y_{e}^{\prime \prime} \equiv y_{e}$ in which case the charged leptons are maximally mixed and one obtains the simple relations for the mass eigenvalues,

$$
\begin{aligned}
& m_{e_{1}} \approx \frac{1}{\sqrt{2}}\left(c_{e} v_{\phi}-y_{e} v_{h}\right) \\
& m_{e_{2}} \approx \frac{1}{\sqrt{2}}\left(c_{e} v_{\phi}+y_{e} v_{h}\right),
\end{aligned}
$$

where we have assumed $c_{e} v_{\phi}>y_{e} v_{h}$. Thus we see that for fixed $y_{e}$ and $v_{\phi}$, the mass of the charged leptons is controlled by $c_{e}$. Along with the scalar mixing discussed in Sec. 4.2 .2 we now have the pieces necessary for examining the modification to Higgs decays.

\subsubsection{Modifications of Higgs Decays}

Assuming that the Higgs can not decay directly into new particles, the primary effect of the new lepton sector on Higgs decays will be through loop effects. From the discussion on Higgs mixing in Sec 4.2 .2 , we can write the modification of the SM Higgs partial width as,

$$
\begin{aligned}
& \epsilon_{i} \equiv \frac{\Gamma_{h i}}{\Gamma_{h_{o} i}^{S M}}=\frac{|\mathcal{M}(h \rightarrow i)|^{2}}{\left|\mathcal{M}\left(h_{o} \underset{S M}{\longrightarrow} i\right)\right|^{2}} \\
& =\frac{c_{\theta}^{2}\left|\mathcal{M}\left(h_{o} \rightarrow i\right)-t_{\theta} \mathcal{M}\left(\phi_{o} \rightarrow i\right)\right|^{2}}{\left|\mathcal{M}\left(h_{o} \underset{S M}{\longrightarrow} i\right)\right|^{2}},
\end{aligned}
$$

where we have used Eq.4.8 and $\Gamma_{h_{o} i}^{S M}$ is the SM partial width to a final state $i$ and $\Gamma_{h i}$ is the partial width for $h$ to decay into $i$. The rate expected at the LHC relative to the SM can be written as,

$$
\mu_{i}=\frac{\sigma(j \rightarrow h)}{\sigma\left(j \underset{S M}{\longrightarrow} h_{o}\right)} \frac{\mathcal{B}(h \rightarrow i)}{\mathcal{B}\left(h_{o} \underset{S M}{\longrightarrow}\right)}=\epsilon_{j} \frac{\Gamma_{h_{o}}^{S M}}{\Gamma_{h}} \epsilon_{i},
$$

where we have made use of the narrow width approximation, $\mathcal{B}$ signifies the branching fraction, and the production channels are labeled $j=V V, g g$. We also define $\Gamma_{h_{o}}^{S M}$ as the total SM Higgs 
width and $\Gamma_{h}$ as the total decay width for the mass eigenstate $h$. Since this model does not contain any new colored particles the only new effects entering $\epsilon_{g g}$ are through Higgs mixing which gives $\epsilon_{g g} \approx c_{\theta}^{2}$. Since $Z Z$ and $W W$ already occur at tree level in the SM, we assume the loop corrections due to the new leptons are negligible which implies the only effect again comes from Higgs mixing, which gives $\epsilon_{Z Z}=\epsilon_{W W} \sim c_{\theta}^{2}$. Similarly for the SM Higgs Yukawa interactions we have $\epsilon_{Y} \sim c_{\theta}^{2}$.

This leaves the $Z \gamma$ and $\gamma \gamma$ channels, which first occur at one loop in the SM, as the most promising possibilities for these effects to manifest themselves. However, in Refs. [60, 96] the modification to $Z \gamma$ was shown to be only $\sim 5 \%$ for a corresponding $\gamma \gamma$ enhancement of $\sim 50 \%$, and to good approximation $\epsilon_{Z \gamma} \sim c_{\theta}^{2}$. Thus, in addition to the universal $c_{\theta}^{2}$ suppression from Higgs mixing, the only additional modifications to the total decay width comes from the $\gamma \gamma$ channel through loops of exotic charged leptons. Since for the modifications we are interested in $\Gamma_{h \gamma \gamma} \ll \Gamma_{h}$ this implies $\Gamma_{h_{o}}^{S M} / \Gamma_{h} \approx c_{\theta}^{-2}$ which will cancel with the $c_{\theta}^{2}$ in the production channels $\epsilon_{g g, V V}$. This gives finally for the relative rates $\mu_{i}=c_{\theta}^{2}$ for $i \neq \gamma \gamma$ and for the final modified diphoton signal strength,

$$
\mu_{\gamma \gamma}=\epsilon_{\gamma \gamma}
$$

Using the approach and conventions of [97], which examined the similar $g g \rightarrow h$ process, we can go on to obtain the exotic charged lepton contributions to the $h \rightarrow \gamma \gamma$ amplitudes (omitting photon polarization vectors),

$$
\begin{aligned}
& \mathcal{M}^{\mu \nu}\left(h_{o} \rightarrow \gamma \gamma\right)=\left(\frac{\alpha}{2 \pi v_{h}}\right) \sum_{i} \frac{\left(\mathcal{V}_{h}\right)_{i i} F_{F}\left(\tau_{e_{i}}\right)}{m_{e_{i}}}\left(p_{1}^{\nu} p_{2}^{\mu}-\frac{m_{h}^{2}}{2} g^{\mu \nu}\right) \\
& \mathcal{M}^{\mu \nu}\left(\phi_{o} \rightarrow \gamma \gamma\right)=\left(\frac{\alpha}{2 \pi v_{\phi}}\right) \sum_{i} \frac{\left(\mathcal{V}_{\phi}\right)_{i i} F_{F}\left(\tau_{e_{i}}\right)}{m_{e_{i}}}\left(p_{1}^{\nu} p_{2}^{\mu}-\frac{m_{h}^{2}}{2} g^{\mu \nu}\right),
\end{aligned}
$$


where the index $i=1,2$ runs over the exotic charged lepton mass eigenstates found after diagonalizing the mass matrix in Eq. 4.26), and $F_{F}$ are the fermonic loop functions with $\tau_{e_{i}}=$ $m_{h}^{2} / 4 m_{e_{i}}^{2}$ as defined in [97]. Note that the amplitudes in Eq. 4.32 are evaluated at $m_{h_{o}}=m_{h}$ and $m_{\phi_{o}}=m_{h}$ where $m_{h}$ is the physical scalar mass.

Using Eq.4.29)-4.32 we obtain,

$$
\begin{aligned}
& \mu_{\gamma \gamma}=\frac{\left|\frac{c_{\theta}}{v_{h}}\left(F_{S M}+\sum_{i} \frac{\left(\mathcal{V}_{h}\right)_{i i}}{m_{e_{i}}} F_{F}\left(\tau_{e_{i}}\right)\right)-\frac{s_{\theta}}{v_{\phi}}\left(\sum_{i} \frac{\left(\mathcal{V}_{\phi}\right)_{i i}}{m_{e_{i}}} F_{F}\left(\tau_{e_{i}}\right)\right)\right|^{2}}{\left|F_{S M} / v_{h}\right|^{2}} \\
& =c_{\theta}^{2}\left|\left(1+F_{S M}^{-1} \sum_{i} \frac{\left(\mathcal{V}_{h}\right)_{i i}}{m_{e_{i}}} F_{F}\left(\tau_{e_{i}}\right)\right)-t_{\theta}\left(F_{S M}^{-1} \frac{v_{h}}{v_{\phi}} \sum_{i} \frac{\left(\mathcal{V}_{\phi}\right)_{i i}}{m_{e_{i}}} F_{F}\left(\tau_{e_{i}}\right)\right)\right|^{2},
\end{aligned}
$$

where $F_{S M}$ is the SM loop function which includes the dominant and negative $W^{ \pm}$boson contribution as well as the smaller and positive $t$-quark, which sum to give numerical value of $\sim-6.5$ for $m_{h}=125 \mathrm{GeV}$. Note only the diagonal entries in the interaction matrices $\left(\mathcal{V}_{h}\right)_{i i}$ and $\left(\mathcal{V}_{\phi}\right)_{i i}$ contribute in the $h \rightarrow \gamma \gamma$ loop.

After the approximations leading to the masses in Eq.44.28, which give $\left(\mathcal{V}_{\phi}\right)_{11}=\left(\mathcal{V}_{\phi}\right)_{22} \approx$ $c_{e} v_{\phi} / \sqrt{2}$ and $\left(\mathcal{V}_{h}\right)_{11}=-\left(\mathcal{V}_{h}\right)_{22} \approx-y_{e} v_{h} / \sqrt{2}$, we obtain (approximately) for the modified signal strength,

$$
\mu_{\gamma \gamma} \simeq c_{\theta}^{2}\left|1-\frac{v_{h}}{\sqrt{2} F_{S M}}\left[y_{e}\left(\frac{F_{F}\left(\tau_{e_{1}}\right)}{m_{e_{1}}}-\frac{F_{F}\left(\tau_{e_{2}}\right)}{m_{e_{2}}}\right)+c_{e} t_{\theta}\left(\frac{F_{F}\left(\tau_{e_{1}}\right)}{m_{e_{1}}}+\frac{F_{F}\left(\tau_{e_{2}}\right)}{m_{e_{2}}}\right)\right]\right|^{2}
$$

where $m_{e_{1}, e_{2}}$ are given in Eq.4.28 and satisfy $m_{e_{1}}<m_{e_{2}}$. Remembering that $F_{S M}<0$ we see in the limit $t_{\theta} \rightarrow 0$ we have an enhancement in the diphoton rate in the presence of mostly vector-like leptons entering through the $h_{o}$ component of $h$. This is, of course, expected from the low energy Higgs theorems (see e.g. 60]). We see also that the contribution from Higgs mixing is constructive for $t_{\theta}>0$ and destructive for $t_{\theta}<0$ which also corresponds to the sign 
of the coupling $\lambda_{h p}$ in Eq.44.6). In the limit $y_{e} \rightarrow 0$ the enhancement enters entirely through Higgs mixing and thus requires large mixing angles and Yukawa coupling $c_{e}$. In the realistic limit $v_{\phi} \gg v_{h}$, the $e_{1}$ and $e_{2}$ become almost purely vector-like and again the contribution only enters through Higgs mixing via the $\phi_{o}$ component of $h$. However as $v_{\phi} \rightarrow \infty$ one also has $t_{\theta} \rightarrow 0$ and the $\phi_{o}$ contribution eventually decouples from the $h \rightarrow \gamma \gamma$ amplitude as $v_{\phi}$ is taken large. Eq. 4.34 is in agreement with [62] for the case where their explicit mass term is put to zero.

To avoid the constraints discussed in Sec. 4.2.5 we choose $v_{\phi}=1.7 \mathrm{TeV}$ and take the lightest charged lepton to have mass greater than $m_{\text {min }} \sim 100 \mathrm{GeV}$. Measurements of the Higgs decays at the LHC indicate rates consistent with the SM with the possibility of a slight, though not significant, enhancement in the diphoton channel [98]. Regardless this implies that these fermions must be mostly 'vector-like' since otherwise their effects would lead to destructive interference [60] with the SM contribution giving a reduced rate, which is disfavored. This allows us to write,

$$
m_{e_{1}}=\frac{c_{e} v_{\phi}-y_{e} v_{h}}{\sqrt{2}} \gtrsim m_{m i n}
$$

which leads to a condition on the Yukawa coupling,

$$
\frac{\sqrt{2} m_{\min }+y_{e} v_{h}}{v_{\phi}} \lesssim c_{e} \lesssim 4 \pi .
$$

where we have also indicated $4 \pi$ as the perturbative upper bound.

Since the mixing angle will affect all decay channels, we perform a fit to the full Higgs data [99, 100] set in the $c_{e}-\theta$ plane for fixed $y_{e}=0.8$ and $v_{\phi}=1.7 \mathrm{TeV}$. We show in Fig. 4.8 the $1,2,3 \sigma$ regions (purple) for the favored parameter space where the grey band shows the excluded region by LEP II for which $m_{e_{1}}<100 \mathrm{GeV}$. Values as large as $\theta \sim \pm 0.5$ give a good fit to the Higgs data, while larger values are disfavored due to the $\cos \theta$ suppression of the signal 
rates. We also show contours of the relative diphoton rate shown in the green curves, though it is also worth noting that with the current data, the diphoton rate has no significant impact on the quality of the fit. Negative values of the mixing angle correspond to $\lambda_{h p}<0$, which can potentially lead to vacuum instabilities. On the other hand, positive values of $\theta \sim 0.5$ where $\lambda_{h p}>0$ lead to no instability and as shown in [62] can be made consistent with constraints coming from the $S$ and $T$ parameters.

Choosing instead to fix $c_{e}=0.3$ and trading in $y_{e}$ for the lightest charged lepton mass, we can examine contours of $\mu_{\gamma \gamma}$ as a function of $m_{e_{1}}$ and $\theta$ as seen in Fig. 4.9. Since the DM mass serves as a lower bound on the charged lepton mass we see for the DM masses $\gtrsim 200 \mathrm{GeV}$ found in Sec 4.3 that modifications up to $\sim 10-20 \%$ can be obtained for $\theta \sim 0.3-0.4$ and $m_{e_{1}} \gtrsim 200 \mathrm{GeV}$. Of course one can lower this bound by considering larger values of $L^{\prime}$ as can be seen in Fig. 4.4, or by tuning the $Z_{L}$ mass such that the DM annihilation is resonantly enhanced.

Allowing $c_{e}$ and $y_{e}$ to vary instead while fixing $\theta=0.4$ and $v_{\phi}=1.7 \mathrm{TeV}$, we show $\mu_{\gamma \gamma}$ contours in the $c_{e}-y_{e}$ plane in Fig. 4.10. As can be seen, observable modifications can be obtained for $\mathcal{O}(1)$ values of the Yukawa couplings for which vacuum stability issues can be avoided [62]. For these ranges of Yukawa couplings, $m_{e_{1}}$ lies in the range $100-500 \mathrm{GeV}$, such that the exotic leptons can be produced at the LHC. We will discuss possible collider signatures below.

If one is willing to push the Yukawa couplings as large as the perturbative limit $\sim 4 \pi$, one can realize large deviations in $\mu_{\gamma \gamma}$ even for multi-TeV masses. In Figure 4.11, we show the deviation in the plane of $m_{e_{1}}-m_{e_{2}}$ for fixed $v_{\phi}=1.7 \mathrm{TeV}$, right above the LEP II limit. Even for a lightest exotic charged lepton with mass $m_{e_{1}} \sim 2-3 \mathrm{TeV}$, one can obtain appreciable modifications to the Higgs diphoton rate, reflecting the fact that the fermion masses here are purely the result of Yukawa couplings, and thus do not exhibit decoupling [102]. Of course, all exotic contributions to the $h \rightarrow \gamma \gamma$ amplitude decouple in the limit of $v_{\phi} \rightarrow \infty$. It should 


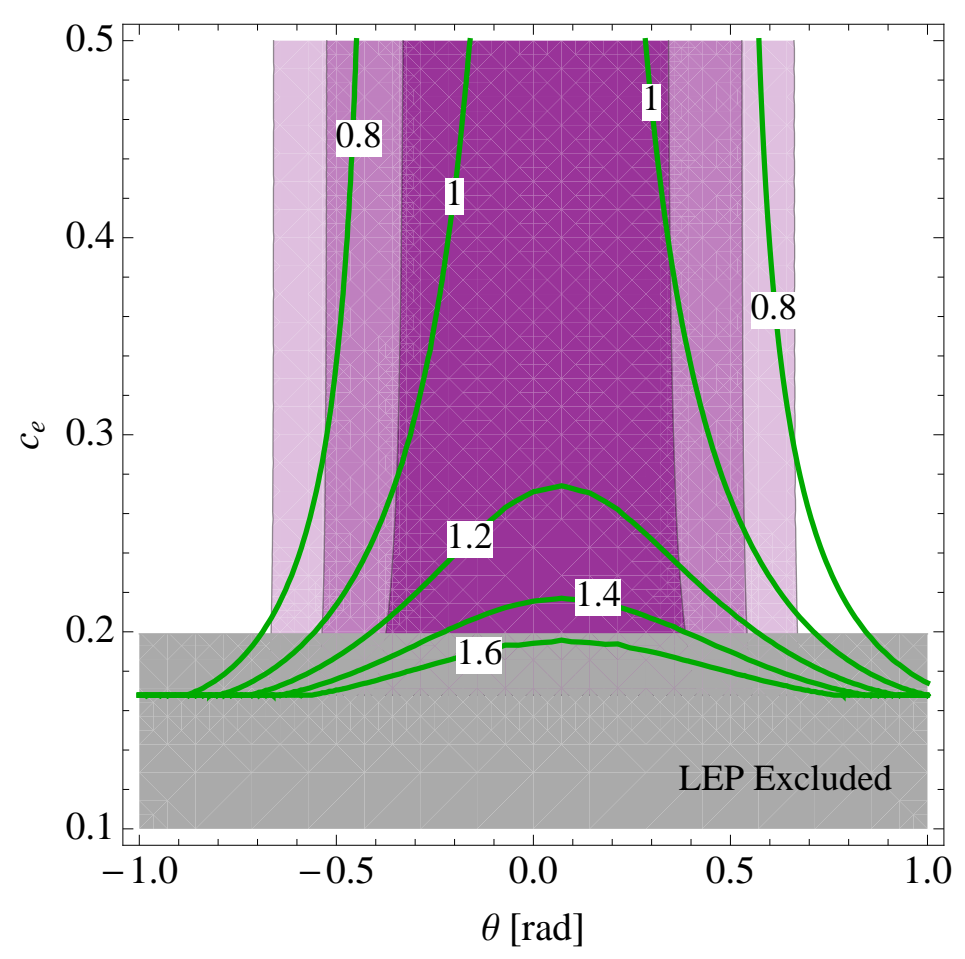

Figure 4.8. Fits to the full Higgs data set in the $c_{e}-\theta$ plane for $y_{e}=0.8$ and $v_{\phi}=1.7 \mathrm{TeV}$. Here the purple contours show the $1,2,3 \sigma$ regions while the grey band shows the LEP excluded region the green lines are contours of constant $\mu_{\gamma \gamma}$. Details on the fitting procedure can be found in [101].

also be noted that the required large Yukawa couplings can induce vacuum instabilities in the Higgs potential at scales close to the masses of the exotic leptons. Additional structures like supersymmetry would be required to restore vacuum stability. Some work in this direction recently appeared in $65,66,103$.

\subsubsection{Other Potential LHC Signatures}

Since the LHC is a hadron machine, weakly coupled extensions of the SM such as the model presented here are not heavily constrained by the current LHC data. Currently, constraints on the masses of the new leptons and of $Z_{L}$ mostly derive from the LEP experiments. Exotic charged leptons must be heavier than about $100 \mathrm{GeV}$ for consistency with direct search limits. The $Z_{L}$ 


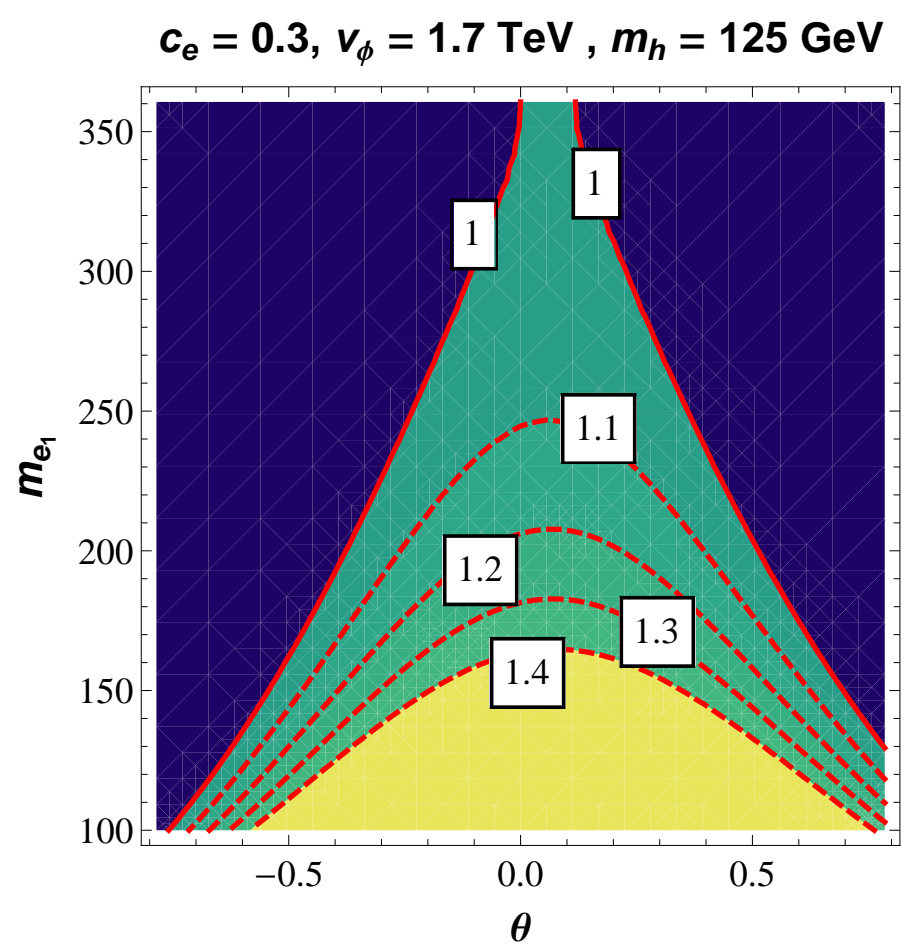

Figure 4.9. Contours of relative diphoton rate as a function of $\theta$ and lightest exotic charged lepton mass $m_{e_{1}}$.

mass should be larger than the LEP-2 center-of-mass energy of $209 \mathrm{GeV}$, and furthermore its coupling s subject to the constraint $M_{Z_{L}}=3 g^{\prime} v_{\phi}$ where $v_{\phi} \geq 1.7 \mathrm{TeV}$ (and we have neglected any kinetic mixing with the $Z$ boson).

One of the defining features of our model is $Z_{L}$, the gauge boson of the lepton number symmetry. Since it does not couple to quarks, it is difficult to produce at the LHC. The most promising option is to radiate a $Z_{L}$ from a pair of Drell-Yan produced leptons, in the process $p p \rightarrow \ell^{+} \ell^{-} Z_{L}$. The cross section for this process is calculated using the program CALCHEP [20] with the MRST2002 PDF set [104] and shown in Fig. 4.12, where one can see it is at most of order $10^{-2} \mathrm{fb}$ at the $14 \mathrm{TeV}$ LHC. As long as the new leptons are heavier than half the $Z_{L}$ mass, the gauge boson will decay into charged SM leptons with a branching ratio of 50\%, while the other $50 \%$ are into neutrinos (recalling there are three light $\nu_{R i}$ in this model). The final 


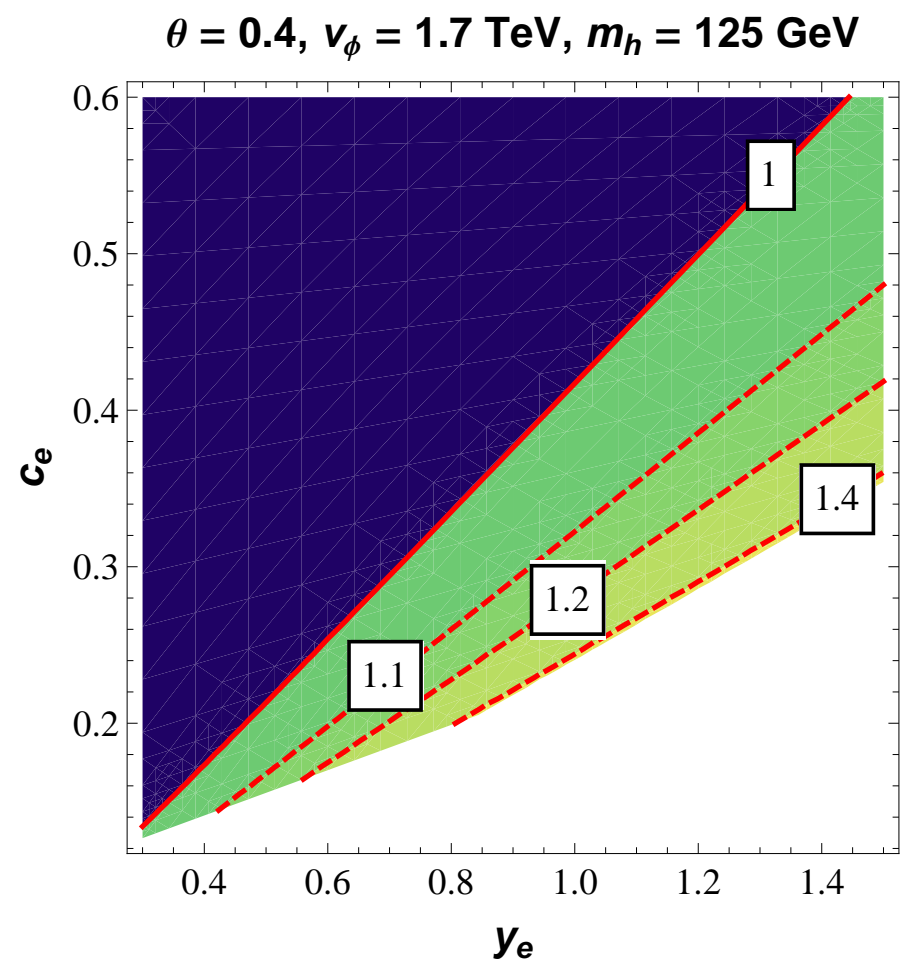

Figure 4.10. Contours of relative diphoton rate as a function of exotic charged lepton Yukawa couplings.

state with four charged leptons, two of which reconstruct the $Z_{L}$ mass, is essentially background free. Nevertheless even at a possible high luminosity upgrade of the LHC with $3 \mathrm{ab}^{-1}$ it will be difficult to probe $Z_{L}$ masses above $500 \mathrm{GeV}$.

Pairs of charged and neutal leptons can be pair produced at the LHC in the Drell-Yan process. The cross sections for the different processes at the $14 \mathrm{TeV}$ LHC are shown in Fig. 4.13 , and were again obtained using CALCHEP. The processes are similar to chargino/neutralino pair production, for which NLO corrections are moderate [105]. For this plot we have assumed that the lepton masses are given by Eq. (4.16) and Eq. 4.28). This leads to the following mass hierarchies for the exotic lepton sector,

$$
m_{e_{2}}>m_{\nu_{4}}>m_{e_{1}}>m_{\nu_{X}}
$$




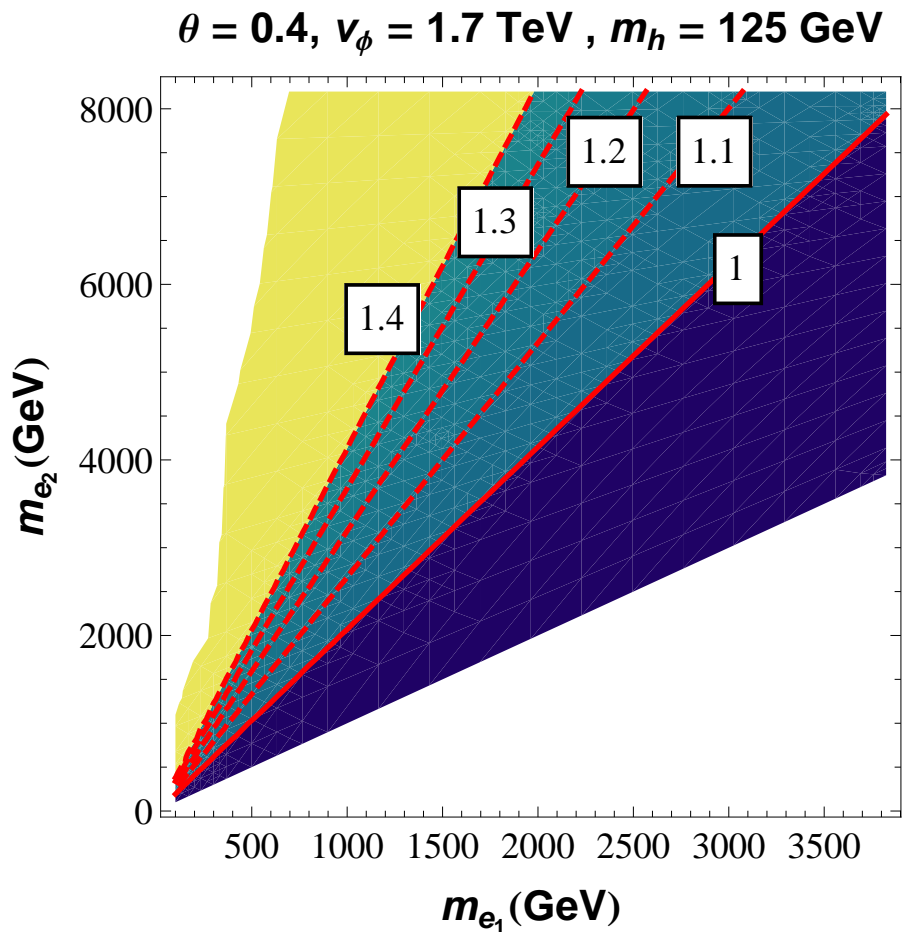

Figure 4.11. Contours of relative diphoton rate as a function of exotic charged lepton masses. Here we allow the masses to be as large as allowed by perturbativity and $v_{\phi}=1.7 \mathrm{TeV}$.

In this limit the mass splitting between $e_{1}$ and $e_{2}$ is given by $m_{e_{2}}-m_{e_{1}}=\sqrt{2} y_{e} v_{h}$ while $m_{\nu_{4}}-m_{e_{1}}=\frac{1}{\sqrt{2}} y_{e} v_{h}$. For $y_{e} \sim 0.8$ this gives a mass splitting of $\sim 280 \mathrm{GeV}$ between the charged leptons and a splitting of $\sim 140 \mathrm{GeV}$ between $e_{1}$ and $\nu_{4}$. Note also that for $y_{e} \sim 0.8$ and the $m_{e_{1}}$ range $100 \mathrm{GeV}-500 \mathrm{GeV}$ shown in Fig. 4.13 one also has $0.2 \lesssim c_{e} \lesssim 0.53$. The cross sections can be as large as one pb for particle masses close to the LEP limits, and up to $50 \mathrm{fb}$ for particle masses in the several hundred $\mathrm{GeV}$ range.

The decays of the exotic leptons will lead to a number of signatures at the LHC via their decays to electroweak gauge and Higgs bosons as well as DM. In the limits leading to Eq. 4.16. and Eq.(4.28) the heavy charged state $e_{2}$ can have the following decay chain,

$$
e_{2} \rightarrow W \nu_{4} \rightarrow W W e_{1} \rightarrow W W W \nu_{X} .
$$




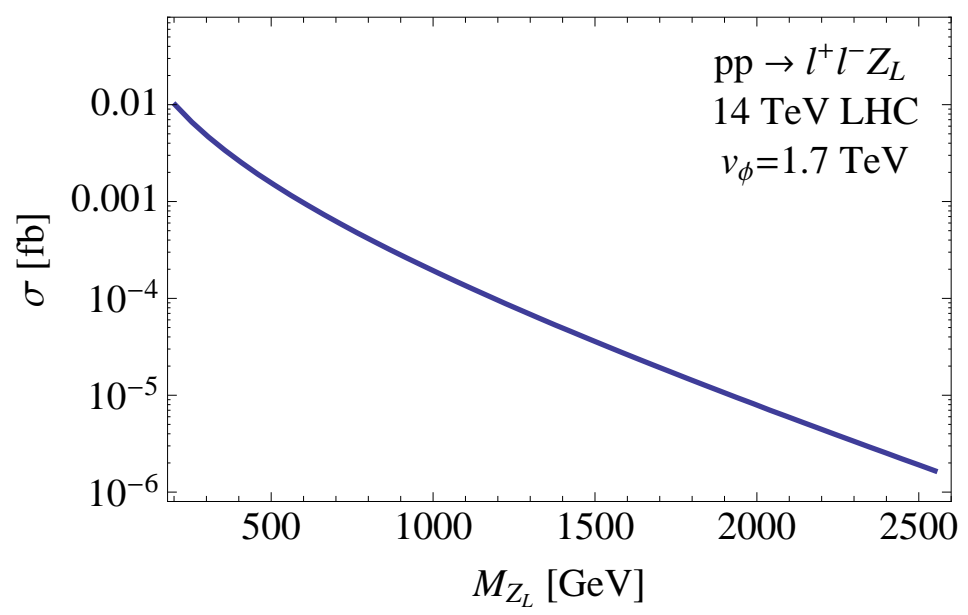

Figure 4.12. Cross section for the process $p p \rightarrow \ell^{+} \ell^{-} Z_{L}$ at the $14 \mathrm{TeV}$ LHC, for $v_{\phi}=1.7 \mathrm{TeV}$, and summed over SM leptons, $\ell^{ \pm}=e^{ \pm}, \mu^{ \pm}, \tau^{ \pm}$.

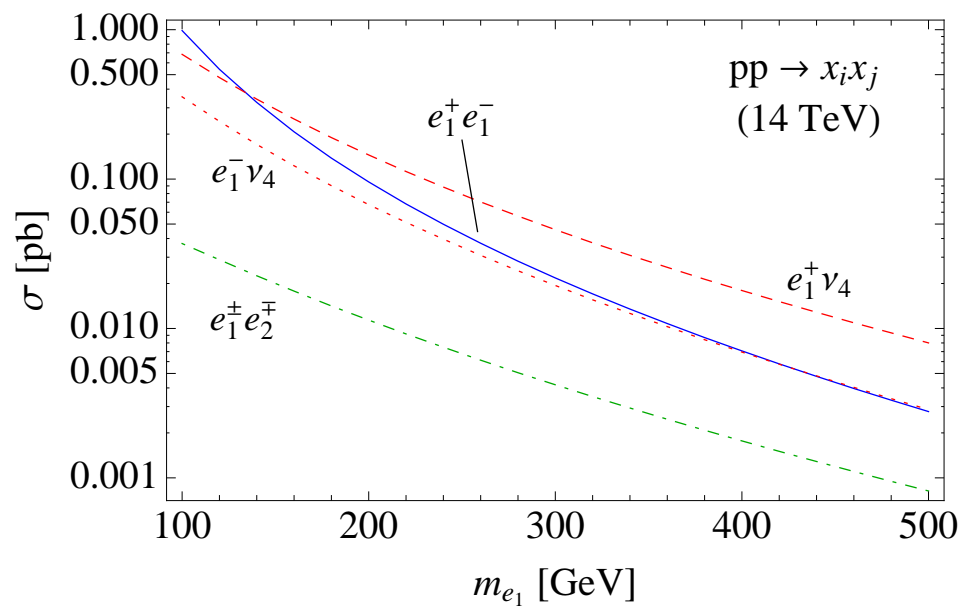

Figure 4.13. Cross sections for the pair production of exotic leptons at the $14 \mathrm{TeV}$ LHC, as a function of the lightest charged lepton mass $m_{e_{1}}$ in the limit leading to Eqs.4.16) and (4.28). For the processes involving $e_{2}^{ \pm}$and $\nu_{4}$ we have assumed that $m_{e_{2}}=m_{e_{1}}+280 \mathrm{GeV}$ which implies $m_{\nu_{4}}=$ $m_{e_{1}}+140 \mathrm{GeV}$.

Note that although we are neglecting mass mixing between $\nu_{X}$ and $\nu_{4}$ by assuming $y_{\nu} \ll 1$, it must be non-zero for the the heavy leptons to decay down to the DM. 
One can also have the heavy charged state decaying to DM more directly via,

$$
e_{2} \rightarrow W h \nu_{X}, e_{2} \rightarrow W Z \nu_{X}, e_{2} \rightarrow W \nu_{X}
$$

while the light charged state only has one tree level decay,

$$
e_{1} \rightarrow W \nu_{X}
$$

The heavy neutrino state $\nu_{4}$ can decay via $Z$ and $h$ bosons through,

$$
\nu_{4} \rightarrow Z \nu_{X}, \nu_{4} \rightarrow h \nu_{X}
$$

as well as $W$ bosons through,

$$
\nu_{4} \rightarrow W e_{1} \rightarrow W W \nu_{X}
$$

Thanks to the large mass differences between the particles, all intermediate gauge bosons are onshell, such that their final states can easily be reconstructed at the LHC. These decay patterns can change in more general lepton mixing scenarios, but should offer promising channels at the LHC.

For low masses, we see from Fig 4.13 that $e_{1}^{+} e_{1}^{-}$has the largest production rate. Assuming leptonic decays of the $W$-bosons, this leads to a signature

$$
p p \rightarrow e_{1}^{+} e_{1}^{-} \rightarrow W W E_{T} \rightarrow l^{+} l^{-} E_{T} .
$$

For larger masses the $e_{1}^{+} \nu_{4}$ channel becomes dominant, and can give rise to a striking trilepton signature through

$$
p p \rightarrow e_{1}^{+} \nu_{4} \rightarrow W Z E_{T} \rightarrow l^{+} l^{+} l^{-} \mathbb{E}_{T} .
$$


The signatures are similar to those from production of weakly charged supersymmetric particles at the LHC. While limits can be obtained in special cases from the $8 \mathrm{TeV}$ run of the LHC, we expect that at least $100 \mathrm{fb}^{-1}$ at the $14 \mathrm{TeV}$ LHC are needed to probe the exotic lepton sector at the LHC.

For light enough $\phi$ there is also the potential to produce it resonantly at the LHC through Higgs mixing. This scalar would inherit the SM Higgs decays, but be suppressed by $s_{\theta}^{2}$. Additionally, if kinematically allowed $\phi$ can also have the following decays to heavy leptons and dark matter,

$$
\begin{aligned}
& \phi \rightarrow e_{1} e_{1} \quad, \quad \phi \rightarrow e_{2} e_{2} \\
& \phi \rightarrow e_{1} e_{2} \quad, \quad \phi \rightarrow \nu_{4} \nu_{X}
\end{aligned}
$$

It can of course also decay to Higgs pairs $\phi \rightarrow h h$ when kinematically allowed. As discussed in Sec 4.2.5, however, for $v_{\phi} \sim 1.7 \mathrm{TeV}$ we typically have $\phi$ in the $\mathrm{TeV}$ range (see Fig 4.1) making it phenomenologically irrelevant for much of the parameter space.

\subsection{Conclusions/Outlook}

We have constructed a theory based on the gauging of lepton number, and found that for many choices of the parameters, the exotic leptons required to cancel gauge anomalies contain a dark matter candidate whose thermal relic density naturally saturates the requirements of cosmological observation. The dark matter is a Dirac (mostly singlet) neutrino and we find that masses $\gtrsim 200 \mathrm{GeV}$ give the correct thermal relic abundance via annihilation through the massive vector boson associated with the gauged lepton number. Higgs scalar mixing as well as 
gauge kinetic mixing which are found in this model also allow for a direct detection signal and give reasonably good prospects for detection in near future experiments.

The theory introduces only one new scale, the vacuum expectation value of a SM singlet scalar which breaks the lepton number and is constrained by experiment to be $\gtrsim 1.7 \mathrm{TeV}$. The global symmetry which stabilizes the dark matter is a consequence of the gauge structure and particle content of the the theory and does not need to be additionally imposed. Furthermore, as a consequence of the lepton number breaking, the dark matter is also accompanied by a set of vector like leptons charged under the SM gauge group with couplings to the SM Higgs. The same global symmetry which stabilizes the dark matter also prevents any dangerous flavor changing neutral currents or mass mixing with SM leptons. For a lepton breaking scale $\sim 1.7 \mathrm{TeV}$ phenomenologically viable dark matter and exotic vector-like leptons can be obtained.

The model contains a variety of potential LHC signals, though rates will be challenging. Some of the signatures, such as a four lepton final state with a $Z_{L}$ resonance in two of the leptons are fairly novel and specific, but otherwise most LHC phenomenology resembles other vector like lepton constructions along with singlet scalar phenomenology. The $14 \mathrm{TeV}$ run of the LHC should be able to probe some of the parameter space in the exotic lepton sector, although an $e^{+} e^{-}$collider with center of mass energies between $250 \mathrm{GeV}$ and $500 \mathrm{GeV}$ is more suitable for this task. Unless the $Z_{L}$ is very light, direct production is unlikely to be observable at the LHC. The indirect effect on four lepton interactions can however be probed at a linear collider, vastly extending the reach of the LEP experiments.

The exotic charged leptons can also lead to observable modifications of the Higgs decays and in particular to $h \rightarrow \gamma \gamma$, which is also affected by Higgs mixing. We have examined these effects for a range of model parameters and lepton masses which can potentially be produced at the LHC. Potential vacuum stability issues due to the presence of charged leptons with $\mathcal{O}(1)$ couplings to the Higgs can be alleviated with the presence of the gauge and scalar sector of this 
model, but one can also easily embed it into a more fundamental UV completion which would presumably solve such problems.

While $U(1)_{L}$ is an attractive gauge symmetry, which may contribute to the answer as to how dark matter can be massive and yet remain stable, many open questions remain in the current construction. For example, the hierarchy problem remains unaddressed, and almost certainly would require more structure and would lead to new phenomena. The current construction automatically contains new massive states as well as new interactions potentially containing $C P$-violating phases, which may be useful for explaining the baryon asymmetry of the Universe. One can also easily imagine embedding this model into a supersymmetric version or some other construction which solves the hierarchy problem or generates the lepton breaking scale naturally, but we leave these possibilities to a future study. 


\section{References}

[1] G. Aad et al. (ATLAS Collaboration), Phys.Lett. B716, 1 (2012), 1207.7214.

[2] S. Chatrchyan et al. (CMS Collaboration), Phys.Lett. B716, 30 (2012), 1207.7235.

[3] C. Quigg, pp. 3-67 (2002), hep-ph/0204104.

[4] S. Dawson (1994), hep-ph/9411325.

[5] J. S. Gainer, K. Kumar, I. Low, and R. Vega-Morales, JHEP 1111, 027 (2011), 1108.2274.

[6] D. Stolarski and R. Vega-Morales (2012), 1208.4840.

[7] Y. Chen, N. Tran, and R. Vega-Morales, JHEP 1301, 182 (2013), 1211.1959.

[8] P. Schwaller, T. M. P. Tait, and R. Vega-Morales (2013), 1305.1108.

[9] Y. Gao, A. V. Gritsan, Z. Guo, K. Melnikov, M. Schulze, et al., Phys.Rev. D81, 075022 (2010), 1001.3396.

[10] A. De Rujula, J. Lykken, M. Pierini, C. Rogan, and M. Spiropulu, Phys.Rev. D82, 013003 (2010), 1001.5300 .

[11] S. Bolognesi, Y. Gao, A. V. Gritsan, K. Melnikov, M. Schulze, et al. (2012), 1208.4018.

[12] R. Boughezal, T. J. LeCompte, and F. Petriello (2012), 1208.4311.

[13] J. Gunion and Z. Kunszt, Phys.Rev. D33, 665 (1986).

[14] A. Soni and R. Xu, Phys.Rev. D48, 5259 (1993), hep-ph/9301225.

[15] S. Choi, . Miller, D.J., M. Muhlleitner, and P. Zerwas, Phys.Lett. B553, 61 (2003), hep-ph/0210077.

[16] T. Matsuura and J. van der Bij, Z.Phys. C51, 259 (1991). 
[17] C. Buszello, I. Fleck, P. Marquard, and J. van der Bij, Eur.Phys.J. C32, 209 (2004), hep-ph/0212396.

[18] F. Fiedler, A. Grohsjean, P. Haefner, and P. Schieferdecker, Nucl.Instrum.Meth. A624, $203(2010), 1003.1316$.

[19] I. Volobouev (2011), 1101.2259.

[20] A. Belyaev, N. D. Christensen, and A. Pukhov (2012), 1207.6082.

[21] J. Alwall, M. Herquet, F. Maltoni, O. Mattelaer, and T. Stelzer, JHEP 1106, 128 (2011), 1106.0522 .

[22] P. Avery, D. Bourilkov, M. Chen, T. Cheng, A. Drozdetskiy, et al. (2012), 1210.0896.

[23] J. M. Campbell, W. T. Giele, and C. Williams (2012), 1205.3434.

[24] J. M. Campbell, W. T. Giele, and C. Williams (2012), 1204.4424.

[25] K. Hagiwara, R. Peccei, D. Zeppenfeld, and K. Hikasa, Nucl.Phys. B282, 253 (1987).

[26] Q.-H. Cao, C. Jackson, W.-Y. Keung, I. Low, and J. Shu, Phys.Rev. D81, 015010 (2010), 0911.3398.

[27] J. S. Gainer, W.-Y. Keung, I. Low, and P. Schwaller (2011), 1112.1405.

[28] B. Coleppa, K. Kumar, and H. E. Logan, Phys.Rev. D86, 075022 (2012), 1208.2692.

[29] I. Low, J. Lykken, and G. Shaughnessy (2012), 1207.1093.

[30] B. A. Dobrescu and J. D. Lykken, JHEP 1004, 083 (2010), 0912.3543.

[31] K. Nakamura et al. (Particle Data Group), J.Phys.G G37, 075021 (2010).

[32] N. D. Christensen and C. Duhr, Comput.Phys.Commun. 180, 1614 (2009), 0806.4194.

[33] S. Chatrchyan et al. (CMS Collaboration) (2012), 1210.3844.

[34] C. Zecher, T. Matsuura, and J. van der Bij, Z.Phys. C64, 219 (1994), hep-ph/9404295.

[35] T. Binoth, N. Kauer, and P. Mertsch, p. 142 (2008), 0807.0024.

[36] N. Kauer and G. Passarino, JHEP 1208, 116 (2012), 1206.4803.

[37] M. L. M. Jamin (1991). 
[38] T. Melia, P. Nason, R. Rontsch, and G. Zanderighi, JHEP 1111, 078 (2011), 1107.5051

[39] P. Nason, JHEP 0411, 040 (2004), hep-ph/0409146.

[40] S. Frixione, P. Nason, and C. Oleari, JHEP 0711, 070 (2007), 0709.2092.

[41] S. Alioli, P. Nason, C. Oleari, and E. Re, JHEP 1006, 043 (2010), 1002.2581.

[42] J. Pumplin, D. Stump, J. Huston, H. Lai, P. M. Nadolsky, et al., JHEP 0207, 012 (2002), hep-ph/0201195.

[43] V. Hankele, G. Klamke, D. Zeppenfeld, and T. Figy, Phys.Rev. D74, 095001 (2006), hep-ph/0609075.

[44] E. Gildener and S. Weinberg, Phys.Rev. D13, 3333 (1976).

[45] W. D. Goldberger, B. Grinstein, and W. Skiba, Phys.Rev.Lett. 100, 111802 (2008), 0708. 1463 .

[46] J. Fan, W. D. Goldberger, A. Ross, and W. Skiba, Phys.Rev. D79, 035017 (2009), 0803. 2040 .

[47] S. Dittmaier et al. (LHC Higgs Cross Section Working Group) (2011), long author list awaiting processing, 1101.0593 .

[48] M. Pivk and F. R. Le Diberder, Nucl.Instrum.Meth. A555, 356 (2005), physics/0402083.

[49] R. Cousins, J. Mumford, J. Tucker, and V. Valuev, JHEP 0511, 046 (2005).

[50] J. Beringer et al. (Particle Data Group), Phys.Rev. D86, 010001 (2012).

[51] R. Foot, G. C. Joshi, and H. Lew, Phys.Rev. D40, 2487 (1989).

[52] S. Rajpoot, Int.J.Theor.Phys. 27, 689 (1988).

[53] P. Fileviez Perez and M. B. Wise, Phys.Rev. D82, 011901 (2010), 1002.1754.

[54] T. R. Dulaney, P. Fileviez Perez, and M. B. Wise, Phys.Rev. D83, 023520 (2011), 1005. 0617 .

[55] P. Fileviez Perez and M. B. Wise, JHEP 1108, 068 (2011), 1106.0343.

[56] P. Fileviez Perez and M. B. Wise, Phys.Rev. D84, 055015 (2011), 1105.3190. 
[57] M. Duerr, P. Fileviez Perez, and M. B. Wise (2013), 1304.0576.

[58] W. Chao, Phys.Lett. B695, 157 (2011), 1005.1024.

[59] P. Dong and H. Long (2010), 1010.3818.

[60] A. Joglekar, P. Schwaller, and C. E. Wagner (2012), 1207.4235

[61] N. Arkani-Hamed, K. Blum, R. T. D’Agnolo, and J. Fan, JHEP 1301, 149 (2013), 1207. 4482

[62] B. Batell, S. Jung, and H. M. Lee (2012), 1211.2449.

[63] C. Arina, R. N. Mohapatra, and N. Sahu, Phys.Lett. B720, 130 (2013), 1211.0435.

[64] G. Moreau, Phys.Rev. D87, 015027 (2013), 1210.3977.

[65] W.-Z. Feng and P. Nath (2013), 1303.0289.

[66] A. Joglekar, P. Schwaller, and C. E. M. Wagner (2013), 1303.2969.

[67] H. Davoudiasl, I. Lewis, and E. Ponton (2012), 1211.3449.

[68] P. Fileviez Perez and M. B. Wise (2013), 1303.1452.

[69] K. Babu, C. F. Kolda, and J. March-Russell, Phys.Rev. D57, 6788 (1998), hep-ph/ 9710441

[70] K. R. Dienes, C. F. Kolda, and J. March-Russell, Nucl.Phys. B492, 104 (1997), hep-ph/ 9610479 .

[71] A. Hook, E. Izaguirre, and J. G. Wacker, Adv.High Energy Phys. 2011, 859762 (2011), 1006.0973 .

[72] J. Elias-Miro, J. R. Espinosa, G. F. Giudice, H. M. Lee, and A. Strumia, JHEP 1206, 031 (2012), 1203.0237

[73] M. Voloshin, Phys.Rev. D86, 093016 (2012), 1208.4303.

[74] D. McKeen, M. Pospelov, and A. Ritz, Phys.Rev. D86, 113004 (2012), 1208.4597.

[75] M. S. Carena, A. Daleo, B. A. Dobrescu, and T. M. Tait, Phys.Rev. D70, 093009 (2004), hep-ph/0408098. 
[76] E. Aprile et al. (XENON100 Collaboration), Phys.Rev.Lett. 109, 181301 (2012), 1207. 5988.

[77] P. Fileviez Perez, Phys.Lett. B711, 353 (2012), 1201.1501.

[78] B. Gripaios, A. Pomarol, F. Riva, and J. Serra, JHEP 0904, 070 (2009), 0902.1483.

[79] S. R. Coleman and E. J. Weinberg, Phys.Rev. D7, 1888 (1973).

[80] C. Englert, J. Jaeckel, V. V. Khoze, and M. Spannowsky (2013), 1301.4224.

[81] C. D. Carone and H. Murayama, Phys.Rev. D52, 484 (1995), hep-ph/9501220.

[82] R. Mohapatra, J.Phys. G36, 104006 (2009), 0902.0834.

[83] G. Servant and T. M. Tait, Nucl.Phys. B650, 391 (2003), hep-ph/0206071.

[84] P. Ade et al. (Planck Collaboration) (2013), 1303.5076.

[85] G. Belanger, F. Boudjema, P. Brun, A. Pukhov, S. Rosier-Lees, et al., Comput.Phys.Commun. 182, 842 (2011), 1004.1092.

[86] P. J. Fox, R. Harnik, J. Kopp, and Y. Tsai, Phys.Rev. D84, 014028 (2011), 1103.0240.

[87] D. Malling, D. Akerib, H. Araujo, X. Bai, S. Bedikian, et al. (2011), 1110.0103.

[88] O. Adriani et al. (PAMELA Collaboration), Nature 458, 607 (2009), 0810.4995.

[89] M. Ackermann et al. (Fermi LAT Collaboration), Phys.Rev.Lett. 108, 011103 (2012), 1109.0521.

[90] M. Aguilar et al. (AMS Collaboration), Phys.Rev.Lett. 110, 141102 (2013).

[91] O. Adriani et al. (PAMELA Collaboration), Phys.Rev.Lett. 105, 121101 (2010), 1007. 0821.

[92] M. Ackermann et al. (Fermi-LAT collaboration), Phys.Rev.Lett. 107, 241302 (2011), 1108.3546.

[93] C. Jackson, G. Servant, G. Shaughnessy, T. M. P. Tait, and M. Taoso (2013), 1302.1802.

[94] M. Ackermann et al. (LAT Collaboration), Phys.Rev. D86, 022002 (2012), 1205.2739.

[95] M. Aartsen et al. (IceCube collaboration), Phys.Rev.Lett. 110, 131302 (2013), 1212.4097. 
[96] M. Carena, I. Low, and C. E. Wagner, JHEP 1208, 060 (2012), 1206.1082.

[97] K. Kumar, R. Vega-Morales, and F. Yu, Phys.Rev. D86, 113002 (2012), 1205.4244.

[98] A. Falkowski, F. Riva, and A. Urbano (2013), 1303.1812.

[99] Tech. Rep. ATLAS-CONF-2013-034, CERN, Geneva (2013).

[100] Tech. Rep. CMS-PAS-HIG-13-005, CERN, Geneva (2013).

[101] A. Freitas and P. Schwaller, Phys.Rev. D87, 055014 (2013), 1211.1980.

[102] T. Appelquist and J. Carazzone, Phys.Rev. D11, 2856 (1975).

[103] B. Kyae and C. S. Shin (2013), 1303.6703.

[104] A. Martin, R. Roberts, W. Stirling, and R. Thorne, Eur.Phys.J. C28, 455 (2003), hep-ph/ 0211080 .

[105] W. Beenakker, R. Hopker, and M. Spira (1996), hep-ph/9611232. 


\section{APPENDIX A}

\section{Appendix}

\section{A.1. Angular Distributions}

In this Appendix we examine the various differential spectrums for the golden channel in more detail. The general scalar and background differential spectra are too cumbersome to write in one pag $\AA^{1}$ for most of the different components. We give a couple of the simplest ones here, but that are not found in literature. We also examine how the different signal hypotheses and background components contribute to the various kinematic distributions. We show a multitude of singly and doubly differential distributions for both signal and background. Of course none of these plots can show the discriminating power of the fully differential cross section, but one can visually get a sense for the discriminating power of these kinematic variables. Detector effects will also shape these distributions and deserve careful study, but it is clear that the golden channel is a powerful probe of the underlying physics.

\section{A.1.1. Analytic Expressions}

We give here a pair of analytic expressions for the differential mass spectra for one of the signal and one of the background components which are simple enough to fit on one page. Although not as powerful as using the fully differential cross section, with just these two relatively simple expressions one can perform robust analyses of the newly discovered scalar and its coupling to neutral gauge bosons as suggested in [12]. For the signal we give the $\varphi \rightarrow Z Z+Z \gamma \rightarrow 2 e 2 \mu$ differential mass spectrum including interference. For the $Z Z$ coupling we take only the 'SMlike' coupling $A_{1 Z Z}$ to be non-zero. For the $Z \gamma$ coupling we allow for both $A_{2 Z \gamma}$ and $A_{3 Z \gamma}$ to

${ }^{1}$ The distributions will be made public in the near future, but can be obtained from the corresponding author in the meantime. 
be non-zero, thus allowing for CP violation. Using Eq. 2.13 we obtain,

$$
\begin{aligned}
& \frac{d \Gamma_{S M+Z \gamma}}{d M_{1}^{2} d M_{2}^{2}}= \\
& \left(\sqrt { M _ { 1 } ^ { 4 } + ( M _ { 2 } ^ { 2 } - s ) ^ { 2 } - 2 M _ { 1 } ^ { 2 } ( M _ { 2 } ^ { 2 } + s ) } \left(6 A_{1 Z Z} A_{2 Z A} e_{l}\left(g_{L}+g_{R}\right)\left(g_{L}^{2}+g_{R}^{2}\right) M_{1} M_{2} m_{z}^{2}\left(2 M_{1}^{2} M_{2}^{2}-\left(M_{1}^{2}+M_{2}^{2}\right) m_{z}^{2}\right)\left(-1+\beta_{1}^{2}\right)\right.\right. \\
& \left(1+\beta_{1} \beta_{2}\right)\left(-1+\beta_{2}^{2}\right)+A_{1 Z Z}^{2}\left(g_{L}^{2}+g_{R}^{2}\right)^{2} M_{1}^{2} M_{2}^{2} m_{z}^{4} \sqrt{1-\beta_{1}^{2}} \sqrt{1-\beta_{2}^{2}}\left(3+2 \beta_{1} \beta_{2}-2 \beta_{2}^{2}+\beta_{1}^{2}\left(-2+3 \beta_{2}^{2}\right)\right)+2 e_{l}^{2} \sqrt{1-\beta_{1}^{2}} \sqrt{1-\beta_{2}^{2}} \\
& \left(2 A_{3 Z A}^{2}\left(\beta_{1}+\beta_{2}\right)^{2}+A_{2 Z A}^{2}\left(3-\beta_{1}^{2}+4 \beta_{1} \beta_{2}+\left(-1+3 \beta_{1}^{2}\right) \beta_{2}^{2}\right)\right)\left(2 g_{L} g_{R} M_{1}^{2} M_{2}^{2}\left(\left(M_{1}-m_{z}\right)\left(M_{2}-m_{z}\right)\left(M_{1}+m_{z}\right)\left(M_{2}+m_{z}\right)+m_{z}^{2} \Gamma_{z}^{2}\right)\right. \\
& +g_{L}^{2}\left(M_{2}^{4} m_{z}^{2}\left(m_{z}^{2}+\Gamma_{z}^{2}\right)+M_{1}^{2} M_{2}^{2} m_{z}^{2}\left(-3 M_{2}^{2}+m_{z}^{2}+\Gamma_{z}^{2}\right)+M_{1}^{4}\left(3 M_{2}^{4}-3 M_{2}^{2} m_{z}^{2}+m_{z}^{4}+m_{z}^{2} \Gamma_{z}^{2}\right)\right)+g_{R}^{2}\left(M_{2}^{4} m_{z}^{2}\left(m_{z}^{2}+\Gamma_{z}^{2}\right)\right. \\
& \left.\left.\left.\left.+M_{1}^{2} M_{2}^{2} m_{z}^{2}\left(-3 M_{2}^{2}+m_{z}^{2}+\Gamma_{z}^{2}\right)+M_{1}^{4}\left(3 M_{2}^{4}-3 M_{2}^{2} m_{z}^{2}+m_{z}^{4}+m_{z}^{2} \Gamma_{z}^{2}\right)\right)\right)\right)\right) \\
& /\left(4608 \pi^{4} s^{2} v_{h}^{2}\left(1-\beta_{1}^{2}\right)^{3 / 2}\left(1-\beta_{2}^{2}\right)^{3 / 2}\left(\left(M_{1}^{2}-m_{z}^{2}\right)^{2}+m_{z}^{2} \Gamma_{z}^{2}\right)\left(\left(M_{2}^{2}-m_{z}^{2}\right)^{2}+m_{z}^{2} \Gamma_{z}^{2}\right)\right)
\end{aligned}
$$

where we define,

$$
\beta_{1,2}=\sqrt{1-\frac{4 M_{1}^{2}}{\left(1 \pm\left(M_{1}^{2}-M_{2}^{2}\right) / s\right)^{2} s}} .
$$

This expression is frame invariant and can accommodate a Higgs-like particle with SM couplings to $Z Z$, but with perhaps new physics contributions through its couplings to $Z \gamma$. The $e_{l}$ are the photon couplings to charged leptons while $g_{L, R}$ are the leptonic $Z$ couplings. $M_{1}$ and $M_{2}$ are the final state lepton pair invariant masses while $m_{Z}$ is the mass of the $Z$ boson and $\sqrt{s}$ is the four lepton system invariant mass. The doubly differential mass spectrum for the full $t+u$ component of the background (sum of components $B$ - $E$ ) can 
be obtained analytically via Eq. 2.30 to give,

$$
\begin{aligned}
& \frac{d \sigma_{t+u}^{B G}}{d M_{1}^{2} d M_{2}^{2}}= \\
& -\left(\left(\left(g_{q L}^{4}+g_{q R}^{4}\right)\left(g_{L}^{2}+g_{R}^{2}\right)^{2} M_{1}^{4} M_{2}^{4}+2 e_{l} e_{q}\left(g_{q L}^{3}+g_{q R}^{3}\right)\left(g_{L}+g_{R}\right)\left(g_{L}^{2}+g_{R}^{2}\right) M_{1}^{2} M_{2}^{2}\left(2 M_{1}^{2} M_{2}^{2}-\left(M_{1}^{2}+M_{2}^{2}\right) m_{z}^{2}\right)\right.\right. \\
& +8 e_{l}^{4} e_{q}^{4}\left(\left(M_{1}^{2}-m_{z}^{2}\right)^{2}+m_{z}^{2} \Gamma_{z}^{2}\right)\left(\left(M_{2}^{2}-m_{z}^{2}\right)^{2}+m_{z}^{2} \Gamma_{z}^{2}\right)+4 e_{l}^{3} e_{q}^{3}\left(g_{q L}+g_{q R}\right)\left(g_{L}+g_{R}\right)\left(\left(M_{2}-m_{z}\right)\left(M_{2}+m_{z}\right)\left(-M_{1}^{2}+m_{z}^{2}\right)\right. \\
& \left.\left(-2 M_{1}^{2} M_{2}^{2}+\left(M_{1}^{2}+M_{2}^{2}\right) m_{z}^{2}\right)+m_{z}^{2}\left(M_{1}^{4}+M_{2}^{4}-\left(M_{1}^{2}+M_{2}^{2}\right) m_{z}^{2}\right) \Gamma_{z}^{2}\right)+2 e_{l}^{2} e_{q}^{2}\left(g_{q L}^{2}+g_{q R}^{2}\right)\left(4 g_{L} g_{R} M_{1}^{2} M_{2}^{2}\left(M_{1}-m_{z}\right)\left(M_{2}-m_{z}\right)\right. \\
& \left(M_{1}+m_{z}\right)\left(M_{2}+m_{z}\right)+g_{L}^{2}\left(\left(-2 M_{1}^{2} M_{2}^{2}+\left(M_{1}^{2}+M_{2}^{2}\right) m_{z}^{2}\right)^{2}+\left(M_{1}^{4}+M_{2}^{4}\right) m_{z}^{2} \Gamma_{z}^{2}\right)+g_{R}^{2}\left(\left(-2 M_{1}^{2} M_{2}^{2}+\left(M_{1}^{2}+M_{2}^{2}\right)\right.\right. \\
& \left.\left.\left.\left.m_{z}^{2}\right)^{2}+\left(M_{1}^{4}+M_{2}^{4}\right) m_{z}^{2} \Gamma_{z}^{2}\right)\right)\right)\left(4\left(M_{1}^{2}+M_{2}^{2}-s\right) \sqrt{M_{1}^{4}+\left(M_{2}^{2}-s\right)^{2}-2 M_{1}^{2}\left(M_{2}^{2}+s\right)}-\left(\left(M_{1}^{2}+M_{2}^{2}\right)^{2}+s^{2}\right)\right. \\
& \left.\left.\left(\log \left[\left(M_{1}^{2}+M_{2}^{2}-s+\sqrt{M_{1}^{4}+\left(M_{2}^{2}-s\right)^{2}-2 M_{1}^{2}\left(M_{2}^{2}+s\right)}\right)^{2}\right]-2 \log \left[-M_{1}^{2}-M_{2}^{2}+s+\sqrt{M_{1}^{4}+\left(M_{2}^{2}-s\right)^{2}-2 M_{1}^{2}\left(M_{2}^{2}+s\right)}\right]\right)\right)\right) \\
& /\left(27648 M_{1}^{2} M_{2}^{2} \pi^{5}\left(M_{1}^{2}+M_{2}^{2}-s\right) s^{2}\left(\left(M_{1}^{2}-m_{z}^{2}\right)^{2}+m_{z}^{2} \Gamma_{z}^{2}\right)\left(\left(M_{2}^{2}-m_{z}^{2}\right)^{2}+m_{z}^{2} \Gamma_{z}^{2}\right)\right) .
\end{aligned}
$$

This expression includes the $Z Z, Z \gamma$ and $\gamma \gamma$ contributions including all interference and can be combined with $p d f s$ or be used for a leptonic initial state. The $e_{q}$ are the photon couplings to the initial state fermions while the $g_{q R / L}$ are the initial state fermion couplings to $Z$ bosons. Note that these expressions have not been normalized and should be thought of as at fixed $s$.

\section{A.1.2. Singly Differential Angular Distributions}

In Fig. A.1 A.5 we show the angular distributions for the 5 angles $\left(\cos \Theta, \cos \theta_{1}, \cos \theta_{2}, \Phi_{1}, \Phi\right)$ found in the four lepton system and defined in Sec. 3.1. We plot the angular distributions for signal hypotheses 1-5 defined in Sec. 2.3.2 and also show the various background components defined in Sec. 2.4.2. For all distributions the phase space is defined as $4 \mathrm{GeV}$ $<M_{1}<120 \mathrm{GeV}$ and $4 \mathrm{GeV}<M_{2}<120 \mathrm{GeV}$ with $\sqrt{\hat{s}}=125 \mathrm{GeV}$ for signal and $110 \mathrm{GeV}<\sqrt{\hat{s}}<140 \mathrm{GeV}$ for background. 
Since we are considering a spin- 0 scalar as our signal, the $\cos \Theta$ and $\Phi_{1}$ are of course flat, but are still useful for discriminating between signal and background. A particularly interesting variable is the azimuthal angle between the lepton decay planes, $\Phi$. This is especially sensitive to the various interference effects as well as the CP properties of the decaying scalar, as was pointed out in [26]. One can see that the different signal hypothesis affect the modulation of $\Phi$ while an extreme case like the CP violating hypothesis 5 can lead to a striking signal in the form of an asymmetric modulation and phase shift relative to the SM prediction.

For the background we can see how the various components contribute to the different kinematic variables. It is clear that the $Z \gamma t+u$ component $(D)$ is the dominant contribution for our defined phase space. Note however, that the s-channel component $(A)$ also contributes and in particular is the dominant contribution to the modulation of $\Phi$. We can also see that the resonant process affects $\cos \theta_{1}$ and $\cos \theta_{2}$, especially in the forward regions. It is also interesting to comment that the $\gamma \gamma$ contribution $(B)$ is featureless in all of the distributions except for a small upward slope in the extreme forward directions of $\cos \Theta$. Note that for the $\Phi_{1}$ azimuthal angle, the modulation is due entirely to the $Z \gamma t+u$ component $(D)$. Whether these different effects can still be seen once detector effects are included requires careful study which we leave for future work.

\section{A.1.3. Doubly Differential spectra}

In Fig. A.6, A.12 we show various combinations of the doubly differential spectra for the five signal hypotheses as well as the full background. These are primarily for illustration purposes, but from these one can get an idea of the correlations between the different 

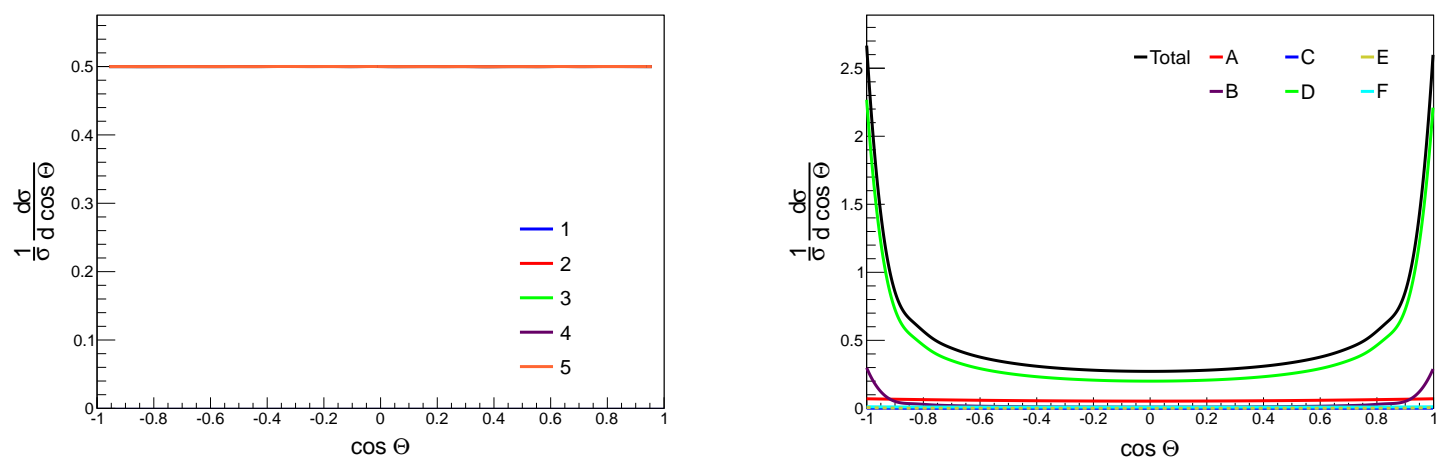

Figure A.1. On the left hand side we have plotted the $\cos \Theta$ angular distributions for hypotheses $1-5$ (hypothesis $1 \equiv \mathrm{SM}$ ) defined in Sec 2.3.2. On the right hand side we plot the components A-F of the background defined in Sec.2.4.2.
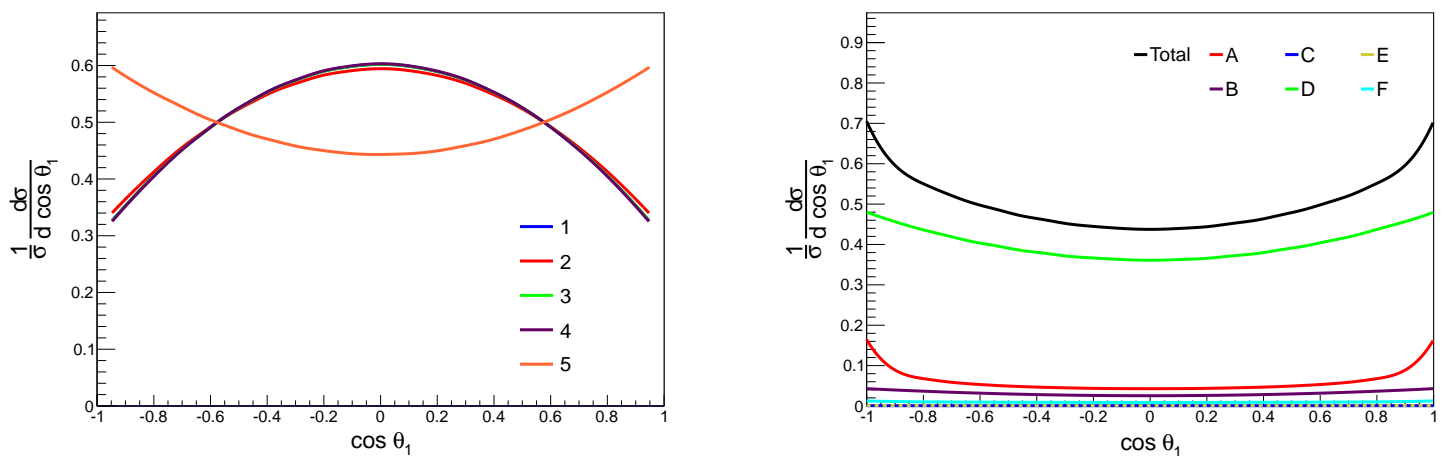

Figure A.2. On the left hand side we have plotted the $\cos \theta_{1}$ angular distributions for hypotheses $1-5$ (hypothesis $1 \equiv \mathrm{SM}$ ) defined in Sec 2.3.2. On the right hand side we plot the components A-F of the background defined in Sec.2.4.2,

kinematic variables ${ }^{2}$. For these plots only the five signal hypotheses and the full result for the background are shown. For all distributions the phase space is defined as $4 \mathrm{GeV}$ $<M_{1}<120 \mathrm{GeV}$ and $4 \mathrm{GeV}<M_{2}<120 \mathrm{GeV}$ with $\sqrt{\hat{s}}=125 \mathrm{GeV}$ for signal and $110 \mathrm{GeV}<\sqrt{\hat{s}}<140 \mathrm{GeV}$ for background.

\footnotetext{
${ }^{2}$ We do not show all possible combinations, but any not shown here can be obtained by emailing the corresponding author
} 

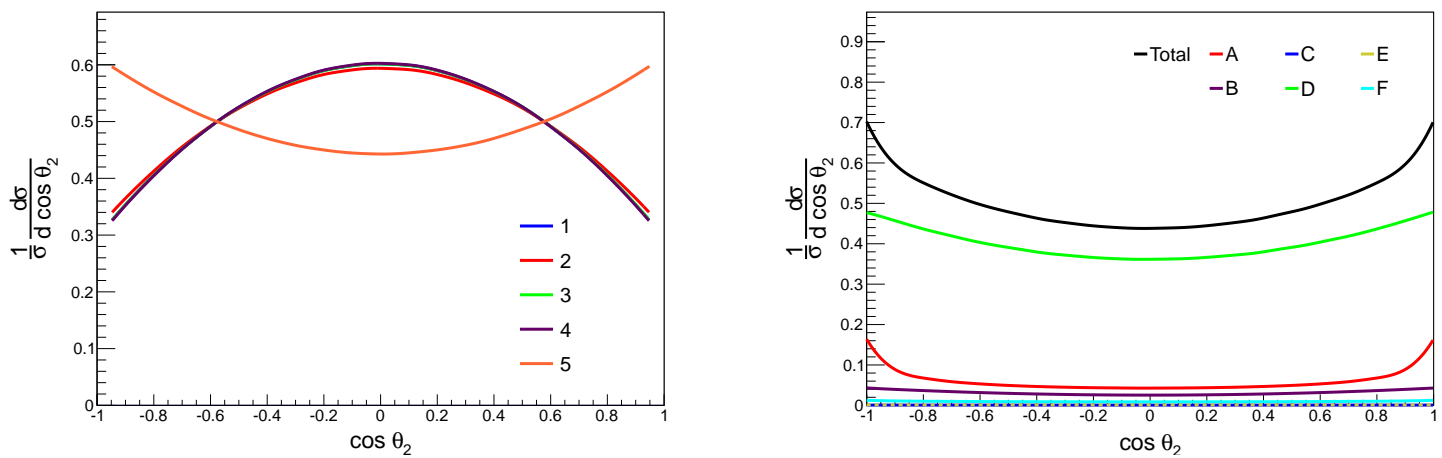

Figure A.3. On the left hand side we have plotted the $\cos \theta_{2}$ angular distributions for hypotheses 1-5 (hypothesis $1 \equiv \mathrm{SM}$ ) defined in Sec 2.3.2. On the right hand side we plot the components A-F of the background defined in Sec.2.4.2.
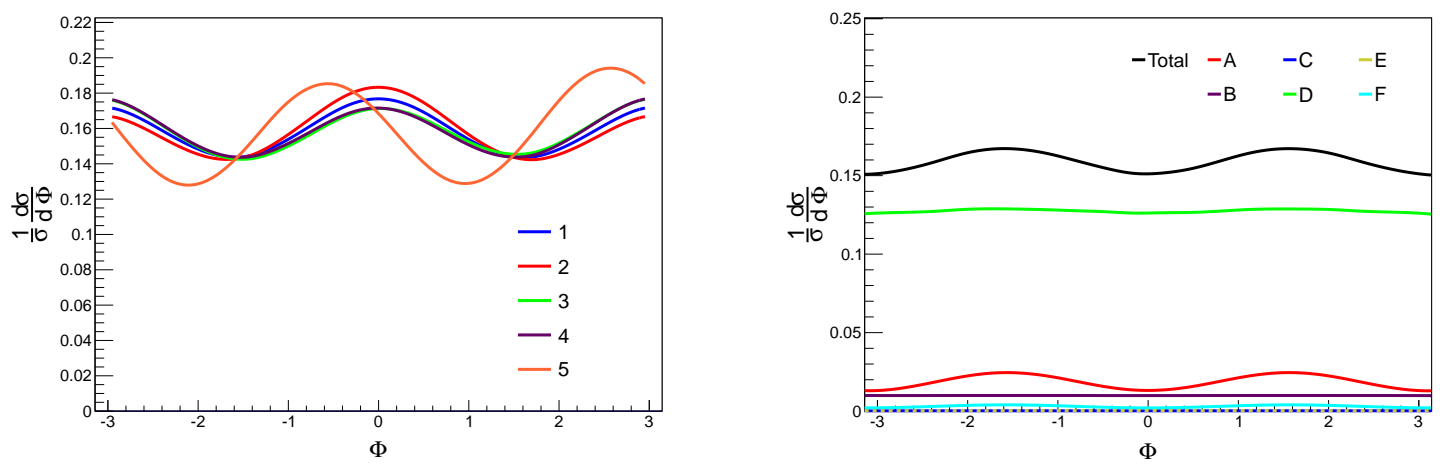

Figure A.4. On the left hand side we have plotted the $\Phi$ angular distributions for hypotheses 1-5 (hypothesis $1 \equiv \mathrm{SM}$ ) defined in Sec 2.3.2. On the right hand side we plot the components A-F of the background defined in Sec.2.4.2. 

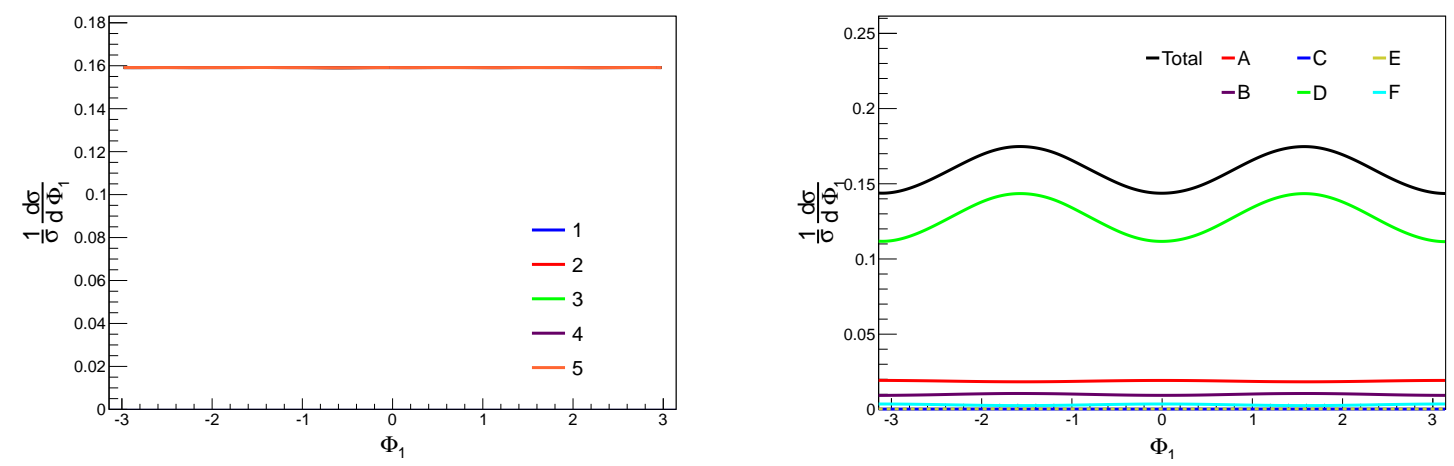

Figure A.5. On the left hand side we have plotted the $\Phi_{1}$ angular distributions for hypotheses 1-5 (hypothesis $1 \equiv \mathrm{SM}$ ) defined in Sec 2.3.2. On the right hand side we plot the components A-F of the background defined in Sec.2.4.2.
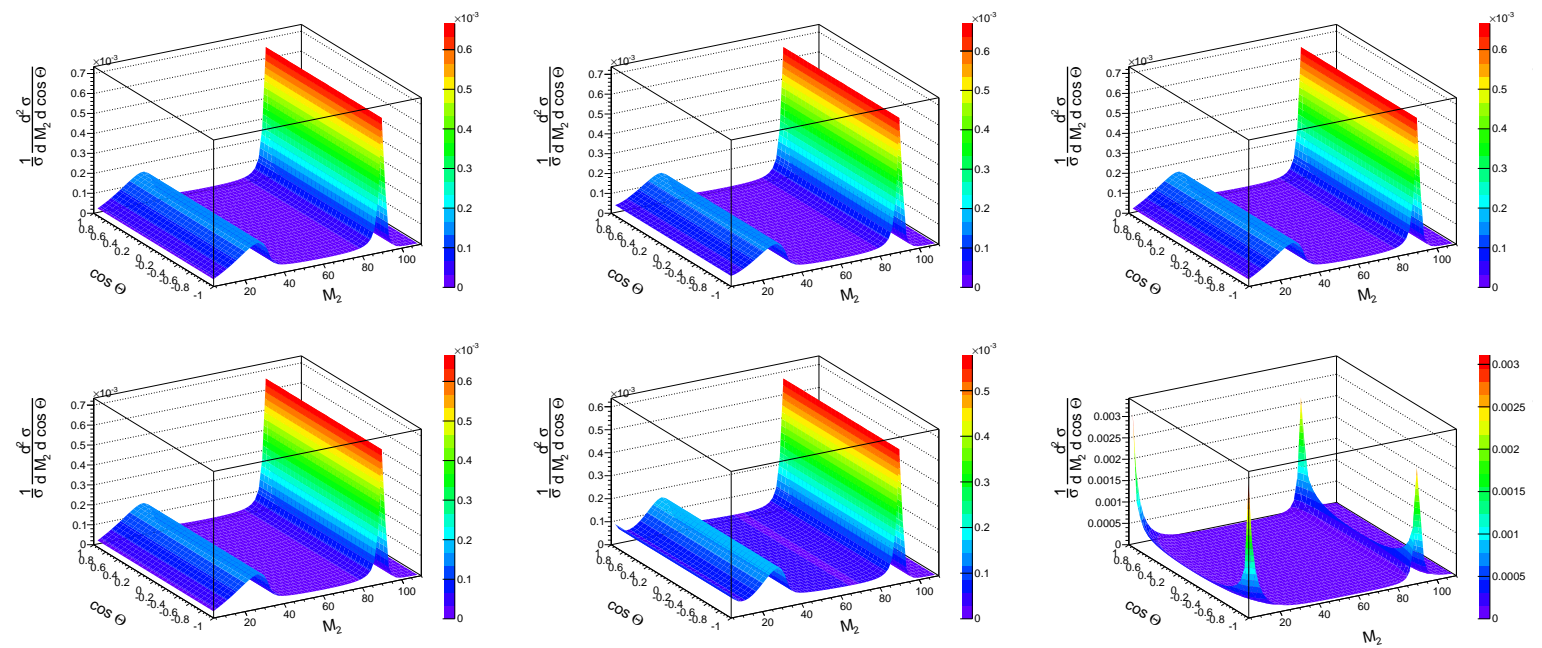

Figure A.6. The $\left(M_{2}, \cos \Theta\right)$ doubly differential spectrum. The first five distributions are for signal hypotheses $1-5$ (hypothesis $1 \equiv \mathrm{SM}$ in top left) defined in Sec.2.3.2 while the bottom right plot is for the full background. 

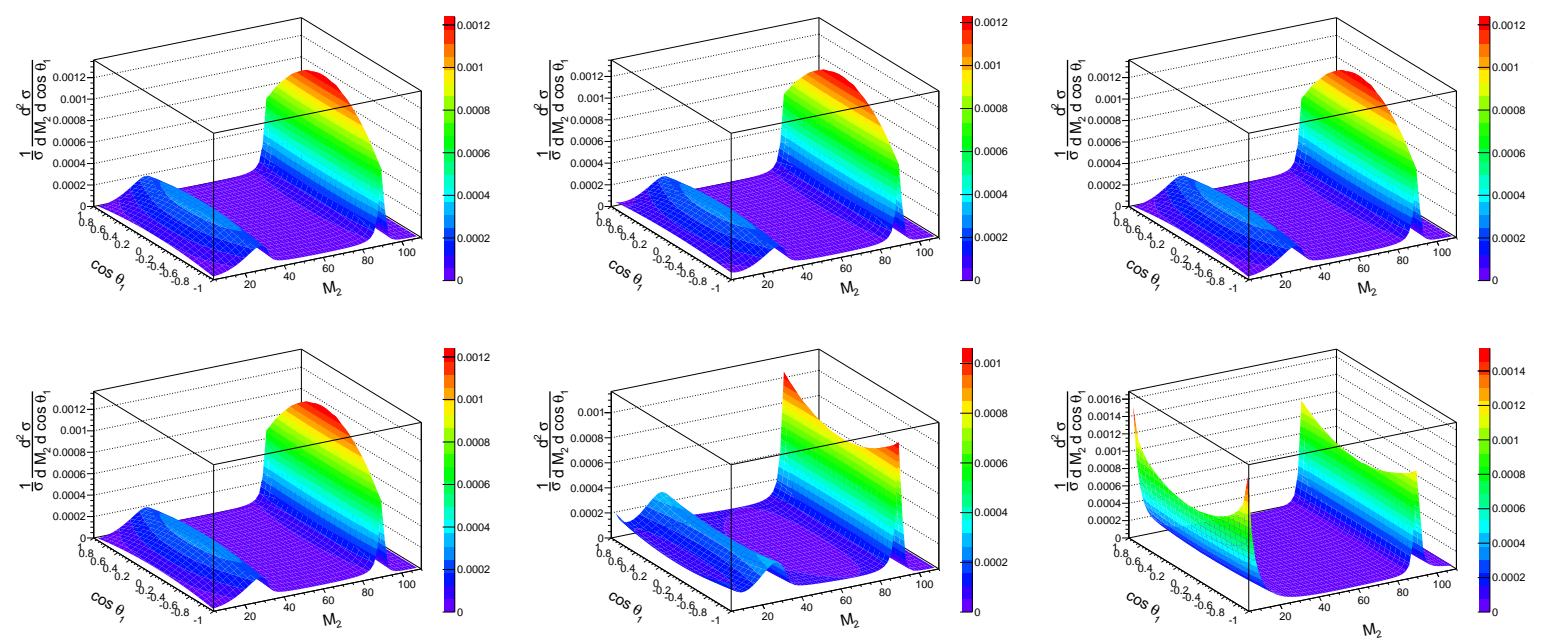

Figure A.7. The $\left(M_{2}, \cos \theta_{1}\right)$ doubly differential spectrum. The first five distributions are for signal hypotheses $1-5$ (hypothesis $1 \equiv \mathrm{SM}$ in top left) defined in Sec.2.3.2 while the bottom right plot is for the full background.
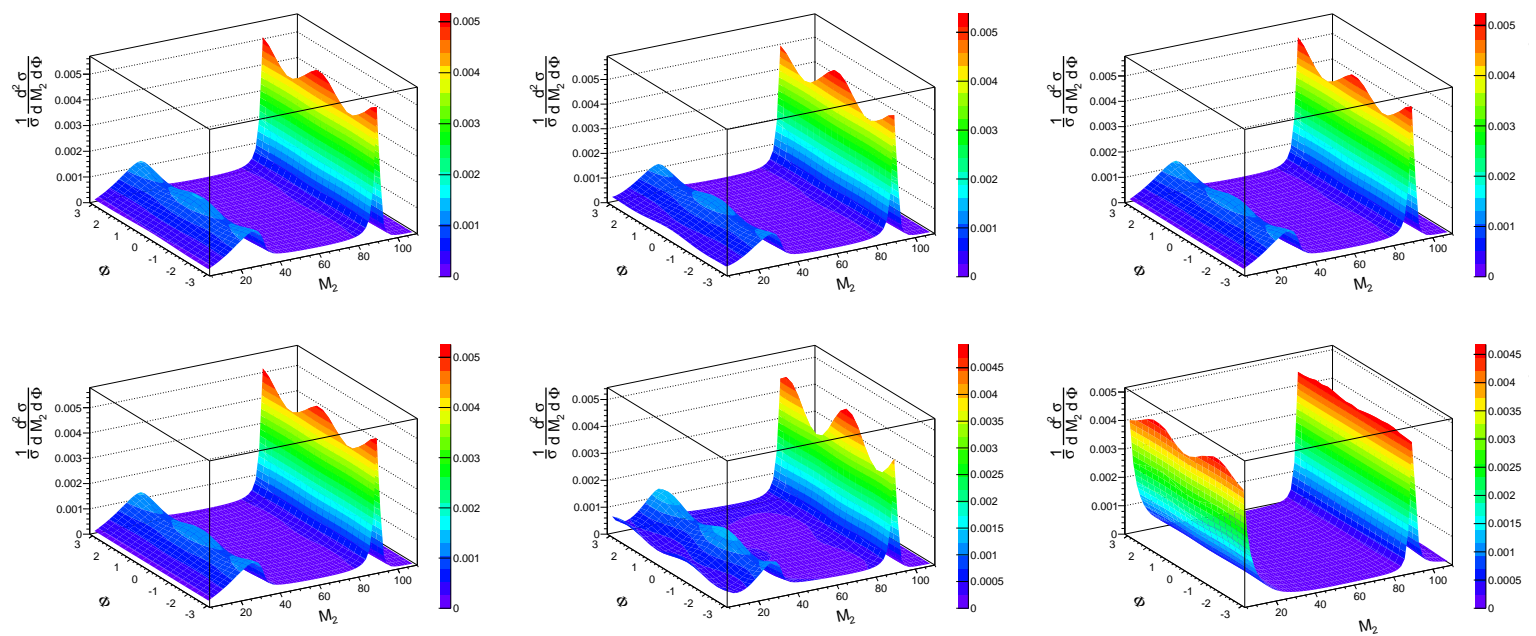

Figure A.8. The $\left(M_{2}, \Phi\right)$ doubly differential spectrum. The first five distributions are for signal hypotheses 1-5 (hypothesis $1 \equiv \mathrm{SM}$ in top left) defined in Sec.2.3.2 while the bottom right plot is for the full background. 

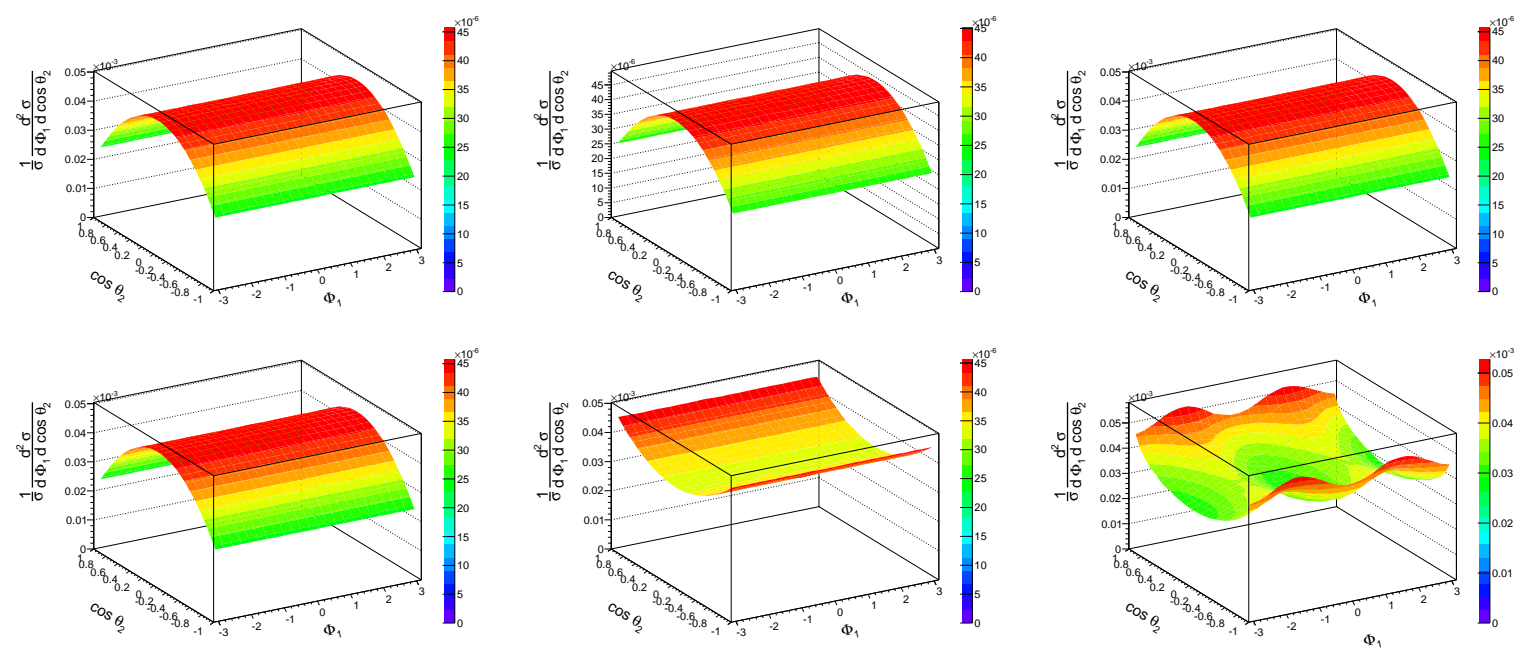

Figure A.9. The $\left(\cos \theta_{2}, \Phi_{1}\right)$ doubly differential spectrum. The first five distributions are for signal hypotheses $1-5$ (hypothesis $1 \equiv \mathrm{SM}$ in top left) defined in Sec.2.3.2 while the bottom right plot is for the full background.
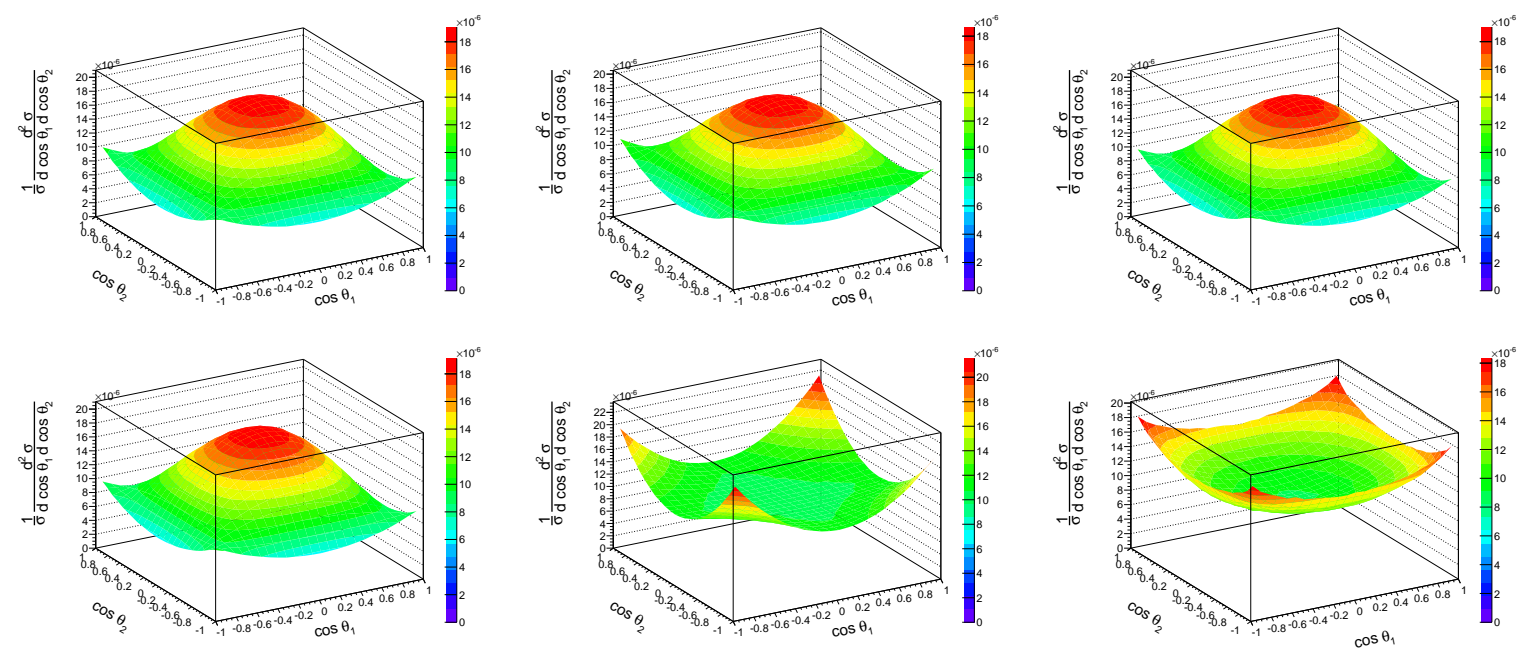

Figure A.10. The $\left(\cos \theta_{1}, \cos \theta_{2}\right)$ doubly differential spectrum. The first five distributions are for signal hypotheses $1-5$ (hypothesis $1 \equiv \mathrm{SM}$ in top left) defined in Sec.3.2 while the bottom right plot is for the full background. 

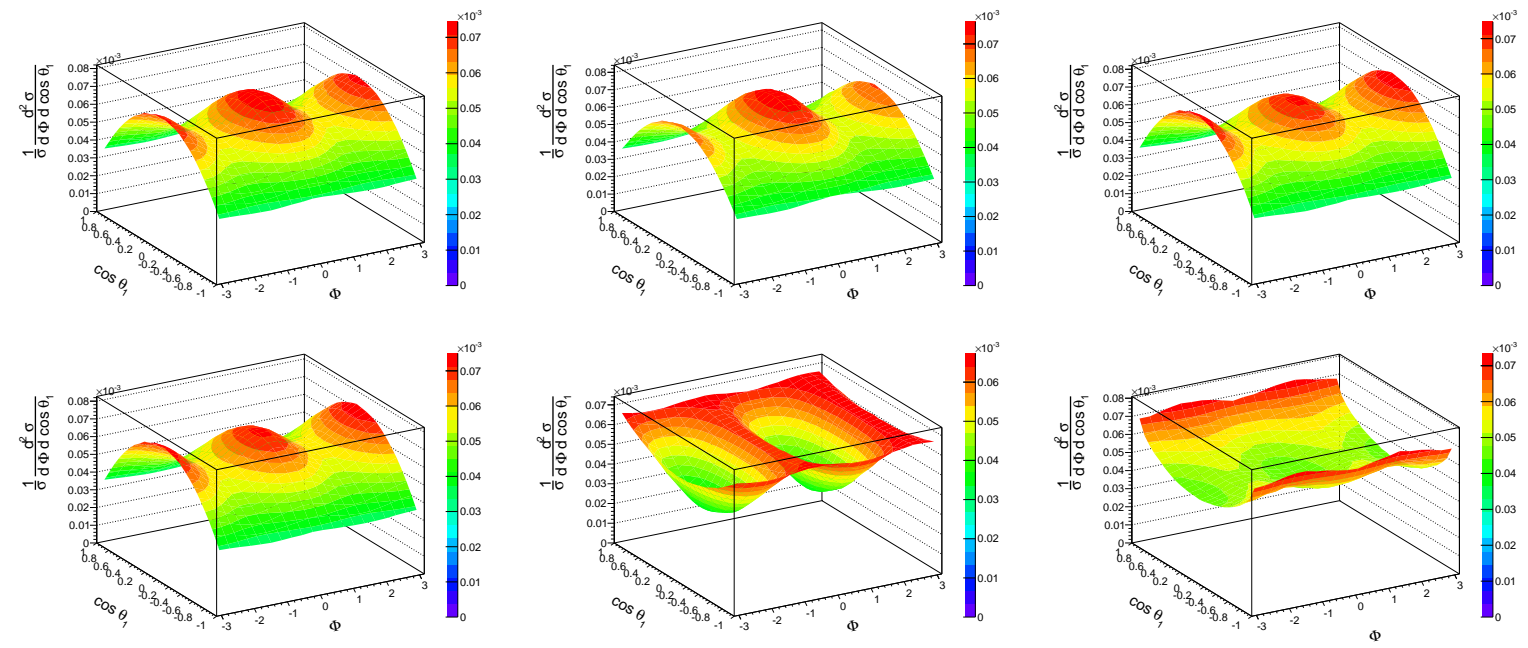

Figure A.11. The $\left(\Phi, \cos \theta_{1}\right)$ doubly differential spectrum. The first five distributions are for signal hypotheses $1-5$ (hypothesis $1 \equiv$ SM in top left) defined in Sec.2.3.2 while the bottom right plot is for the full background.
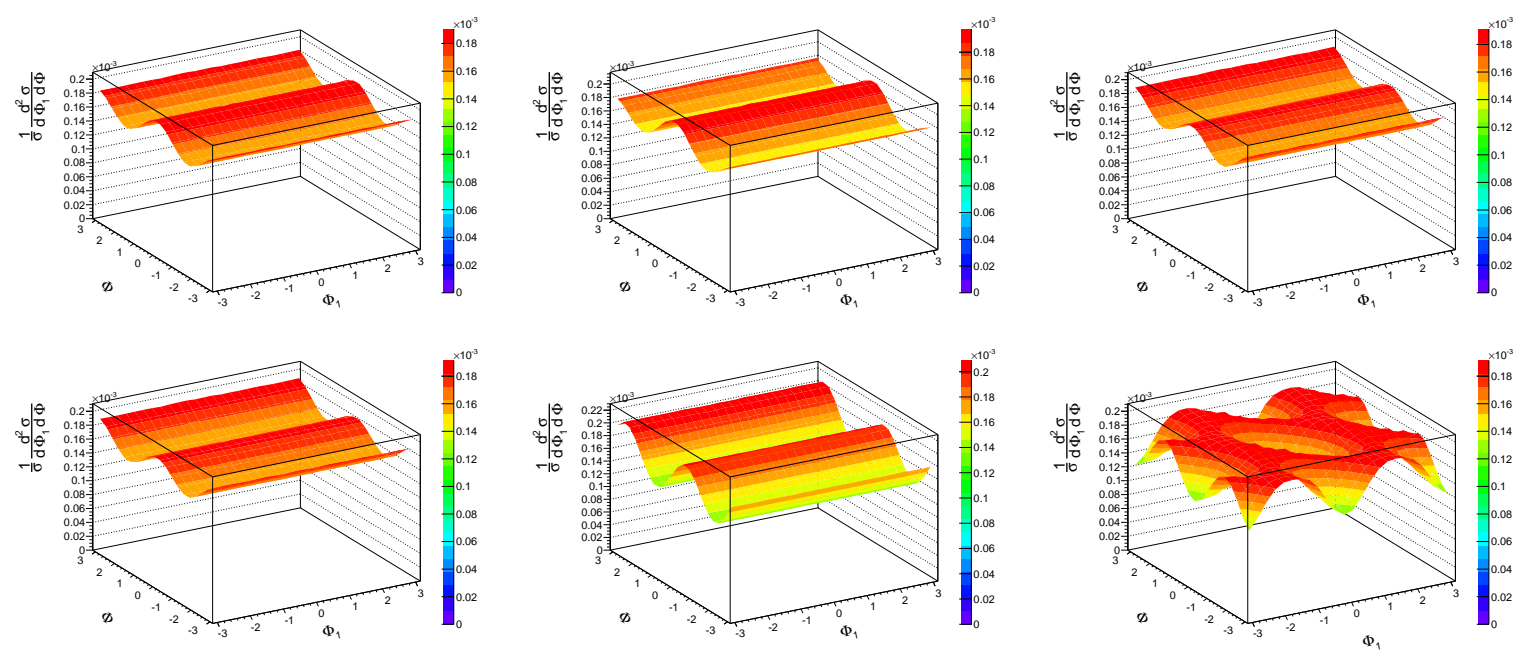

Figure A.12. The $\left(\Phi, \Phi_{1}\right)$ doubly differential spectrum. The first five distributions are for signal hypotheses $1-5$ (hypothesis $1 \equiv \mathrm{SM}$ in top left) defined in Sec.3.2 while the bottom right plot is for the full background. 